\title{
Cooperative diversity for the cellular uplink: Sharing strategies, performance analysis, and receiver design
}

\author{
Kanchan G. Vardhe \\ West Virginia University
}

Follow this and additional works at: https://researchrepository.wvu.edu/etd

\section{Recommended Citation}

Vardhe, Kanchan G., "Cooperative diversity for the cellular uplink: Sharing strategies, performance analysis, and receiver design" (2005). Graduate Theses, Dissertations, and Problem Reports. 3221. https://researchrepository.wvu.edu/etd/3221

This Thesis is protected by copyright and/or related rights. It has been brought to you by the The Research Repository @ WVU with permission from the rights-holder(s). You are free to use this Thesis in any way that is permitted by the copyright and related rights legislation that applies to your use. For other uses you must obtain permission from the rights-holder(s) directly, unless additional rights are indicated by a Creative Commons license in the record and/ or on the work itself. This Thesis has been accepted for inclusion in WVU Graduate Theses, Dissertations, and Problem Reports collection by an authorized administrator of The Research Repository @ WVU. For more information, please contact researchrepository@mail.wvu.edu. 


\title{
Cooperative Diversity for the Cellular Uplink: Sharing Strategies, Performance Analysis, and Receiver Design
}

\author{
by \\ Kanchan G. Vardhe \\ Thesis submitted to the \\ College of Engineering and Mineral Resources \\ at West Virginia University \\ in partial fulfillment of the requirements \\ for the degree of \\ Master of Science \\ in \\ Electrical Engineering \\ Daryl Reynolds, Ph.D., Chair \\ Matthew C.Valenti, Ph.D. \\ Arun Ross, Ph.D.
}

Lane Department of Computer Science and Electrical Engineering

Morgantown, West Virginia

2005

Keywords: Cooperative Diversity, MIMO, Decode and Forward, Space Time Codes, Information Outage Probability

Copyright 2005 Kanchan G. Vardhe 


\author{
Abstract \\ Cooperative Diversity for the Cellular Uplink: Sharing Strategies, Performance Analysis, \\ and Receiver Design \\ by \\ Kanchan G. Vardhe \\ Master of Science in Electrical Engineering \\ West Virginia University \\ Daryl Reynolds, Ph.D., Chair
}

High voice quality, high data rate, and low complexity at the remote mobile units are the demanding requirements of the next generation wireless communications. Multipath fading channel degrades the performance of wireless communications and hence places limits on these requirements. It is a very challenging task to effectively combat or reduce the effect of fading without increasing the transmit power or the complexity at the mobile units. In such fading environments, antenna diversity serves as an attractive solution. But the use of multiple antennas to achieve transmit diversity in the uplink cellular system is impractical due to size and power constraints at the mobile users. A new form of spatial diversity called 'cooperative diversity' has been proposed which eliminates the need for incorporating multiple antennas on a single mobile unit, while still providing diversity gains via cooperation of distributed users in the system.

In this thesis, we propose data sharing schemes for the cooperative diversity in a cellular uplink to exploit diversity and enhance throughput performance of the system. Particularly, we consider new two and three-or-more user decode and forward (DF) protocols using space time block codes. We discuss two-user and three-user amplify and forward (AF) protocols and evaluate the performance of the above mentioned data sharing protocols in terms of the bit error rate and the throughput in an asynchronous code division multiple access (CDMA) cellular uplink. We develop a linear receiver for joint space-time decoding and multiuser detection that provides full diversity and near maximum-likelihood performance.

We also focus on a practical situation where inter-user channel is noisy and cooperating users can not successfully estimate other user's data. We further design our system model such that, users decide not to forward anything in case of symbol errors. Channel estimation plays an important role here, since cooperating users make random estimation errors and the base station can not have the knowledge of the errors or the inter-user channels. We consider a training-based approach for channel estimation. We provide an information outage probability analysis for the proposed multi-user sharing schemes. 
To My Family 


\section{Acknowledgments}

Many people have supported me during my stay at WVU and a few lines of acknowledgment will not be sufficient to express all my appreciation.

First of all, I owe a special debt of gratitude to my advisor, Dr. Daryl Reynolds for his valuable assistance and timely advice throughout my thesis. I greatly appreciate his patience in answering numerous questions I had while working on this thesis. He has helped me develop and tune my academic skills including research and writing skills. I am really thankful to him and I look forward to continuing my studies under his supervision.

I am grateful to Dr. Matthew Valenti for the many fruitful discussions and his cordial support. I have been deeply touched by his genuine care and concern towards students. I would like to thank him for a myriad help he provided during my studies. I would also like to thank Dr. Arun Ross for being on my committee.

My sincere thanks goes to Dr. Mark Jerabek who gave me the first opportunity to work as a teaching assistant. I would like to give my special thanks to Dr. Ronald Klein for the many helpful discussions and for providing me a fascinating, open and enjoyable working environment while I was his teaching assistant. I would like to thank Dr. Natalia Schmid and Dr. Brian Woerner for giving me a chance to study under their guidance.

I am thankful to my roommates for providing a homelike environment during my stay at WVU. I thank all my friends from WVU as well as from India for their support.

Finally, but most importantly, I thank my mother Geetanjali Vardhe, my father Girish Vardhe, and my brother Shantanu Vardhe for letting me do what I always wanted to do. Their hardwork, sacrifices, unconditional love, trust, patience, and support throughout my career has given me every opportunity to succeed. I would also like to thank the rest of my family including grandparents, uncles, aunts, cousins, and close family-friends for their love and encouragement. I owe my success to my family and as a small token of my gratitude, it is to them I dedicate this work. 


\section{Contents}

Acknowledgments $\quad$ iv

List of Figures $\quad$ vii

List of Tables $\quad$ x

Notation $\quad$ xi

1 Introduction $\quad 1$

1.1 Thesis Outline . . . . . . . . . . . . . . . . . 3

2 Wireless Communication System Overview 5

2.1 Representation of Communication Signals . . . . . . . . . . . . . . 5

2.2 Additive White Gaussian Noise Model . . . . . . . . . . . . . . . . . . 6

2.2.1 Optimum Receiver Design for AWGN channels . . . . . . . . . . . 6

2.2.2 Performance of BPSK in AWGN channel . . . . . . . . . . . . . . . . . 9

2.3 The Wireless Channel . . . . . . . . . . . . . . . . . . . . . . . . . 10

2.3.1 Path Loss and Large Scale Fading . . . . . . . . . . . . . . . . . 11

2.3.2 Small Scale Fading . . . . . . . . . . . . . . . . . . . . . . . . . . . . 12

2.3.3 Time Dispersive Channel . . . . . . . . . . . . . . . . . . . . . 13

2.3.4 Time Varying Channel . . . . . . . . . . . . . . . . . . . . . . . . 14

2.3.5 Rayleigh Fading . . . . . . . . . . . . . . . . . . 15

2.3.6 Performance of BPSK in Flat Fading Rayleigh Channel . . . . . . . . 16

2.4 Summary . . . . . . . . . . . . . . . . . . . . 17

3 Diversity Techniques $\quad 19$

3.1 Introduction . . . . . . . . . . . . . . . . . . . . . . . . 19

3.2 Spatial Diversity and MIMO Communications . . . . . . . . . . . . . 22

3.2.1 Capacity of MIMO Channel . . . . . . . . . . . . . . . 23

3.2.2 The Diversity-Multiplexing Tradeoff . . . . . . . . . . . . . . 25

3.2 .3 The BLAST System . . . . . . . . . . . . . . . . . 26

3.2 .4 Space-Time Coding . . . . . . . . . . . . . . . . 26

3.3 Cooperative Diversity . . . . . . . . . . . . . . . . . . . . . . . . . . 30

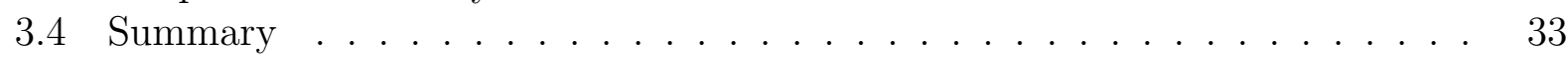


4 Multi-User DF Sharing Schemes with Perfect Inter-User Channels 34

4.1 System Model . . . . . . . . . . . . . . . . . . . 36

4.2 Two-User Decode and Forward Cooperation . . . . . . . . . . . . . . 38

4.3 Three-or-More User Decode and Forward Cooperation . . . . . . . . . . . . . 39

4.4 Receiver Design . . . . . . . . . . . . . . . . . . . . 43

4.5 Amplify and Forward Cooperation . . . . . . . . . . . . . . 46

4.6 Simulation Results . . . . . . . . . . . . . . . . . . . . . . 47

4.7 Conclusion . . . . . . . . . . . . . . . . . . . . . 52

5 Multi-User DF Sharing Schemes with Noisy Inter-User Channels 53

5.1 System Model . . . . . . . . . . . . . . . . . . . . 54

5.2 Two-User Decode and Forward Cooperation . . . . . . . . . . . . . 55

5.3 Three-or-More User Decode and Forward Cooperation . . . . . . . . . . . . . 55

5.4 Receiver Design . . . . . . . . . . . . . . . . . . . . 56

5.5 Simulation Results . . . . . . . . . . . . . . . . . . 60

5.6 Conclusion . . . . . . . . . . . . . . . . . . . . . . 65

6 Information Outage Probability Analysis $\quad 67$

6.1 Outage Events and Outage Probability . . . . . . . . . . . . . . . 68

6.2 System Model . . . . . . . . . . . . . . . . . . . 69

6.3 Laneman's Repetition-Based Cooperative Diversity Protocol . . . . . . . . . 69

6.4 Laneman's Space-Time Coded Cooperative Diversity Protocol . . . . . . . . 70

6.5 Multi-user Cooperation Scheme . . . . . . . . . . . . . . . . . . 71

6.6 Sendonaris's Cooperation Scheme . . . . . . . . . . . . . . . . . 74

6.6 .1 Case I . . . . . . . . . . . . . . . . . . . . . . . . . . . . . . 74

6.6 .2 Case II . . . . . . . . . . . . . . . . . . . . . . . . . . . . . . . . . . 75

6.7 Results . . . . . . . . . . . . . . . . . . . . . 75

6.8 Conclusion . . . . . . . . . . . . . . . . . . . . . . 78

$\begin{array}{lll}7 & \text { Conclusions } & \mathbf{7 9}\end{array}$

7.1 Summary and Conclusions . . . . . . . . . . . . . . . . . . . . 79

7.2 Future Work . . . . . . . . . . . . . . . . . . 80

$\begin{array}{ll}\text { References } & \mathbf{8 1}\end{array}$

A Code Division Multiple Access Technique (CDMA) . . . . . . . . . . . . 85

B Discrete Time Signal Model for Decode and Forward User Cooperation . . . 87

C Derivation for MMSE Filter Design . . . . . . . . . . . . . . . . . 90

D Linear MMSE Channel Estimator . . . . . . . . . . . . . . . . . . . 93

E Information Theory Overview . . . . . . . . . . . . . . . . . 94

F Scalar AWGN Channel Model with Decorrelating Multiuser Detection . . . . 96

G Proof of Information Outage Probability expression for Multi-user Cooperation Scheme . . . . . . . . . . . . . . . . . . . . . . . . . . 98 


\section{List of Figures}

2.1 Decision Region for BPSK . . . . . . . . . . . . . . . . . . . 9

2.2 BER vs SNR performance of BPSK modulation in AWGN channel and flat fading Rayleigh channel. . . . . . . . . . . . . . . 17

3.1 Bit-error-rate for maximum ratio combining with BPSK modulation. . . . . 21

3.2 Ergodic capacity of MIMO system with different antenna configurations. . . 24

3.3 BER performance comparison between BLAST and STBC schemes with BPSK modulation for 2:2 MIMO system. ML detection is used. . . . . . . . . . . 28

3.4 Throuput performance comparison between BLAST and STBC schemes with BPSK modulation for 2:2 MIMO system. The packet size is 256. ML detection is performed. . . . . . . . . . . . . . . . .

3.5 Conventional multi-antenna system array where multiple antennas are implemented on a single unit and are physically connected. . . . . . . . . . . . .

3.6 Distributed multi-antenna system where individual antennas are implemented on a separate mobile unit. There is no physical connection between multiple antennas in this case. . . . . . . . . . . . . . . . . . . . . .

3.7 A simple relay case . . . . . . . . . . . . . . . .

4.1 Bit-error-rate performance of three-user sharing scheme in asynchronous uplink environment when delays are uniformly distributed in the range $\left[0, T_{s}\right]$. Performance of maximum-ratio-combining is also included for comparison.

4.2 Bit-error-rate performance of three-user sharing scheme in asynchronous uplink environment when relative delay ie exactly one symbol interval. Performance of maximum-ratio-combining is also included for comparison. . . . . .

4.3 Block Diagram of the Receiver Structure . . . . . . . . . . . . . . . .

4.4 BER and diversity performance of two-user DF sharing in synchronous and asynchronous uplink environments. The BER curve for the Sendonaris protocol is plotted for comparison, as is the performance of maximum-ratio combining with 2 antennas $(\mathrm{MRC} 2) \ldots \ldots \ldots$

4.5 Throughput performance of two-user DF sharing in synchronous and asynchronous uplink environments. The throughput curve for the Sendonaris protocol and no sharing is plotted for comparison. . . . . . . . . . . . . 
4.6 BER performance of three-user DF sharing in synchronous and asynchronous uplink environments. The BER performance of maximum-ratio combining with three antennas (MRC 3) is provided for comparison.

4.7 Throughput performance of two-user and three-user DF sharing in synchronous and asynchronous uplink environments. Also included is the throughput performance without sharing. . . . . . . . . . . . . . . . . .

4.8 BER performance of two-user and three-user AF sharing in a synchronous uplink. The performance of maximum-ratio combining with 1 and 2 antennas is also included for comparison. . . . . . . . . . . . . . . .

4.9 Throughput performance of two-user and three-user AF sharing in synchronous uplink. Also included is the throughput performance achieved without sharing. 51

5.1 Performance comparison of two training based methods. Three-user sharing protocol operating in synchronous environments with $4 \%$ demodulation error is considered.

5.2 BER performance of three-user DF sharing in a synchronous uplink. The performance of maximum-ratio combining with 2 and 3 antennas is also included for comparison. . . . . . . . . . . . . . . . . .

5.3 Throughput performance of three-user DF sharing in a synchronous uplink. The performance of AF protocol is also included for comparison. . . . . . . .

5.4 BER performance of three-user DF sharing in asynchronous uplink. The performance of maximum-ratio combining with 2 and 3 antennas is also included for comparison.

Throughput performance of three-user DF sharing in asynchronous uplink. .

5.6 BER performance of two-user DF sharing in a synchronous uplink. The performance of maximum-ratio combining with 1 and 2 antennas is also included for comparison.

5.7 Throughput performance of two-user DF sharing in a synchronous uplink. The performance of AF protocol is also included for comparison. . . . . . . .

5.8 BER performance of two-user DF sharing in asynchronous uplink. The performance of maximum-ratio combining with 1 and 2 antennas is also included for comparison. . . . . . . . . . . . . . . . 63

5.9 Throughput performance of two-user DF sharing in asynchronous uplink. . . 64

6.1 Outage probability performance of multi-user cooperation scheme for network size of $\mathrm{m}$ (number of users) $=5$. 'rho' indicates the cross-correlation between spreading codes. Outage probability for Laneman's repetition-based and space-time coded cooperative diversity protocols is also plotted for comparison for same network size. . . . . . . . . . . . . . . .

6.2 Outage probability performance of multi-user cooperation scheme (black color) for network size of $m=2,3, \cdots, 9$ and $\rho=0.7$. Outage probability for Laneman's repetition-based (blue color) and space-time coded (red color) cooperative diversity protocols is also plotted for comparison for same network size. 
6.3 Outage probability performance comparison of multi-user cooperation scheme for network size of $m=2$, and Sendonaris's cooperation scheme. . . . . . . . 77

$.1 \quad$ MMSE Receiver . . . . . . . . . . . . . . . . . . . . . . . . . . . 91 


\section{List of Tables}

4.1 Symbol transmissions from different users in different time slots for three-user DF sharing protocol. . . . . . . . . . . . . . . . . . . 42

4.2 Symbol transmissions from different users in different time slots for two-user AF sharing protocol. . . . . . . . . . . . . . . 46

5.1 Symbol transmissions from different users in different time slots for three-user DF sharing protocol assuming noisy inter-user channel. . . . . . . . . . 56

.1 Symbol transmissions from different users in different time slots for two-user DF sharing protocol. . . . . . . . . . . . . . . . . 87 


\section{Notation}

We use the following notation and symbols throughout this thesis.

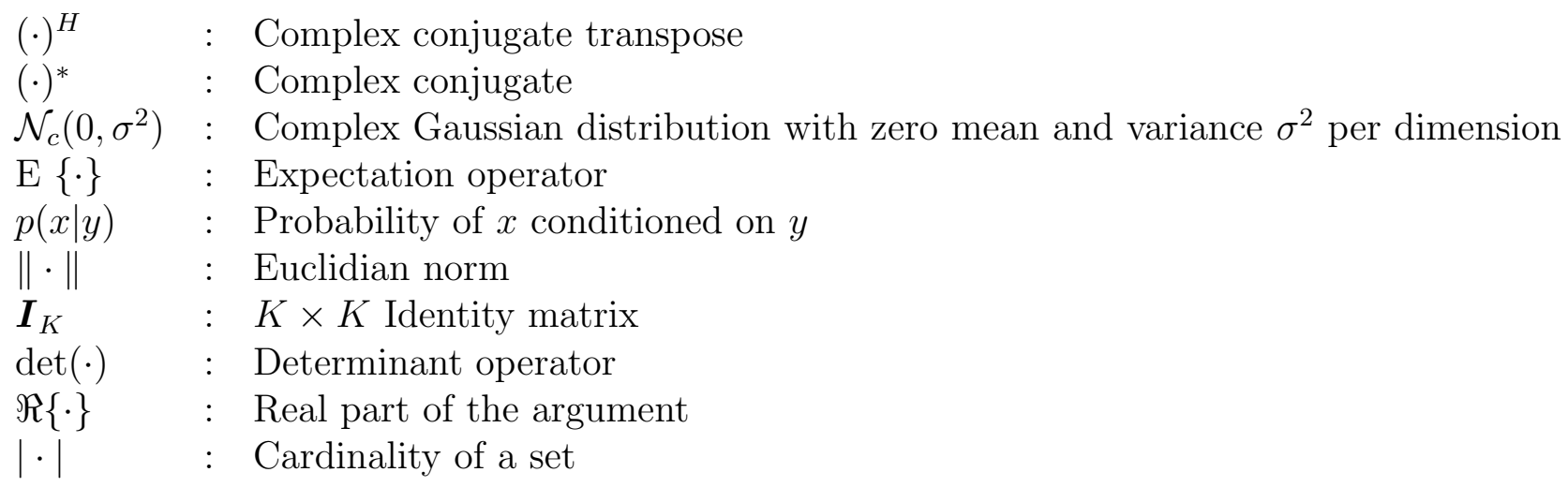

Bold uppercase letters denote matrices while bold lower case letters denote vectors. 


\section{Chapter 1}

\section{Introduction}

As 3G wireless systems get deployed, researchers have begun to explore potential technologies for the $4 \mathrm{G}$ wireless systems. High data rates and high energy efficiency are the demanding requirements of the next generation wireless communication systems. Transmission of video, images requires high data rates and hence data rates as high as $1 \mathrm{~Gb} / \mathrm{s}$ are of interest in the emerging wireless local area networks (WLANs), home audio/video networks. The main cause of reduced data rate or reduced energy efficiency is ' fading'. Due to fading, mobile users in the system go through large variations in the signal attenuation. Signal fades may cause retransmissions in the system causing reduction in the energy efficiency and the data rate. Fading is the phenomenon that distinguishes wired communication and wireless communication. In wired communication, signal-to-noise ratio is easy to predict and doesn't change rapidly making wired communication more reliable. But in mobile wireless communications, fading causes the SNR at the receiver to vary quickly.

There are techniques of SNR control which may improve the system performance. One of the methods is to force the transmitter to transmit at more power when fading is deep and transmit at low power when the fading is not severe. If we allow the transmitter to vary its transmission power such that it maintains constant SNR at the receiver, then the peak-to-average power consumption at the mobile unit tends to infinity [1]. This technique requires channel state information (CSI) at the transmitter which is not practical in a cellular uplink. Battery operated devices will not be able to employ such technique. Another method is to transmit data only when channel conditions are good. This involves delays and also 
estimation of the channel at the transmitter side. Such techniques may not be useful in a cellular uplink. A multiple-input multiple-output (MIMO) system can be used for SNR control through the notion of diversity. A system is said to have a diversity order of $d$ if the average bit-error-rate is proportional to $1 /(\mathrm{SNR})^{d}$. MIMO communications, whereby multiple antennas are implemented at transmitter and/or receiver is the emerging technology to combat fading. We can say that MIMO systems exploit the nature of wireless channel by processing independent fading copies of the same signal. MIMO systems have become popular as they provide dynamic improvements in capacity, data rates and link reliability (because of diversity gains) of wireless systems under the same transmit power budget and bit-error-rate performance requirements as single input single output (SISO) systems.

MIMO communications have applications in the area of cellular networks, wireless ad-hoc type of networks such as wireless sensor networks. To take full advantage of MIMO systems, it is necessary to have uncorrelated fading gains between multiple paths created by multiple transmit and receive antennas in the system. This is possible if the antenna spacing is at least half a wavelength of the transmitted signal. However, for $3 \mathrm{G}$ wireless systems using carrier frequencies of the order of $1 \mathrm{GHz}$, wavelength can not be much smaller than $0.3 \mathrm{~m}$. Due to physical size constraints, implementation of a large number of antennas with many meters dimension is not practical in many situations, such as uplink of the cellular environment, WLANs, or sensor nodes in case of wireless sensor networks. In cellular systems, this would mean deployment of additional base stations in each cluster to increase reliability of data or even deployment of repeaters in addition to access points in case of WLANs, making wireless services costly. Instead, if we allow individual single antenna mobile units to cooperate on information transmission and/or reception, an energy efficient MIMO system can be deployed. As transmit and/or receive antennas which form a MIMO system are not colocated in this type of communication, the technique is in general referred to as 'distributed MIMO communications'.

The broadcast nature (by which transmitted signal is received by multiple nodes at no additional cost) of the wireless medium and its ability to achieve diversity through independent paths are the two key features which contribute to the cooperative communication. The basic idea as applied to ad-hoc networks can be briefly discussed as follows. Ad-hoc networks 
do not operate in a infrastructure mode and hence do not employ a central monitoring unit. On the availability of data, mobile units send information to a desired destination. Other mobile units in the network hear all the transmissions because of the broadcast nature of the wireless channel. These mobile units then cooperate and resend this information which is combined and processed at the destination node. The appropriate processing at the destination node exploits the inherent diversity of the channel. In this case, each mobile unit becomes part of the distributed antenna array. The same idea can be applied to a cellular uplink in which we assume the base station as a desired destination.

\subsection{Thesis Outline}

In this thesis, we focus on distributed MIMO communications which has applications in wireless ad-hoc networks, cellular architectures. Particularly, we discuss cooperative diversity sharing protocols for the cellular uplink though the same concept can be applied to wireless ad-hoc networks.

Most of the recent work on cooperative diversity considers variations of half duplex and full duplex sharing protocols from an information-theoretic point of view. In [2], the authors consider decode and forward (DF) and amplify-forward (AF) protocols which are half duplex and they develop an analytical performance characterization in terms of outage probabilities. In [3], [4], the authors provide high-level descriptions of multiuser sharing strategies that achieves the diversity-multiplexing tradeoff. However, none of these works presents specific coding schemes, modulations, or receiver designs, and they do not investigate performance in practical environments. In [5], [6], the authors develop a specific two-user sharing protocol for the CDMA uplink, but the assumption of orthogonal codes and other design constraints limit the performance and flexibility of the scheme. In this thesis, we propose data sharing schemes that simultaneously provide full diversity, as measured by the bit-error-rate (BER) slope, and high throughput, as compared to the Sendonaris scheme $[5,6]$ and to a conventional nosharing uplink. Our receivers are restricted to linear designs that jointly suppress multiuser interference and perform space-time decoding. They have the advantages of low complexity and they are easy to make adaptive. We have chosen to apply the schemes to a code division 
multiple access (CDMA) uplink because it allows us to investigate the affects of multiuser interference in a simple way. CDMA has also been accepted in the US and Europe for the next generation wireless communication systems (cdma2000 and WCDMA respectively) and hence it is of interest to us. However, the sharing schemes can be applied to time or frequency division multiplexing systems with little modification. We consider both synchronous as well as asynchronous communication scenarios. Cooperative diversity with noisy inter-user channels has been studied in $[7,8]$. But the prior work is restricted to two-user sharing and synchronous communication environments and does not specify the receiver structure. Here, we present an adaptive receiver structure and additional bit-error-probability and throughput simulations for practical, noisy inter-user channels. We provide an informationoutage probability analysis of the multi-user sharing schemes.

The thesis continues as follows. Chapter 2 gives an overview of the wireless communication systems. It explains the additive white Gaussian channel model which is a very basic channel model to any communication system. It also addresses issues related to wireless channel such as large scale and small scale fading. Chapter 3 introduces various diversity techniques available to improve reliability of the wireless link. In particular, it talks about key areas related to this thesis such as spatial diversity through MIMO channels, decode and forward (DF) protocol and amplify and forward (AF) protocols for the cooperative diversity. In Chapter 4, we propose cooperative diversity data sharing protocols (two-user and three-and-more user DF data sharing schemes) for the cellular uplink. We provide a system model, practical linear receiver design and also simulation results assuming the case of perfect inter-user channels (i.e. we assume mobile users successfully decode other user's data hence there are no decoding errors at the user side). We also present simulation results for the two-user and three-user AF protocol. Chapter 5 discusses the more practical situation where inter-user channel is noisy and hence mobile units introduce decoding errors before retransmitting other user's data. Here, we present an adaptive receiver design based on the channel estimation technique. Chapter 6 contains analytical outage probability results. Finally, Chapter 7 concludes with some insights to future research. 


\section{Chapter 2}

\section{Wireless Communication System Overview}

\subsection{Representation of Communication Signals}

The purpose of a communication system is to convey an information bearing signal from source to destination through physical channel. Modulation is the process required to transform a signal into a suitable form that can be transmitted over a desired channel. Let' s consider a information bearing low pass signal $s(t)$ with its frequency contents centered around $0 \mathrm{~Hz}$. In general, $s(t)$ is a complex-valued quantity. The equivalent band pass signal (after modulation) $x(t)$ having its frequency contents centered around carrier frequency $f_{c}$ $\mathrm{Hz}$ can then be represented as

$$
x(t)=\operatorname{Re}\left[s(t) e^{j 2 \pi f_{c} t}\right]
$$

At the receiver a reverse process called demodulation is carried to out to recover the information bearing signal $s(t)$. The mathematical relationship between low pass and band pass signal allows us to ignore any linear frequency translations experienced during the modulation process [9]. Also, we note that the performance of various modulation and demodulation techniques does not alter with the carrier frequency. Hence, throughout this thesis, we deal with only equivalent low pass signals. 


\subsection{Additive White Gaussian Noise Model}

The transmitted signal encounters noise introduced by the communication channel. The noise which is random makes the communication process probabilistic in nature. Thermal noise, produced by the random motion of electrons in conducting media and shot noise, produced by random fluctuations of current in electronic devices are the common sources of noise. Since this noise is present over all the frequencies of interest, the term white noise is used to describe it. White noise has the power spectral density independent of the operating frequency which is denoted by a constant $\frac{N_{0}}{2}$. Autocorrelation function of white noise which is nothing but the inverse fourier transform of the power spectral density is then given by $R(\tau)=\frac{N_{0}}{2} \delta(\tau)$. Hence it is clear that the samples of white noise process taken at different times are uncorrelated. The white noise is often well modelled by a Gaussian distribution. This can be justified by central limit theorem which says that distribution of sum of independently and identically distributed random variables approaches that of Gaussian distribution if this number is large enough ${ }^{1}[10]$.

The complex-valued received signal under additive white Gaussian noise (AWGN) channel is given by

$$
r(t)=s(t)+n(t)
$$

where samples of the continuous time white Gaussian noise process $n(t)$ are independent (since for Gaussian process, uncorrelated random variables implies independent random variables) with mean 0 and variance $\frac{N_{0}}{2}$.

\subsubsection{Optimum Receiver Design for AWGN channels}

Suppose one of the $M$ possible signals $s_{i}(t)$ from the set $\left\{s_{1}(t), s_{2}(t), \ldots \ldots, s_{M}(t)\right\}$ is transmitted which gets corrupted due to white Gaussian noise. Specifically, we consider the transmission of information over the interval $0 \leq t \leq T$. The receiver's job is then to estimate the transmitted signal based upon the observation $r(t)$. This section explains the design of optimum receiver [11]. The receiver is optimum in the sense that it minimizes the

\footnotetext{
${ }^{1}$ In practice, the number 10 is usually enough to see this effect
} 
probability of error which is the most common optimality criterion for digital communication systems. The receiver can be divided into two blocks: the demodulator and the detector. The demodulator converts the continuous time received signal into discrete time signal vector (or we say that it generates sufficient statistics) and the detector makes a good estimate of the transmitted signal. The demodulator can be implemented in the form of a correlation demodulator or a matched filter demodulator. Here, we consider a correlation demodulator but we also note the important property of matched filter that it maximizes the output signal-to-noise ratio (SNR).

We follow the signal space approach and present the structure of optimal receiver. The transmitted signal can be represented by a linear combination of orthonormal basis functions:

$$
s_{i}(t)=\sum_{k=1}^{K} s_{i, k} f_{k}(t), \quad i=1, \ldots M
$$

The functions $\left\{f_{1}(t), f_{2}(t), \ldots, f_{K}(t)\right\}$ form a complete orthonormal basis for the signal set ${ }^{2}$ over the interval $0 \leq t \leq T$.

\section{The Correlation Demodulator}

The received signal $r(t)$ is passed through a parallel bank of $K$ correlators which compute the projection of $r(t)$ onto the $K$ orthonormal basis functions. Thus, the demodulator output can be written as

$$
r_{k}=s_{i k}+n_{k}, \quad k=1,2, \ldots, K
$$

where

$$
\begin{aligned}
& r_{k}=\int_{0}^{T} r(t) f_{k}(t) \\
& s_{i k}=\int_{0}^{T} s_{i}(t) f_{k}(t) \\
& n_{k}=\int_{0}^{T} n(t) f_{k}(t)
\end{aligned}
$$

\footnotetext{
${ }^{2}$ complete orthonormal basis set can be found using Gram-Schmidt Orthogonalization procedure.
} 
We note here that the basis functions $\left\{f_{k}(t)\right\}$ do not span the noise space. Then

$$
n^{\prime}(t)=n(t)-\sum_{i=1}^{K} n_{k} f_{k}(t)
$$

represents the difference between the original noise process and the projection of $n(t)$ onto the orthonormal basis functions $f_{k}(t)$. The continuous time received signal in this case can be expressed as

$$
r(t)=\sum_{i=1}^{K} r_{k} f_{k}(t)+n^{\prime}(t) .
$$

It can be be shown that the noise $n^{\prime}(t)$ can be disregarded by the receiver since it is irrelevant to the decision on the transmitted signal [9]. Thus $r_{k}$ 's are said to be sufficient statistics as they contain sufficient information to make a decision at the detector on which of the $M$ signals was transmitted.

\section{The Optimum Detector}

Using the signal space approach as discussed in the above section, we were able to reduce the decision to a finite dimensional space. We can now say that we transmit a vector $\boldsymbol{s}_{i}=\left[s_{i 1}, s_{i 2}, \ldots s_{i K}\right]$ and we receive a vector $\boldsymbol{r}=\left[r_{1}, r_{2}, \ldots, r_{K}\right]=\boldsymbol{s}_{i}+\boldsymbol{n}$, where elements of the vector $\boldsymbol{n}$ are i.i.d (independent and identically distributed) Gaussian random variables. Given $\boldsymbol{r}$, detector's job is to form an estimate $\hat{\boldsymbol{s}}_{i}$ of the transmitted signal vector which minimizes the probability of symbol error. The detector implementing the maximum a posteriori probability (MAP) decision rule is the optimum detector since it minimizes the symbol error probability by choosing the signal $\boldsymbol{s}_{i}$ which satisfies

$$
\operatorname{Pr}\left(\boldsymbol{s}_{i} \mid \boldsymbol{r}\right) \geq \operatorname{Pr}\left(\boldsymbol{s}_{m} \mid \boldsymbol{r}\right), \quad \forall i \neq m
$$

Or equivalently,

$$
\frac{p\left(\boldsymbol{r} \mid \boldsymbol{s}_{i}\right) \operatorname{Pr}\left(\boldsymbol{s}_{i}\right)}{p(\boldsymbol{r})} \geq \frac{p\left(\boldsymbol{r} \mid \boldsymbol{s}_{m}\right) \operatorname{Pr}\left(\boldsymbol{s}_{m}\right)}{p(\boldsymbol{r})}, \quad \forall i \neq m
$$

If $\operatorname{Pr}\left(s_{1}\right)=\ldots=\operatorname{Pr}\left(s_{M}\right)$ or the a priori probabilities are unknown, then the MAP rule simplifies to maximum likelihood (ML) decision rule. For the AWGN channel, ML decision rule finds the signal $\boldsymbol{s}_{i}$ that is closest in distance ${ }^{3}$ to the received signal vector $\boldsymbol{r}$.

\footnotetext{
${ }^{3}$ Here, by distance, we mean Euclidian distance. The Euclidian distance $\boldsymbol{D}\left(\boldsymbol{r}, \boldsymbol{s}_{i}\right)=\sum_{i=1}^{K}\left(r_{k}-s_{i k}\right)^{2}$.
} 


\subsubsection{Performance of BPSK in AWGN channel}

In case of a binary phase shift keying (BPSK) modulation, one of the following two equiprobable signals is transmitted.

$$
s_{1}(t)=\sqrt{E_{b}} f_{1}(t), \quad s_{2}(t)=-\sqrt{E_{b}} f_{1}(t), \quad 0 \leq t \leq T .
$$

where $E_{b}$ is the signal energy per bit. The signal space representation is given by

$$
s_{1}=\sqrt{E_{b}}, \quad s_{2}=-\sqrt{E_{b}} .
$$

The received symbol in this case is

$$
r= \pm \sqrt{E_{b}}+n
$$

where $n \sim \mathcal{N}\left(0, \frac{N_{0}}{2}\right)$. Since the symbols are equiprobable, ML decision rule is the optimum and says, choose $s_{1}$ if

$$
\begin{aligned}
& p\left(r \mid s_{1}\right) \geq p\left(r \mid s_{2}\right) \\
& \Leftrightarrow \frac{1}{\sqrt{\pi N_{0}}} \exp \left\{-\frac{\left(r-\sqrt{E_{b}}\right)^{2}}{N_{0}}\right\} \geq \frac{1}{\sqrt{\pi N_{0}}} \exp \left\{-\frac{\left(r+\sqrt{E_{b}}\right)^{2}}{N_{0}}\right\} \\
& \Leftrightarrow r \geq 0 \\
& \begin{array}{c|c}
R_{2} & R_{1} \\
\hline S_{2}=-\sqrt{E_{b}} & S_{1}=\sqrt{E_{b}}
\end{array}
\end{aligned}
$$

Figure 2.1: Decision Region for BPSK

\section{Probability of Error for BPSK}

From Fig. 2.1, given that $s_{2}$ was transmitted, the probability of error is simply the probability that $r \geq 0$ where $r \sim \mathcal{N}\left(E_{b}, \frac{N_{0}}{2}\right)$. 


$$
\begin{aligned}
\operatorname{Pr}\left[\hat{s} \neq s_{2} \mid s=s_{2}\right] & =1-\int_{-\infty}^{0} \frac{1}{\sqrt{\pi N_{0}}} \exp \left(-\frac{\left(r+\sqrt{E_{b}}\right)^{2}}{N_{0}}\right) d r \\
& =Q\left(\sqrt{\frac{2 E_{b}}{N_{0}}}\right)
\end{aligned}
$$

By symmetry,

$$
\operatorname{Pr}\left[\hat{s} \neq s_{1} \mid s=s_{1}\right]=\operatorname{Pr}\left[\hat{s} \neq s_{2} \mid s=s_{2}\right]
$$

Hence,

$$
\text { Probability of symbol error }=Q\left(\sqrt{\frac{2 E_{b}}{N_{0}}}\right)
$$

where, $\frac{E_{b}}{N_{0}}$ is nothing but the signal-to-noise ratio (SNR) at the receiver ${ }^{4}$. For BPSK, symbol error probability and bit error probability are the same.

\subsection{The Wireless Channel}

The AWGN channel model described in the last section is an ideal channel model for wireless communication systems and is sometimes used to understand its basic performance. The more practical channel model considers the effects of fading which represents fluctuations in the instantaneous received signal strength due to multipath propagation of the transmitted signal. As the signal travels from the transmitter to receiver, it gets reflected by various objects and hence travels along multiple paths. At the receiver, these multipath components add constructively or destructively depending upon the attenuation factor and their phase angles causing received signal strength to fluctuate with time and distance. Multipath fading can be considered as the significant difference between wired communications and wireless communications. The three basic propagation mechanisms that affect the signal propagation characteristics in the mobile communication systems are reflection, diffraction and scattering. Reflection occurs when the propagating signal wave strikes the surface of

\footnotetext{
${ }^{4} \mathrm{SNR}=\frac{\text { signal power }}{\text { noise power }}=\frac{E b / T_{b}}{N_{0} B}$ where, $E_{b}$ is the energy per bit, $T_{b}$ is one bit duration and $B$ is the signal bandwidth
} 
an object having dimension larger than the signal wavelength. Diffraction occurs when the signal path is obstructed by an object having sharp edges. Scattering occurs when the propagation medium consists of objects with dimensions smaller than the signal wavelength. All the three propagation mechanisms depend on the amplitude, phase and polarization of the incident electromagnetic wave and also the geometry of the object [12]. Large scale fading and small scale fading are the two main types of fading which can be well described by the above mentioned propagation mechanisms.

\subsubsection{Path Loss and Large Scale Fading}

As the distance between transmitter and receiver increases, there is a gradual decrease in the average received power. This gradual large-scale variation in the signal strength which is simply a function of transmitter-receiver separation is termed as 'large scale fading'. Large scale fading statistics helps us compute an estimate of the path loss as a function of distance. Large scale propagation models are often used in the link budget analysis in a cellular application. Link budget analysis can then be used to predict SNR at the mobile units and to estimate the coverage area for a particular mobile communication systems.

\section{Log-Distance Path Loss Model}

Based on theoretical and empirical propagation models, it is seen that the mean received signal strength decreases logarithmically with distance for indoor and outdoor wireless channels. Free space path loss is proportional to the square of the distance while with perfect ground reflection, the average received power falls off according to the fourth power of distance [13]. But in actual practice, the average large-scale path loss is given by

$$
\overline{P L}(d)=\overline{P L}\left(d_{0}\right)+10 n \log \left(\frac{d}{d_{0}}\right) \mathrm{dB}
$$

where $d$ is the T-R separation distance, $d_{0}$ is the reference distance in the far field of the antenna, $n$ is the path loss exponent whose value depends on the antenna height, frequency and the propagation environment [14]. Measurements show that 2 (free space) < $n<10$ (highly densed environment). The path loss at a reference distance $d_{0}$ is usually found using field measurements. 


\section{Log-normal Shadowing}

The above path loss model gives the average path loss and ignores the fact that two different locations having the same T-R separation could receive the same signal at quite different powers. But from the measurements, it can be concluded that the path loss at a particular distance is random and is said to be log-normally distributed about its mean value $\overline{P L}(d)$. Thus,

$$
\overline{P L}(d)=\overline{P L}\left(d_{0}\right)+10 n \log \left(\frac{d}{d_{0}}\right)+X_{\sigma} \mathrm{dB}
$$

where $X_{\sigma} \sim \mathcal{N}\left(0, \sigma^{2}\right)$. In this thesis, we assume that the problem of large scale fading has been alleviated by the addition of 'fade margin' in the link budget analysis. Fade margin is nothing but the additional signal power needed to overcome shadowing effect with specific probability.

\subsubsection{Small Scale Fading}

As mobile units move over a very short distance, the received signal strength may fluctuate rapidly. This rapid fluctuation in the received signal power over a short period of time or distance, is termed 'small-scale fading'. Due to basic propagation mechanisms, multipath components of a signal arrive at the receiving antenna with different propagation delays and amplitudes. A mobile radio multipath channel can be characterized by a linear time-variant filter. The time-variant impulse response of the mobile channel can be expressed as

$$
h(\tau ; t)=\sum_{n} \alpha_{n}(t) e^{-j 2 \pi f_{c} \tau_{n}(t)} \delta\left(\tau-\tau_{n}(t)\right)
$$

where $f_{c}$ is the carrier frequency, $\alpha_{n}(t)$ and $\tau_{n}(t)$ are the complex attenuation factor and propagation delay of the $n$-th path. The transfer function of the time-variant channel can be written as

$$
H(f ; t)=\int_{\infty}^{-\infty} h(\tau ; t) e^{-j 2 \pi f \tau} d \tau
$$

The time-varying filtering nature of the channel accounts for small scale fading. It can be described in terms of the two mechanisms: 
1. Time dispersion caused by frequency variations in $H(f ; t)$;

2. Frequency dispersion caused by time variations in $H(f ; t)$.

\subsubsection{Time Dispersive Channel}

The autocorrelation of the transfer function is

$$
\phi_{H}(\triangle f ; \triangle t)=E\left[H^{*}(f ; t) H(f+\triangle f ; t+\triangle t)\right] .
$$

$\phi_{H}(\triangle f ; \triangle t)$ is also called as spaced-frequency, spaced-time correlation function of the channel.

Frequency correlation function of the channel is obtained by substituting $\Delta t=0$ in (2.26). Let

$$
\phi_{H}(\triangle f) \triangleq \phi_{H}(\triangle f ; 0)
$$

The multipath intensity profile (or power-delay profile) can then be obtained as

$$
\phi_{h}(\tau)=F^{-1}\left\{\phi_{H}(\triangle f)\right\}
$$

The multipath intensity profile refers to the average received power as a function of time delay $\tau$. The range over which $\phi_{h}(\tau)$ is non-zero is called the maximum delay spread $\left(T_{m}\right)$ of the channel. And the width over which $\phi_{H}(\triangle f)$ is non-zero is called the coherence bandwidth $\left(B_{m}\right)$ of the channel. The maximum delay spread and the coherence bandwidth of the channel are related by

$$
B_{m} \approx \frac{1}{T_{m}}
$$

If the bandwidth of the transmitted signal $\left(B_{s}\right)$ is less than the coherence bandwidth of the channel, the channel gain is constant over the entire signal bandwidth and the signal is said to undergo flat fading. If coherence bandwidth of the channel is less than the bandwidth of the transmitted signal, different frequency components of the signal undergo different fading attenuation and delays and this type of fading is called frequency selective fading. Similar interpretation for frequency selectivity of the channel can be given as follows. When 
$T_{m}>T_{s}$ (symbol period), i.e when multipath components of the transmitted signal arrive beyond the symbol's duration, the channel is said to exhibit frequency selective fading. Such time dispersion results in the channel-induced inter-symbol interference (ISI). When $T_{m}<T_{s}$, all the signal components arrive within symbol's duration, there is no channel induced ISI and the channel is said to be flat fading. Equivalently, the coherence bandwidth is the range of frequencies over which the channel can be considered flat. The multipath spread, on the other hand, indicates the level of time dispersion (time spreading) caused by the frequency selective nature of the channel.

\subsubsection{Time Varying Channel}

The impulse response of the channel changes with time due to relative motion between transmitter and receiver. The parameters like Doppler spread and coherence time are used to describe the time varying nature of the channel. Taking into account the spaced-frequency, spaced-time correlation function, we now concentrate on the time variations in the channel as measured by the parameter $\triangle t$ in $\phi_{H}(\triangle f ; \triangle t)$. Let

$$
S_{H}(\triangle f ; \lambda)=\int_{-\infty}^{\infty} \phi_{H}(\triangle f ; \triangle t) e^{-j 2 \pi \lambda \triangle t} d \triangle t
$$

denote the Fourier Transform of $\phi_{H}(\triangle f ; \triangle t)$ with respect to the $\triangle t$ domain. The Doppler power spectrum of the channel is obtained by substituting $\triangle f=0$ in (2.30). Let

$$
S_{H}(\lambda) \triangleq S_{H}(0 ; \lambda)
$$

denote the Doppler power spectrum of the channel which gives the signal intensity as a function of the doppler frequency $(\lambda)$. The doppler power spectrum is also the Fourier Transform of the time correlation function $\phi_{H}(\triangle t)$ of the channel. The range over which $S_{H}(\lambda)$ is non-zero is called the Doppler spread $\left(B_{d}\right)$ of the channel. The coherence time $\left(T_{c}\right)$ which indicates the time duration over which different received signals have a strong potential of amplitude correlation and the Doppler spread are related by

$$
T_{c} \approx \frac{1}{B_{d}} .
$$


If the coherence time of the channel is greater than the symbol period, the channel is called slow fading otherwise it is called fast fading. In the fast fading case, the channel impulse response changes rapidly within the symbol duration. A frequency domain interpretation of the time variance of the channel can be given as follows. When $B_{s}<B_{d}$, the channel is called fast fading channel otherwise it is recognized as a slow fading channel. If the baseband signal bandwidth is much greater than $B_{d}$, the effects of Doppler spreading are negligible at the receiver.

\subsubsection{Rayleigh Fading}

The discrete time signal model for slowly varying, flat fading channel can be written as

$$
\boldsymbol{r}=h \boldsymbol{b}+\boldsymbol{n}
$$

where $\boldsymbol{b}$ is the transmitted data vector, $\boldsymbol{n} \sim \mathcal{N}_{c}\left(0, \sigma^{2} \boldsymbol{I}\right)$ and $h$ is the complex channel gain.

$$
h=h_{i}+h_{q}=\alpha \exp (j \phi) .
$$

The channel gains $h$ are random and since each channel gain is due to the effect of superposition of large number of random effects, by central limit theorem, they can be modelled as zero mean complex Gaussian random variables (assuming no line of sight component). In this case, the fading amplitude $\alpha=\sqrt{h_{i}^{2}+h_{q}^{2}}$ will be Rayleigh distributed. Also, the phase of the fading coefficient is uniformly distributed over $[0,2 \pi]$. When there is strong line of sight component present, the envelope of the received signal follows Rician distribution.

If the channel fading coefficient remains constant over a block of data and then changes independently between blocks, then such type of fading is called as 'block fading'. For example, suppose a block consists of $N$ symbols each with time duration $T$. Then the baseband received signal in a block fading can be written as

$$
r(t)=h s(t)+n(t) \quad 0<t \leq N T
$$

where $s(t)$ is the baseband transmitted signal, $h$ is the complex Gaussian random variable which does not change during the block but changes independently during the second block, $N T<t \leq 2 N T$.

Throughout this thesis, we will use the block fading Rayleigh channel model. 


\subsubsection{Performance of BPSK in Flat Fading Rayleigh Channel}

Consider a discrete time signal model same as in equation (2.33) for BPSK modulation over flat fading channel. In this case $\boldsymbol{b} \in\{+1,-1\}$ represents the BPSK symbol vector, $h$ is a scalar, zero mean complex Gaussian fading coefficient that remains constant over a block of symbols and varies independently between blocks. To compensate for the phase changes in the signal due to channel and to collect all possible signal energy, we multiply the received signal by the complex conjugate of the fading coefficient (here we assume coherent detection and hence the channel is known at the receiver). The decision statistics in this case is

$$
\boldsymbol{d}=h^{*} \boldsymbol{r}
$$

and the bit decisions are

$$
\hat{\boldsymbol{b}}=\operatorname{sign}\{\Re[\boldsymbol{d}]\} .
$$

The probability of error in slow flat-fading channels can be obtained by taking expectation of (2.21) with respect to the probability density of the instantaneous fading SNR. The probability of error for BPSK modulation in a slow fading channel can then be evaluated as

$$
P_{e}=\int_{0}^{\infty} P_{e}(\gamma) p(\gamma) d \gamma
$$

where $P_{e}(\gamma)=Q(\sqrt{2 \gamma})$ is the probability of error for BPSK modulation at a specific value of signal-to-noise ratio $\gamma$ and $p(\gamma)$ is the probability density function of SNR due to the fading channel. For Rayleigh fading channels, the fading power $|h|^{2}$ and hence the SNR $\gamma$ have an exponential distribution. Therefore,

$$
p(\gamma)=\frac{1}{\Gamma} \exp \left(-\frac{\gamma}{\Gamma}\right)
$$

where $\Gamma=\frac{E_{b}}{N_{0}}|\bar{h}|^{2}$ is the average SNR. The average BER in the Rayleigh fading channel is

$$
\text { probability of error }=\int_{0}^{\infty} Q(\sqrt{2 \gamma}) \frac{1}{\Gamma} \exp \left(-\frac{\gamma}{\Gamma}\right) d \gamma
$$

It can be shown that for coherent BPSK, the probability of error in a slow fading channel evaluates to [12],

$$
\text { probability of error }=\frac{1}{2}\left[1-\sqrt{\frac{\Gamma}{1+\Gamma}}\right]
$$


At high SNRs,

$$
\text { probability of error }=\frac{1}{4 \Gamma} \text {. }
$$

We observe that there exists an inverse algebraic relationship between bit-error-rate and SNR in case of fading channels while AWGN channels exhibit an exponential relationship between bit-error-rate and SNR. Hence, to achieve the same value of BER for any modulation technique in fading channels as AWGN channels, a significant increase in the received SNR is required. That means, transmission of signals over the fading channels would require much more transmit power as compared to AWGN channels. There exist techniques to reduce the effect of fading. These include error control coding and diversity techniques which we will discuss in the next chapter.

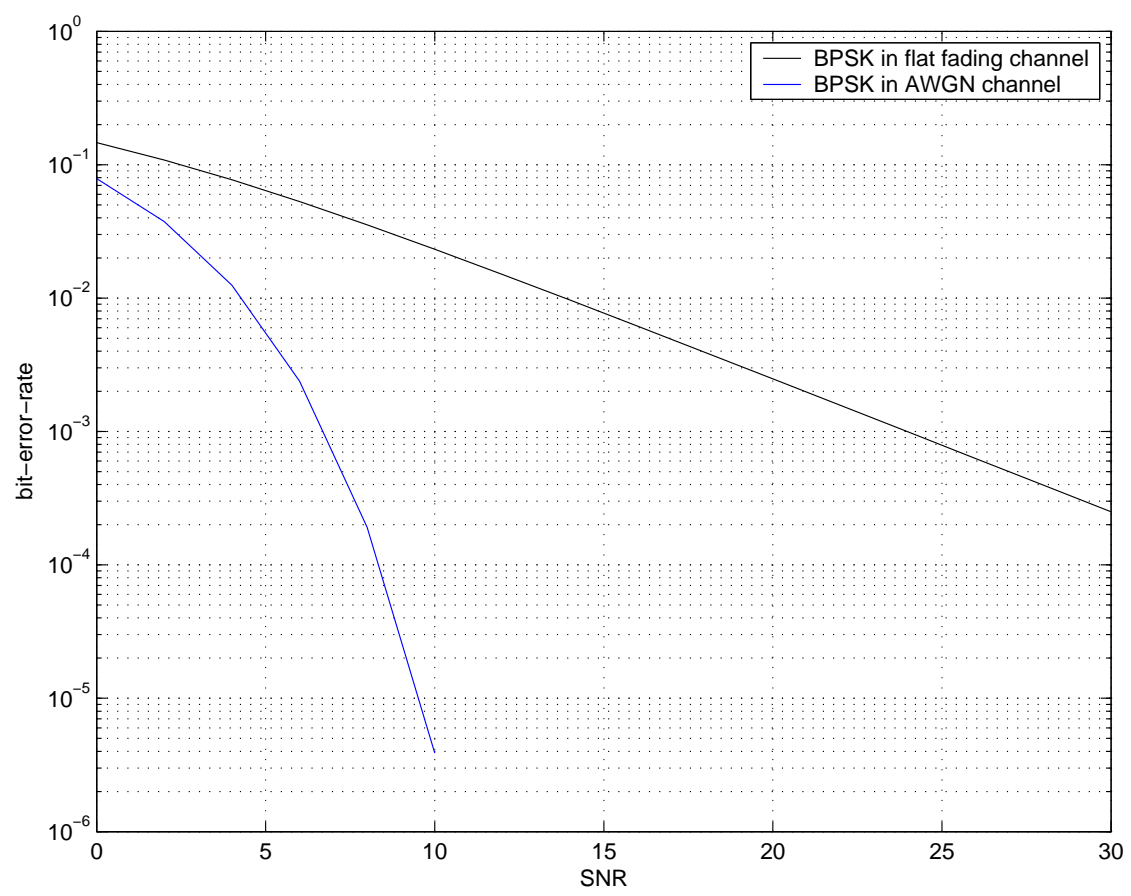

Figure 2.2: BER vs SNR performance of BPSK modulation in AWGN channel and flat fading Rayleigh channel.

\subsection{Summary}

The MAP detector is the optimum detector for AWGN channels since it minimizes the probability of symbol error. The practical channel model for the wireless communication 
system takes into account the effects of fading. Large scale fading and small scale fading are the main types of fading. The wireless channel can be classified as (i) flat fading channel, (ii) frequency selective channel, (iii) slow fading channel and (iv) fast fading channel. The performance of a wireless system drastically degrades in the presence of fading unless proper signal processing techniques are employed. 


\section{Chapter 3}

\section{Diversity Techniques}

\subsection{Introduction}

As we saw in the last chapter, fading is the main channel impairment that reduces both the data rate and energy efficiency of the wireless systems. Diversity is a well known technique which exploits the multipath nature of the wireless channel by finding independent (highly uncorrelated) signal paths and is a very effective technique to combat fading. The concept of diversity can be explained as follows. Suppose $p$ is the probability that a single path suffers from high signal attenuation due to fading coefficient over that branch and let there are total $L$ independent paths (or branches) between transmitter and receiver. Then the probability that all paths experience a deep fade is $p^{L}$ under the assumption of uncorrelated fading. Thus, by exploiting the multipath nature of the channel, the average SNR at the receiver may be improved, and thus improving the reliability of the signal. Various methods to achieve diversity exist including time diversity, frequency diversity, polarization diversity and spatial diversity. Time diversity adds redundant data bits in the time domain. The time intervals during which redundant information is sent exceed the coherence time of the channel providing multiple independent faded copies of the signal to the receiver. Channel coding may be viewed as a form of time diversity. The RAKE receiver is a recent implementation of time diversity for CDMA. But unlike channel coding, RAKE receiver only provides time diversity when there exist multiple resolvable paths. Frequency diversity sends information symbols over multiple carrier frequencies. Here, the carrier frequencies are separated by at least the 
coherence bandwidth of the channel and hence experience independent fades. An example of frequency diversity is multicarrier modulation. Polarization diversity involves implementing orthogonally polarized co-located antennas at the mobile units. Spatial diversity (also called antenna diversity) can be achieved by implementing multiple antennas at the transmitter side or the receiver side or both at the transmitter and receiver side (multiple-input multipleoutput communications). We will look at spatial diversity in more detail in the next sections.

\section{SNR Improvement Due to Selection Diversity}

Let us assume that the receiver observes $M$ faded copies of the same signal through independent channels. These channels might be available because of time diversity, frequency diversity or spatial diversity. Let $\Gamma$ be the average SNR of each channel. Then it can be shown that the average SNR at the receiver due to selection diversity where receiver selects the channel with the highest SNR is [15], [12]

$$
\bar{\Gamma}=\Gamma\left(1+\sum_{k=2}^{M} \frac{1}{k}\right) .
$$

Thus, the SNR improvement due to selection diversity is

$$
\frac{\bar{\Gamma}}{\Gamma}=\sum_{k=1}^{M} \frac{1}{k} .
$$

Selection diversity technique ignores the information from all other channels except one, and hence this is not the best technique but is easy to implement.

\section{SNR Improvement Due to Maximum Ratio Combining Technique}

Maximum ratio combining (MRC) technique coherently adds the information over all the channels and provides a maximum SNR at the receiver. Again, suppose, $\Gamma$ is the average SNR of each channel, then the average SNR at the receiver employing MRC technique is [12]

$$
\bar{\gamma}=M \Gamma .
$$

As can be seen from the above formula, in this method, signals from all $M$ branches are weighted according to respective channel coefficients and then added. Thus, even if none of 
the branches have an average SNR above some threshold, MRC could produce an output having SNR value above the specified threshold. The probability of error $(\operatorname{Pr}(e))$ for BPSK modulated system in AWGN channel and having $M$ branch diversity with MRC is given by $[15]$

$$
\operatorname{Pr}(e)=\left[\frac{1}{2}(1-\mu)\right]^{M} \sum_{k=0}^{M-1}\left(\begin{array}{c}
M-1+k \\
k
\end{array}\right)\left[\frac{1}{2}(1+\mu)\right]^{M}
$$

where

$$
\mu=\sqrt{\frac{\Gamma}{1+\Gamma}}
$$

At $\Gamma>10 \mathrm{~dB}$, using high SNR approximation,

$$
\operatorname{Pr}(e) \cong\left(\frac{1}{4 \Gamma}\right)^{M}\left(\begin{array}{c}
2 M-1 \\
M
\end{array}\right)
$$

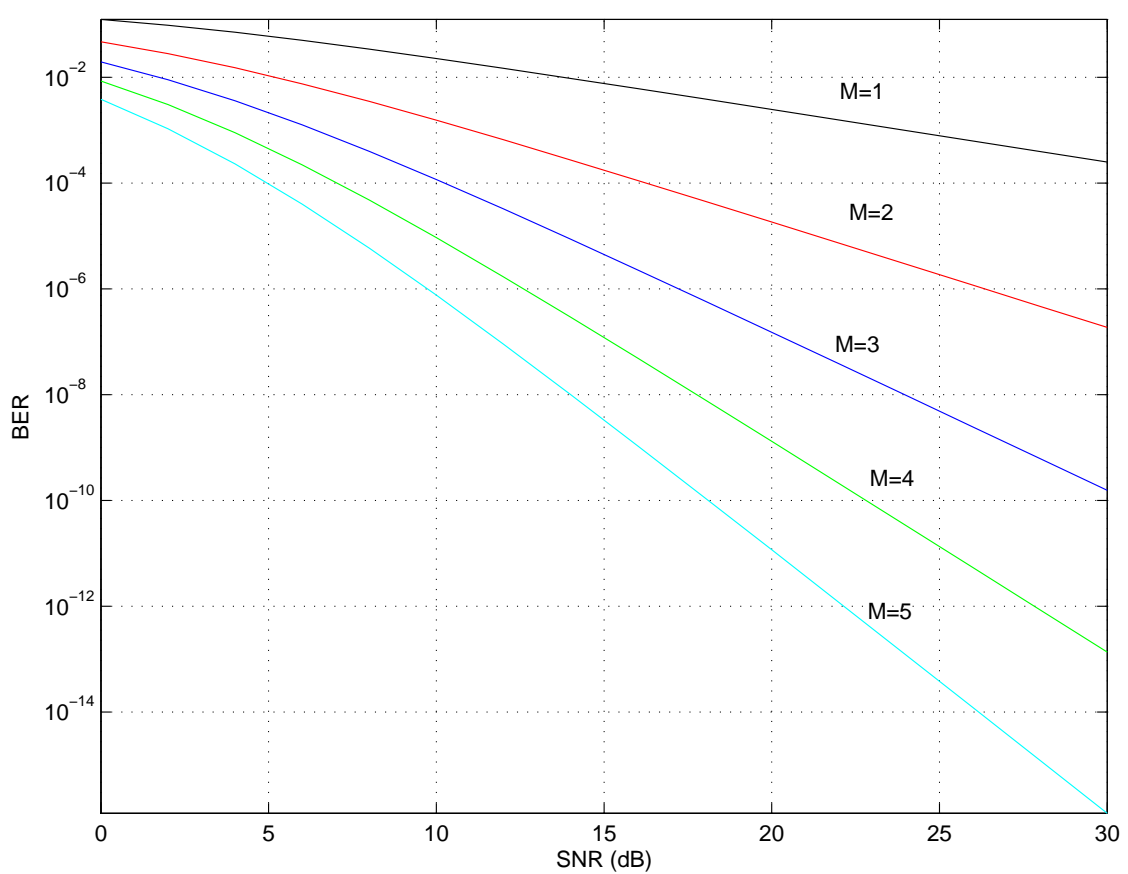

Figure 3.1: Bit-error-rate for maximum ratio combining with BPSK modulation.

Fig. 3.1 indicates the relationship between SNR and BER for varied number of diversity branches. It can be seen that increasing the number of diversity branches increases the slope of the BER curve, which is nothing but a diversity gain. From Chapter 2, we know 
that there exists a linear relationship (in the log domain) between BER and SNR in case of a Rayleigh fading channel. But, with the help of diversity techniques, this slope can be improved increasing the reliability of the data. We can conclude that diversity combining can lead to significant power savings.

A system whose BER curve has the same slope as MRC (but perhaps shifted to the right) with M-branches is said to exploit full M-branch diversity. Throughout this thesis, we consider a BPSK modulated system. The performance measure that we use is 'achievable diversity'. Hence, we compare the performance of our protocols (described in later chapters) with the MRC performance given in (3.4).

\section{Equal Gain Combining}

In equal gain combining technique, signals from all $M$ branches are weighted equally and then added. The performance of this technique is inferior to maximum ratio combining technique but is superior to selection diversity technique.

\subsection{Spatial Diversity and MIMO Communications}

The rapid growth of wireless multimedia services places demands on high-speed and high throughput requirements. From the last section, we know that diversity gain indicates how fast bit-error-rate (BER) decays with signal-to-noise ratio (SNR). Reducing the BER helps to satisfy high throughput and high speed requirements of advanced wireless communications. One way to decrease BER in the system is to transmit the signal with large transmit power and thus increasing the SNR at the receiver. But wireless communication is interference limited. In case of a system employing a CDMA technique, increasing the power of some of the users implies increasing the interference for the remaining users in the system. So, increasing the transmit power to reduce BER is not a good solution. Also, in a battery operated devices, the transmit power is limited. Spatial diversity is the most popular technique to achieve diversity gains without increasing the transmit power, transmission time and bandwidth. Spatial diversity can be achieved by implementing multiple antennas either at the transmitter (transmit diversity or multiple-input single-output communication) or at 
the receiver (receive diversity or single-input multiple-output communication) or both at transmitter and receiver (multiple-input multiple-output communication). It is well-known that multiple-input multiple-output (MIMO) communications have advantages of improving link reliability, and the capacity of wireless systems. By adding multiple antennas in the system, total diversity branches as seen at the receiver are increased, which increases the average SNR at the receiver. A system with $M$ transmit and $N$ receive antennas can provide a maximum diversity gain of $M N[16]$.

\subsubsection{Capacity of MIMO Channel}

Information-theoretic analysis shows that MIMO systems can offer significant capacity gains over single-input single-output (SISO) channels $[17,18,19]$. Here, we present important results for the capacity of MIMO systems.

Consider a MIMO system incorporating $M$ transmit and $N$ receive antennas. We assume block fading channel model where the channel is assumed to be constant over the period $T$ of one block of data and then changes independently after each block of $T$ symbol periods. The discrete time signal model for MIMO channnel in this case can be written as

$$
\boldsymbol{Y}=\sqrt{\frac{\mathrm{SNR}}{M}} \boldsymbol{H} \boldsymbol{X}+\boldsymbol{W}
$$

where $\boldsymbol{X} \in \mathcal{C}^{M \times T}$ is the transmitted signal matrix, $\boldsymbol{Y} \in \mathcal{C}^{N \times T}$ is the received signal matrix , $\boldsymbol{W} \sim \mathcal{N}_{c}(0, \boldsymbol{I})$ is AWGN noise and the coefficients $h_{i j}$ of the channel matrix $\boldsymbol{H}$ are independent, complex Gaussian random variables with zero mean and unit variance. SNR is the average signal-to-noise ratio at each receiving antenna. We assume that the channel state information (CSI) is available at the receiver but not at the transmitter. In practice, channel matrix $\boldsymbol{H}$ can be estimated using training sequence. Then the average or ergodic channel capacity is given by

$$
C=E\left[\log _{2} \operatorname{det}\left(I_{N}+\frac{\mathrm{SNR}}{M} \boldsymbol{H} \boldsymbol{H}^{\mathrm{H}}\right)\right] \mathrm{bits} / \mathrm{sec} / \mathrm{Hz}
$$

which can also be written as [16], 


$$
C=E \sum_{i=1}^{r} \log _{2} \operatorname{det}\left(I_{N}+\frac{\mathrm{SNR}}{M} \lambda_{i}\right)
$$

where $r$ is the rank of $\boldsymbol{H}$ and $\lambda_{i}$ are the positive eigenvalues of $\boldsymbol{H} \boldsymbol{H}^{\mathrm{H}}$. The equation (3.8) represents the capacity of MIMO channel as the sum of the capacities of $r$ SISO channels with channel gains $\lambda_{i}$. Also, at high SNRs, the channel capacity increases with SNR as $K \log \mathrm{SNR}$ in contrast to $\log \mathrm{SNR}$ for SISO channels [20], where $K=\min \{M, N\}$.

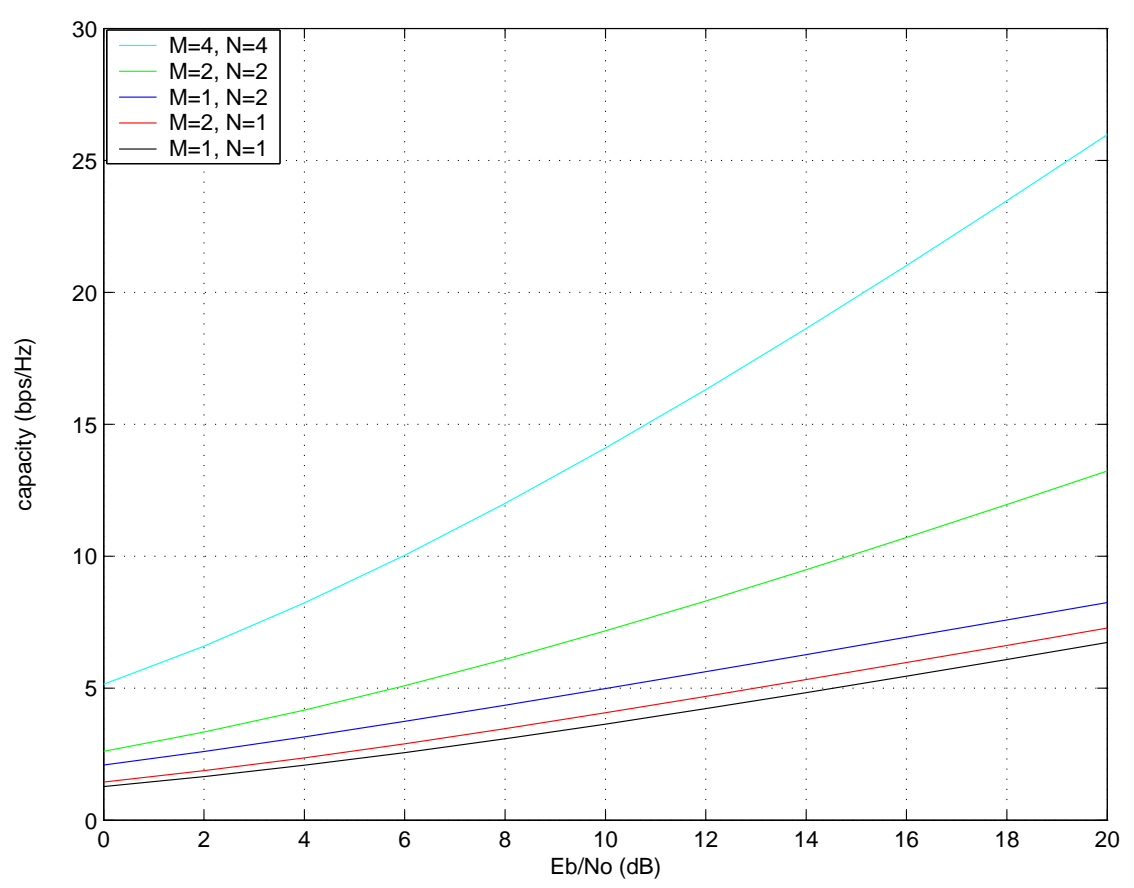

Figure 3.2: Ergodic capacity of MIMO system with different antenna configurations.

Fig. 3.2 gives the average-capacity curves as a function of SNR for different MIMO antenna configurations. We note that the capacity of SIMO channel is higher than MISO channel. It is also seen that, capacity increases linearly with $\min \{M, N\}$, when both number of transmit antennas $(M)$ and receive antennas $(N)$ increase. But if the number of receive antennas is fixed and the number of transmit antennas increases, the capacity saturates at a fixed value while if number of transmit antennas is fixed and the number of receive antennas increases, the capacity increases logarithmically with $N$. All the above channel capacity calculations are based on the assumption that the paths between multiple transmit 
and receive antennas undergo uncorrelated fading. When there exists correlation between individual fading coefficients, the capacity of the MIMO system decreases. It is seen that urban and indoor environments with rich scattering provide much higher MIMO capacities than rural environments. Also, we assumed that CSI is present only at the receiver. But if the channel is also known at the transmitter, some capacity gains can be obtained at low SNRs [21]. In the following sections, we will discuss two well-known MIMO techniques, one for achieving high data rates (or multiplexing gains) and another for realizing high diversity gains.

\subsubsection{The Diversity-Multiplexing Tradeoff}

MIMO systems offer advantages over single-input single-output (SISO) channels, in terms of diversity gains (which improve reliability of data), multiplexing gains (which improve spectral efficiency) and the interference reduction capability (which improves energy efficiency). But it is not possible to exploit all of the gains of MIMO systems simultaneously. Maximizing one type of gain may not maximize another gain simultaneously. There exists an optimal diversity-multiplexing tradeoff which sets a fundamental performance limit of a MIMO channel system [4]. The diversity-multiplexing tradeoff is essentially the tradeoff between bit-error-rate and the data rate in a channel. We consider a $M \times N$ MIMO block fading channel with $T$ as the block length. It has been shown that if $T>M+N-1$, the optimal diversity gain $d(r)$ achievable by any coding scheme of block length $T$ and multiplexing gain $r$ (bits/sec/Hz) ${ }^{1}$ is $(M-r)(N-r)$. This can be interpreted as out of total $M$ transmit and $N$ receive antennas, $M-r$ transmit and $N-r$ receive antennas are being used to exploit diversity and the remaining $r$ transmit and receive antennas are being used to exploit multiplexing gain. Hence the spatial multiplexing gain can be increased by one by adding one transmit and one receive antenna while retaining the same diversity gain [20].

\footnotetext{
${ }^{1} \mathrm{~A}$ scheme achieves spatial multiplexing gain of $r$ if the supported data rate $R(\mathrm{bits} / \mathrm{sec}) \approx r \log \mathrm{SNR}$
} 


\subsubsection{The BLAST System}

As we saw in the previous section, a system employing MIMO communication structure can exploit diversity gain or multiplexing gain but not both. Bell Labs Space Time Architecture (BLAST) was first proposed by Foschini [22] for high data rate communications. In this method, independent symbol streams are transmitted from each transmit antenna. The receiver incorporating multiple receive antennas then processes these symbol streams using appropriate detection algorithm. As independent symbol streams are transmitted from multiple antennas simultaneously, the spectral efficiency ( or data rate ) of this scheme is high.

Let us look at the signal model. Consider a system with $M$ transmit antennas and $N$ receive antennas. Then the received signal during one channel use can be represented by

$$
\underbrace{\left[\begin{array}{c}
r_{1} \\
r_{2} \\
\vdots \\
r_{N}
\end{array}\right]}_{\boldsymbol{r}}=\underbrace{\left[\begin{array}{cccc}
h^{(1,1)} & h^{(1,2)} & \ldots & h^{(1, M)} \\
h^{(2,1)} & h^{(2,2)} & \ldots & h^{(2, M)} \\
\vdots & \vdots & \vdots & \vdots \\
h^{(N, 1)} & h^{(N, 2)} & \ldots & h^{(N, M)}
\end{array}\right]}_{\boldsymbol{H}} \underbrace{\left[\begin{array}{c}
x_{1} \\
x_{2} \\
\vdots \\
x_{M}
\end{array}\right]}_{\boldsymbol{x}}+\underbrace{\left[\begin{array}{c}
n_{1} \\
n_{2} \\
\vdots \\
n_{N}
\end{array}\right]}_{\boldsymbol{n}}
$$

where $\boldsymbol{n} \sim \mathcal{N}_{c}\left(0, \sigma^{2} \boldsymbol{I}\right)$. ML is optimal, in terms of the error rate. The ML decision rule assuming receiver CSI, is given by

$$
\hat{\boldsymbol{x}}=\arg \min _{\boldsymbol{x}}\|\boldsymbol{r}-\boldsymbol{H} \boldsymbol{x}\|^{2} .
$$

Thus an exhaustive search is performed over all possible transmitted signal vectors $\boldsymbol{x}$. Though ML algorithm is optimal, its computational complexity is too high. There exist other sub-optimal detection techniques such as successive nulling/cancellation and zero forcing, which are comparatively less complex [23].

\subsubsection{Space-Time Coding}

Space time coding techniques target on achieving high diversity gains. There are some basic differences between BLAST and space-time coding (STC) [21]. 
- The goal of the BLAST system is to achieve multiplexing gain. BLAST system transmits $M$ symbols/channel use to achieve this goal. Whereas STC tries to achieve maximum diversity gain. Thus STC transmits 1 reliable symbol/channel use. The spectral efficiency of STC schemes can be improved with the help of higher constellations but at the expense of degraded error performance.

- The optimal receiver for BLAST as given in (3.10) is very complex and the performance of suboptimal receivers such as the nulling/cancellation detection is limited by error propagation mechanism. The optimal receiver for most of the space time coding techniques as we will see is linear in nature (only in synchronous environments) and hence offers a very low complexity.

- BLAST requires $N \geq M$, that may not be possible when a receiver is also a mobile unit or a battery operated device. There is no such problem posed in case of STC techniques.

In the space time coding technique [24, 25, 26, 27], the same information is transmitted from different antennas in an appropriate manner to improve the reliability of the system.

Space time block coding (STBC) and space time trellis coding (STTC) are the two main types of space time coding techniques. Space time trellis codes achieve coding gain in addition to the diversity gain but the decoding complexity of trellis codes is much higher as compared to space time block codes.

Figs. 3.3, 3.4, compare the STBC scheme and BLAST scheme for 2:2 MIMO system via their BER and throughput simulations. The throughput is the number of successfully received information bits per transmission. The normalized throughput, $T_{h}$ is calculated here as

$$
T_{h}=R(1-\mathrm{BER})^{M} \text { bits/time slot }
$$

where $R$ is the normalized rate and $M$ is the packet size. As discussed before, BER performance shows that the diversity gain of Alamouti space time code (or any orthogonal code) is high as compared to BLAST scheme. As can be seen, throughput performance of STBC scheme is better than BLAST at low SNRs but at high SNRs, BLAST scheme 


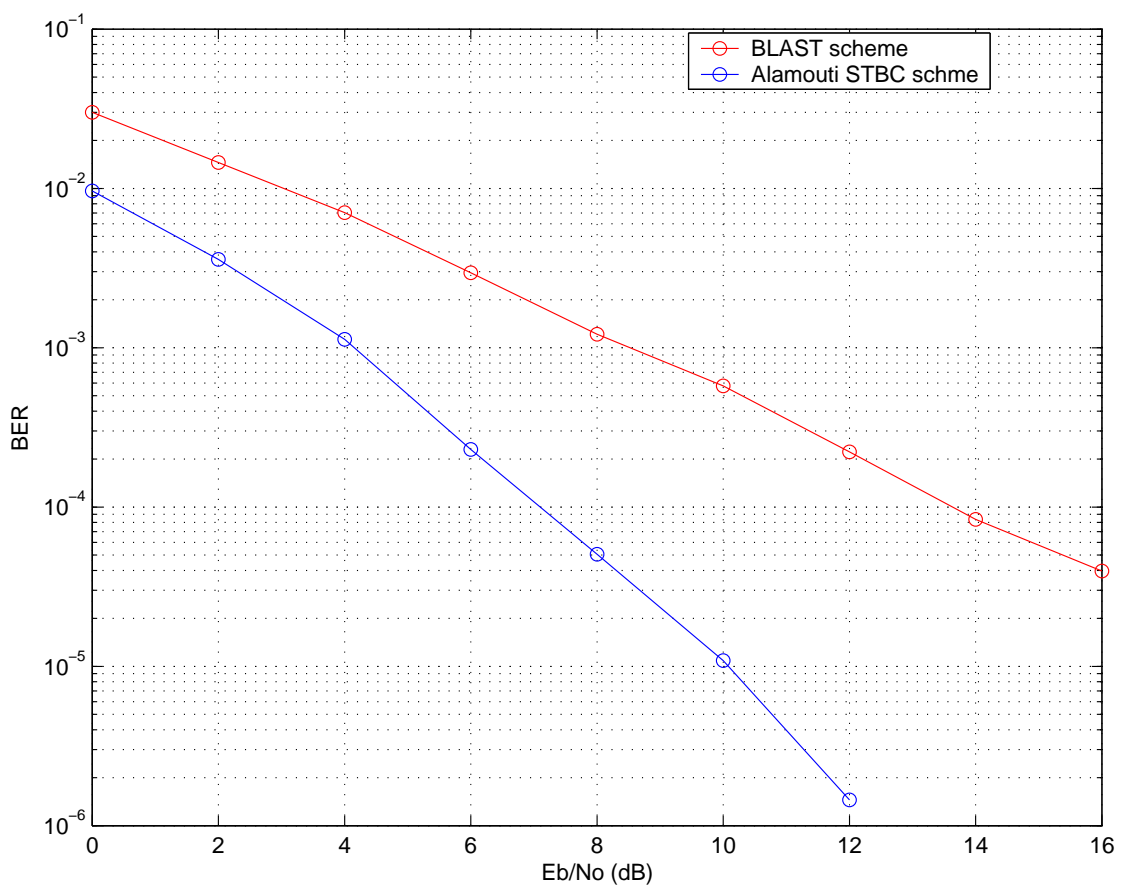

Figure 3.3: BER performance comparison between BLAST and STBC schemes with BPSK modulation for 2:2 MIMO system. ML detection is used.

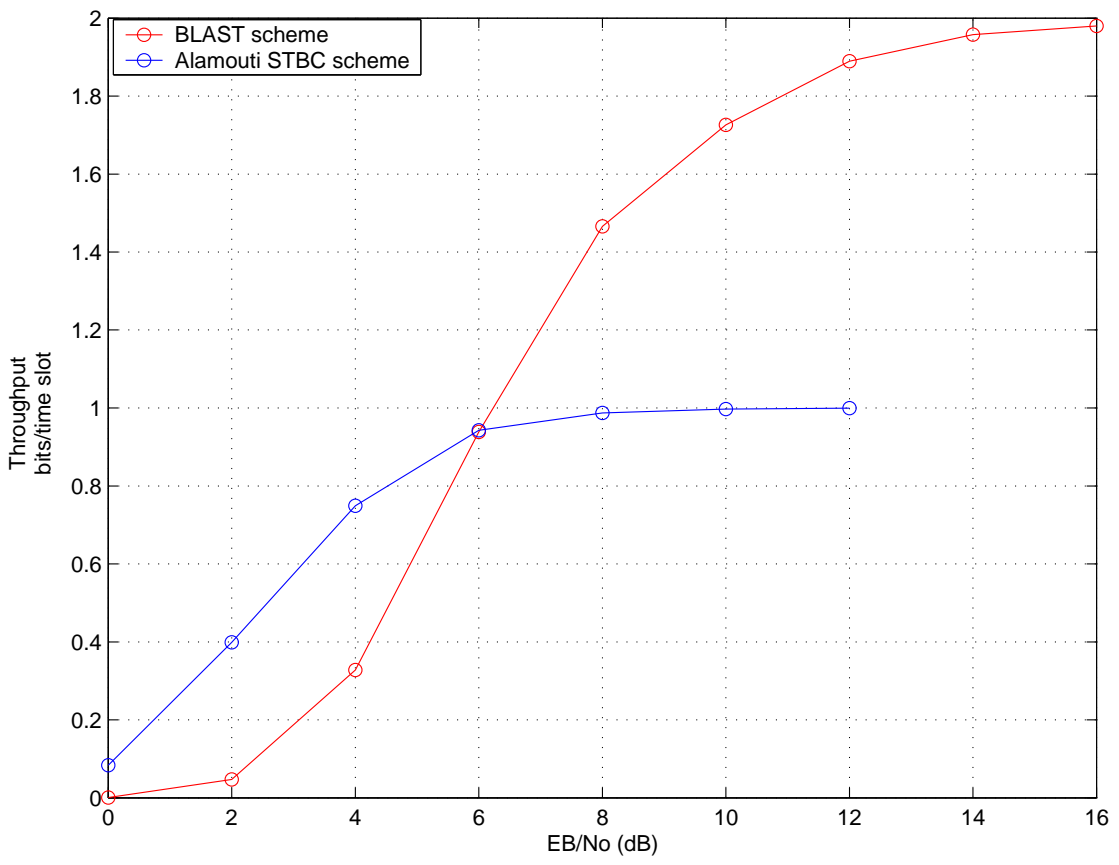

Figure 3.4: Throuput performance comparison between BLAST and STBC schemes with BPSK modulation for 2:2 MIMO system. The packet size is 256. ML detection is performed. 
offers better throughput. For the same reason, BLAST technique is more often used in high SNR regimes. Note that, in case of a BLAST technique with two transmit antennas, the normalized throughput is limited to 2 (because the rate $R$ is 2 bits/transmission), while in Alamouti's space time coding technique, it is limited to 1.

In this thesis, we will be using popular Alamouti's space time block code (STBC) [24] with BPSK modulation. The Alamouti STBC offers diversity gains with simple ML decoding. It uses two transmit antennas, number of receive antennas may vary and does not affect the code construction. Let's consider a MISO system with two transmit antennas and one receive antenna employing STBC scheme. Alamouti's space time code block can be represented by

$$
\left[\begin{array}{cc}
x_{1} & x_{2} \\
-x_{2}^{*} & x_{1}^{*}
\end{array}\right]
$$

where the symbols from the first row of the above matrix are transmitted from two transmit antennas during the first time slot and symbols from second row are transmitted during the second time slot. The matched filtered output at the receive antenna during first time slot (assuming synchronous communication) is

$$
r_{1}=h_{1} x_{1}+h_{2} x_{2}+n_{1}
$$

and that in the second time slot is

$$
r_{2}=-h_{1} x_{2}^{*}+h_{2} x_{1}^{*}+n_{2}
$$

Then the discrete time signal model can be written as

$$
\underbrace{\left[\begin{array}{c}
r_{1} \\
r_{2}^{*}
\end{array}\right]}_{\boldsymbol{r}}=\underbrace{\left[\begin{array}{cc}
h_{1} & h_{2} \\
h_{2}^{*} & -h_{1}^{*}
\end{array}\right]}_{\boldsymbol{H}} \underbrace{\left[\begin{array}{c}
x_{1} \\
x_{2}
\end{array}\right]}_{\boldsymbol{x}}+\underbrace{\left[\begin{array}{c}
n_{1} \\
n_{2}^{*}
\end{array}\right]}_{\boldsymbol{n}}
$$

where $h_{i}$ is the channel gain between transmit antenna $i$ and a receive antenna and $\boldsymbol{n} \sim$ $\mathcal{N}_{c}\left(0, \sigma^{2} \boldsymbol{I}\right)$. At the receiver, we form the decision statistics as [15]

$$
\begin{aligned}
\boldsymbol{d} & =\boldsymbol{H}^{H} \boldsymbol{r} \\
& =\boldsymbol{H}^{H} \boldsymbol{H} \boldsymbol{x}+\boldsymbol{H}^{H} \boldsymbol{n} \\
& =\left[\begin{array}{cc}
E_{h} & 0 \\
0 & E_{h}
\end{array}\right]+\boldsymbol{v}
\end{aligned}
$$


where $E_{h}=\left|h_{1}\right|^{2}+\left|h_{2}\right|^{2}$. We observe that since $\boldsymbol{H}^{H} \boldsymbol{H}$ is diagonal, there is no intersymbol interference. The bit estimates are $\hat{\boldsymbol{x}}=\operatorname{sign}\{\Re[\boldsymbol{d}]\}$, assuming BPSK modulation. These are nothing but the ML estimates. The diversity gain of STBC scheme is same as that of twobranch MRC (MRC-2) but there exists a coding loss of $3 \mathrm{~dB}$ because we actually transmit $\boldsymbol{x}=\frac{1}{\sqrt{2}}\left[\begin{array}{ll}x_{1} & x_{2}\end{array}\right]$ to satisfy the power constraints. Since two symbols are transmitted over two time slots, the rate of this scheme is 1 . This scheme is useful when high throughput is required at low SNR. Alamouti's scheme works for only two transmit antenna case. This can be generalized to any number of transmit antennas [25].

\subsection{Cooperative Diversity}

It is seen that MIMO communications offer various advantages including high capacity and diversity gains. To achieve these advantages, the channel coefficients along the paths between multiple transmit and receive antennas should be uncorrelated. This requires the antenna spacing of about several wavelengths of the carrier frequency. But in many cases, it is not possible to implement multiple antennas at the mobile units with the desired spacing between them.
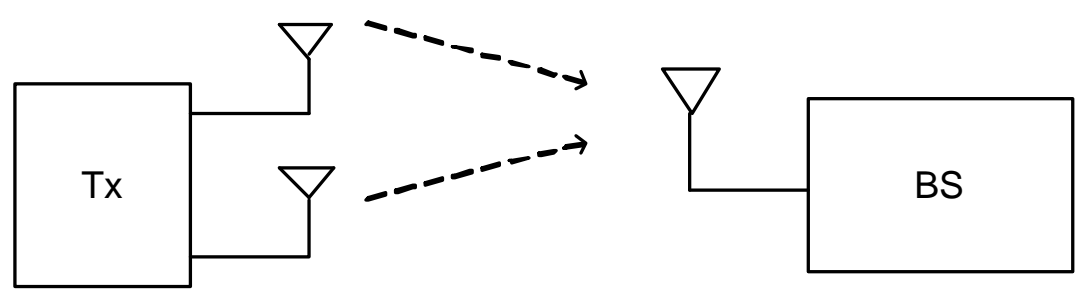

Figure 3.5: Conventional multi-antenna system array where multiple antennas are implemented on a single unit and are physically connected.

In that case, spatial diversity can then be achieved via the cooperation of single antenna mobile units in the system. These units form a virtual array instead of a physical array as in conventional MIMO systems. This technique which eliminates the need for incorporating multiple antennas on a single mobile units via the cooperation among multiple users is called 'cooperative diversity'. Fig. 3.5 shows a conventional multi-antenna system which consists of physical antenna array. Here, a single device incorporates multiple antennas 


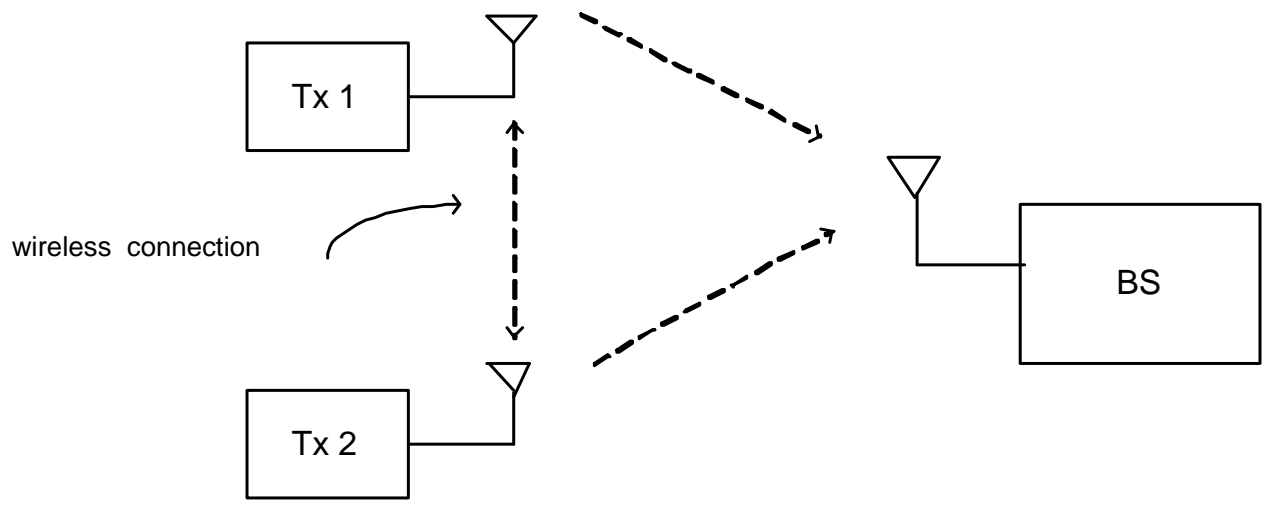

Figure 3.6: Distributed multi-antenna system where individual antennas are implemented on a separate mobile unit. There is no physical connection between multiple antennas in this case.

which are connected by a fixed backbone network. In contrast, Fig. 3.6 indicates a multiantenna system where instead of implementing multiple antennas on a single device, a virtual antenna array is formed using individual devices which talk to each other wirelessly and then cooperatively send data to the desired destination.

The most existing strategies for achieving cooperative diversity can be placed into one of two categories, Decode and Forward (DF), and Amplify and Forward (AF).

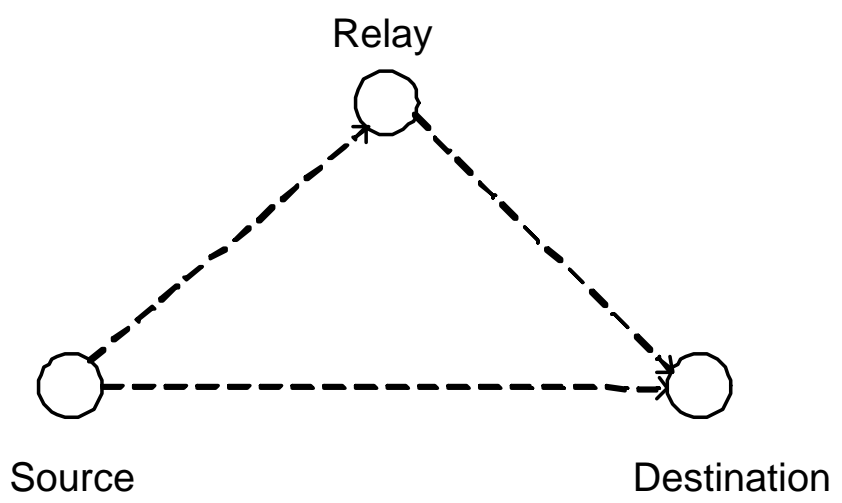

Figure 3.7: A simple relay case

We will first consider a simple relay problem to illustrate the DF and AF protocols. The concept of relay channels can then be applied to user cooperation. Fig. 3.7 shows a system with three users. Suppose the source wants to transmit data to a destination. In a noncooperative mode, it will simply send the data towards the destination and destination would 
try to decode that data. Estimates at the destination will depend upon the instantaneous fading channel coefficient. But if we let the same data to be forwarded over a different independent channel, the diversity principle will enable the reduction in the error-rate at the destination via use of the independent copy of the same data. Suppose now, the relay terminal helps the source forward its data. Relay terminals in the network may be viewed as virtual antennas for the nodes they serve. Whenever source transmits data, the relay terminals in the vicinity of the source also receive this transmission because of the broadcast nature of the wireless medium. There are two categories of methods by which relay terminal can help the source. ( Both methods consist of two transmission phases. In the first phase, the source broadcasts to its destination and all relays. During the second phase, the other terminals relay to the destination). In the first category of methods, the relay terminal tries to decode the data received over a fading channel. Upon successful decoding, it again sends the same information over another independent channel to the destination. Now the destination has two independent copies of the same signal and hence the detector at the destination terminal can output better estimates. Since the decoding operation is performed at the relay node, this is termed as 'decode and forward' mode of operation. In another category of methods, the relay terminal observes a transmission from the source. The data available at the relay is corrupted due to the source-relay fading channel. Instead of decoding this data, the relay terminal just forwards the received signal to the destination, possibly after amplifying it. This mode of operation is called as 'amplify and forward' mode. There are various advantages and disadvantages of both modes of operation. In AF case, the relay terminal simply retransmits the signal received from the source terminal without any decoding and hence requires significantly lower computational complexity and less delay (because it doesn't need to wait for a full codeword before re-transmission) when compared with the DF protocol. But since relay terminals in the AF protocol forward the corrupted copy of the signal, the bit-error-rate performance of AF protocol is worse when compared to DF protocol. The relay concept can be extended to user cooperation where cooperating units have their own data also to transmit as against the relay case. The concept of user cooperation in a cellular uplink was first popularized by Sendonaris et el.[5, 6]. Later in this thesis, we also propose multi-user cooperation schemes for the cellular uplink. 
The cooperative protocols can further be categorized into static and adaptive (dynamic) protocols. Adaptive protocols can be implemented by allowing feedback between cooperating nodes. The helping nodes in an adaptive protocol change the protocol depending on CSI, while in case of static protocols, they sometimes forward and sometimes don't depending upon whether decoding was possible at the relay, but the protocol remains the same.

The concept of cooperative diversity can be applied to ad-hoc networks or cellular networks. Implementation of cooperative diversity protocols requires cross-layer network design since it involves the design of medium access control protocols and routing strategies. Most of the research on cooperative diversity considers relaying schemes and its applications to wireless ad-hoc networks $[28,29,30,31,32,33,34]$. Cooperative diversity for the cellular networks has been studied in $[2,5,6,35,36,37,38]$. In the next two chapters, we discuss new two-user and three-or-more users sharing protocols achieving cooperative diversity.

\subsection{Summary}

Diversity techniques play an important role in the wireless fading environments. Among the many diversity techniques available, MIMO systems have attracted more attention. MIMO systems provide diversity gains and capacity gains over SISO channel at the same transmit power and bandwidth requirements. STC and BLAST are the two powerful MIMO techniques. STC provides diversity gains by improving the reliability of the wireless links while BLAST offers significant improvement in the multiplexing gains. There exists a fundamental trade-off between diversity gain and multiplexing gain. Whenever it is not possible to accommodate multiple antennas on a single device, spatial diversity can be achieved via cooperation of single antenna units. The most existing sharing strategies can be placed into one of two categories, AF or DF. Both AF and DF have advantages and disadvantages of their own. 


\section{Chapter 4}

\section{Multi-User DF Sharing Schemes with Perfect Inter-User Channels}

There has been a growing research activity over the past decade on the design issues of ad hoc wireless networks. Ad hoc wireless networks are the communication networks without any centralized control. That is, mobile nodes self-configure to form a network without any established infrastructure. The communication between various nodes within the network takes place via multihop routing. They find applications in military communications, home networking, and sensor networks [39]. It is believed that implementation of ad hoc networks may lead to significant energy savings over the conventional networks incorporating single hop routing. An idea of multihop routing can be applied to cellular networks particularly to the cellular uplink. In a conventional cellular telephone networks, the coverage zone is divided into small cells. A mobile terminal communicates directly with the base station associated with that particular cell. Thus all the communication is done via the base station through single hop routing and there is no peer to peer communication between mobile units. The drawback of single hop routing relative to multihop routing in cellular networks is that more transmit power is required to reach far distances which can cause interference to other users in the system. To serve more connections in a crowded area, number of base stations will have to be increased. But this may lead to high costs for deployment of base stations. Also, in the future, same subscribers will have a much higher demand on the transmission rates. But subscribers will not be willing to pay more, so a drastic increase in number of 
base stations seems impracticable. To overcome some of these difficulties and to improve the communication performance of the link, multiple antenna techniques may be employed. As discussed in Chapter 1, to obtain full benefits of MIMO systems, it is necessary to have independent fading channel gains between multiple paths created by multiple antennas. This is possible if the antenna spacing is at least half a wavelength of the transmitted signal. However, for $3 \mathrm{G}$ wireless systems using carrier frequencies of the order of $1 \mathrm{GHz}$, wavelength can not be much smaller than $0.3 \mathrm{~m}$. So, it is not possible to implement multiple antennas (with the desired spacing) at the mobile units operating in the cellular uplink due to size and power consumption constraints. In the last chapter, we saw that the benefits of the spatial diversity can also be achieved through the cooperation of distributed users in the system. Recently, it has been shown that multihop routing to the base station can improve the system performance dramatically [40]. Using multihop routing, data packets can be forwarded from mobile units to the base station via intermediate mobile units. The cooperation between mobile units achieves better link reliability.

Recently, some work has been done on the cooperative diversity techniques for wireless networks. In $[2,37]$, the authors develop performance characterizations in terms of outage events and associated outage probabilities for half duplex cooperative diversity protocols. The authors do not specify any modulation or coding structure. Their work provides a performance benchmark assuming optimal codes. In [3, 4], the authors provide the diversitymultiplexing tradeoff for cooperative half duplex channels and MIMO channels in general. None of these works presents specific modulations, or receiver designs, and they do not investigate performance in practical environments. In $[5,6]$, the authors develop two user cooperation protocols for a CDMA system and characterize the analytical performance under the assumptions of orthogonal spreading codes, and synchronous transmission. The protocol given here is also not generalizable to more than two cooperating users and it can not operate at full rate. Also, most of the work in the literature [3, 37, 31, 32] assumes either half-duplex communication or multi-channel environments in which parallel transmissions can be carried out without interference. As against this, we assume full-duplex communication for the reasons explained in the next section.

In this chapter, we propose and compare new multi-user sharing schemes and practical 
receiver design for the cooperative diversity in cellular uplink. We use BPSK modulation in our simulations. Specifically we consider two-user and three-or-more user cooperation schemes. The data sharing schemes simultaneously provide full diversity, as measured by the BER slope, and high throughput, as compared to the Sendonaris scheme [5, 6] and to a conventional no-sharing uplink. Our sharing schemes operate at full rate but suffer from multiple-access interference. Our receivers are restricted to linear designs that jointly suppress multiuser interference and perform space-time decoding. They have the advantages of low complexity and they are easy to make adaptive. We consider both synchronous and asynchronous communication scenarios. We focus on a CDMA system ${ }^{1}$ though the same idea can be applied to time or frequency division multiplexing with little change.

\subsection{System Model}

Our BPSK-modulated CDMA uplink consists of mobile units that are assigned to cooperating groups by a higher-layer protocol. For example, the network terminals can perform some threshold tests. The terminals having good link SINR (signal-to-noise-plusinterference-ratio) between each other can form one group. We do not consider the problem of optimal group assignment in this thesis. But we note that there exists a practical upper limit on group-size or cluster size. If the cluster has too many nodes, the mobile units will expend much of their energy receiving transmissions from other units. Hence the limit on number of users per cluster depends mostly on the ratio of the energy consumed while receiving a signal and the energy required to transmit it [42]. All mobile units including the base station are equipped with a single antenna though the base station can employ multiple antennas to improve the performance. Also, each user is assigned a single spreading code. We assume full-duplex communication, that is, cooperating users can transmit and receive simultaneously on the same frequency. Authors in $[31,32]$ consider "cheap" relay networks which operate in time-division multiplexing (TDD) mode and hence preclude terminals from full-duplex communication. TDD or half-duplex techniques divide the radio channel into

\footnotetext{
${ }^{1}$ Though, three multiple access schemes are theoretically equivalent in AWGN channels, there are notable differences when multipath fading channel is considered [41].
} 
time slots to allow transmission of data during part of the time period and signal reception in the remainder of the time period. The key motivation for our assumption on full-duplex communication comes from the fact that the full-duplex systems have bandwidth efficiency superior to half-duplex systems. Also, even though a terminal's transmitted signal will have a detrimental effect on the received signals of other terminals in case of full-duplex systems, this effect can be cancelled if the terminals know the relevant antenna gains [5]. Since current radios operate in TDD mode when transmitting and receiving frequencies are the same, the design of RF radios that are able to send and receive simultaneously in the same frequency band will of course require expensive and precise transmit-receive circuitry. Use of spreading codes or co-located antennas to create separate channels may also be useful to isolate the transmitted signal from the received signal [5]. Therefore, the assumption on full-duplex communication may not be a realistic assumption for 'low-cost' networks but is certainly not an impractical premise.

In this chapter, we assume that the users are grouped together such that the interuser channels have high SNR, i.e., we do not consider the effects of imperfect inter-user channels. In the next chapter, we will deal with more practical, noisy inter-user channels. For simplicity and to produce baseline results, we have assumed a system without forward errorcorrection coding (FEC), so that the DF protocols discussed here are actually implemented as demodulate-and-forward protocols, but a FEC outer code can easily be added to the system. In case of DF protocols, after detection, cooperating users transmit their estimated symbols in a simultaneous fashion where symbol synchronization is not strictly observed. We assume asynchronous communications. We provide simulation results for synchronous communication for comparison purposes. We use zero padding between successive blocks of data to avoid inter-block-interference ${ }^{2}$. Note that zero padding reduces the effective rate of the system. Zero padding can be avoided but at the cost of little loss in coding gain. The spreading codes provide processing gain $N$ and, in contrast to $[5,6]$, they are not assumed orthogonal. The AWGN noise power is $\sigma^{2}$ and we assume perfect power control so that the Rayleigh flat-fading channels between users and the base station are assumed

\footnotetext{
${ }^{2}$ Here by inter-block interference we mean, the interference caused by individual blocks of data transmitted from different mobile units
} 
statistically identical. We do not consider the important problem of power control in the context of data sharing. But we note that power control can be used to reduce the transmit power while achieving the required data rate and error probabilities. Power control strategies can increase the power and rate when channel conditions are good and decrease the same under poor channel conditions. Channel state information is assumed to be available at the base station, where linear joint MMSE multiuser detection and space-time decoding is performed. Also, the channel matrix is assumed to be constant over a block of data which changes independently from one block to the next block.

In the next sections, we consider two-user and three-or-more user sharing protocols.

\subsection{Two-User Decode and Forward Cooperation}

Here we consider a $K$-user system where the users have been grouped into pairs for uplink transmission. Each user has information to transmit to the base station. In each time slot, each user transmits its own data on its spreading code plus its partner's previous data on that user's spreading code. We assume that the partner's data has been estimated perfectly (for the simulations in this chapter) from signals received in the previous time slot. Thus, symbols transmitted from two cooperating users during the $n$-th time slot are

$$
\begin{array}{ll}
\text { User 1: } & b_{1}[n] s_{1}+b_{2}[n-1] s_{2} \\
\text { User 2: } & b_{2}[n] s_{2}+b_{1}[n-1] s_{1}
\end{array}
$$

where, $b_{j}[i]$ is the symbol transmitted from user $j$ at time slot $i$ and $s_{k}$ is the signature waveform of user $k$. For the received signal model described in the next section, we assume that one frame consists of two symbols from each user (transmitted over three time slots). Other frame sizes can be used with little change in performance.

Note that in contrast to the protocols in [3] and [5], the proposed two-user protocol can operate at full rate, assuming full duplex, i.e., users can transmit and receive simultaneously. 


\subsection{Three-or-More User Decode and Forward Cooper- ation}

The use of space time codes for cooperative transmission was first proposed in [37]. Here, we develop a specific protocol for three or more users that makes use of space-time block codes $[24,25]$. As an example, we consider a $K$ user system whose users have been assigned to groups of three. Similar to the two-user protocol, each user sends its own new data in every time slot. Simultaneously, each user transmits the other users' previous data using a distributed space-time block code. In particular, user 1 will form a space time code with user 2 to send user 3's previous data and user 1 will also form a space time code with user 3 to send user 2's previous data. For three-user sharing, we make use of the popular Alamouti space time code in our simulations which has been adopted in a number of $3 \mathrm{G}$ WCDMA standards. Note that orthogonal space-time block codes cannot provide full diversity in asynchronous environments for some delay profiles e.g., if the relative uplink delay between users is exactly one symbol interval. When the relative delay between the signals arriving from two distinct nodes is exactly one symbol interval, the loss in rank of a standard Alamouti scheme is easily observed [43]. The Alamouti code or the orthogonal standard space-time codes [25] demonstrate no diversity in such environments. To deal with this problem, 'macroscopic space time codes' have been designed which provide full diversity even if the relative delay is exactly one symbol interval [43]. The macroscopic space-time codes developed in [43] relax a requirement on symbol synchronization at the receiver and thus can greatly simplify the system medium access control (MAC) requirements. The authors in [43] propose the construction of a full-rank macroscopic space-time codes over the delay set $\left\{-L T_{s},(-L+1) T_{s}, \ldots-T_{s}, 0, T_{s}, \ldots L T_{s}\right\}$ where the code length $2 N$ is equal to four times the least common multiple of $1,2, \ldots, L$. The proposed code construction is as follows,

$$
\left(\begin{array}{ccccccccc}
s_{1} & s_{2} & \ldots & s_{N+1} & s_{N} & s_{N+1} & \ldots & s_{2 N-1} & s_{2 N} \\
-s_{N+1}^{*} & -s_{N+2}^{*} & \ldots & -s_{2 N-1}^{*} & -s_{2 N}^{*} & s_{1} & \ldots & s_{N-1} & s_{N}
\end{array}\right)
$$

where $\left\{s_{i}\right\}$ are the independent BPSK symbols. Similar to the Alamouti code, the first row of the above code matrix is transmitted from the first transmit antenna and the second row is 
transmitted from the second transmit antenna. An example of BPSK orthogonal macroscopic space-time code that maintains full rank for each relative delay in the set $\left\{-T_{s}, 0, T_{s}\right\}$ is

$$
\left(\begin{array}{cccc}
s_{0} & s_{1} & s_{2} & s_{3} \\
-s_{2} & s_{3} & s_{0} & -s_{1}
\end{array}\right)
$$

We make use of the code construction in (4.2) when we compare the performance of macroscopic space-time code and Alamouti's space-time code.

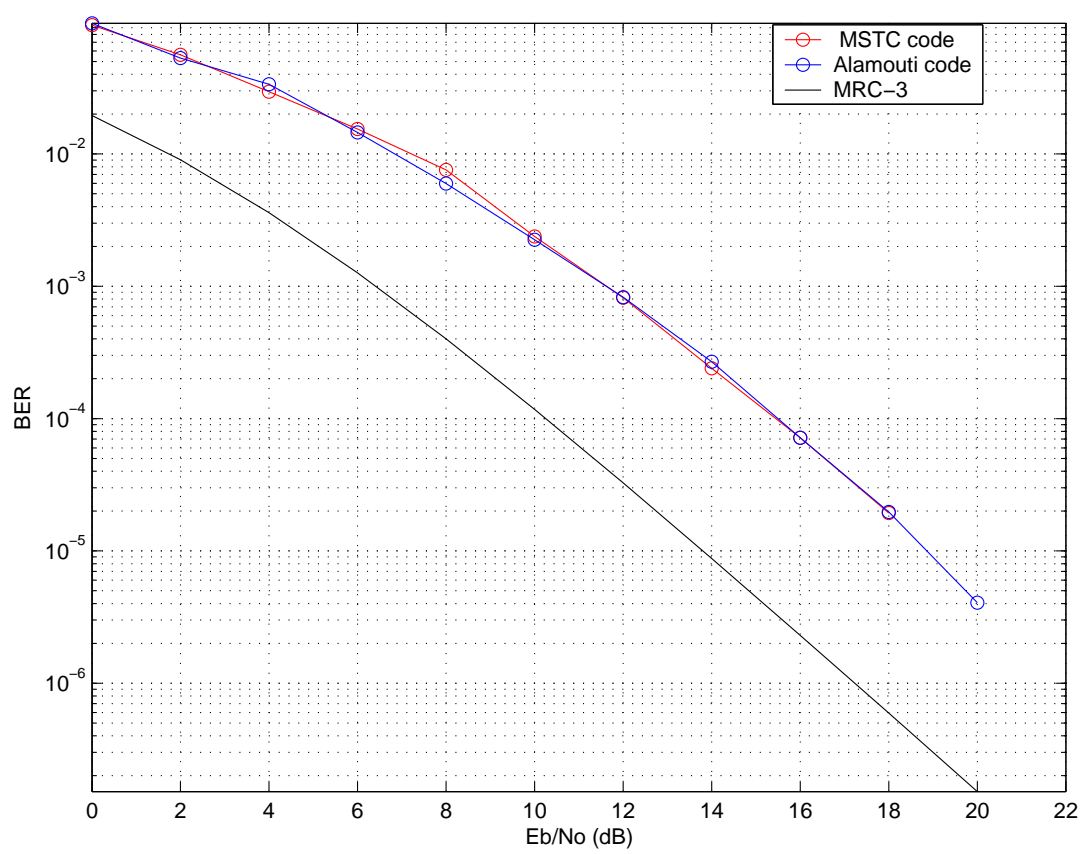

Figure 4.1: Bit-error-rate performance of three-user sharing scheme in asynchronous uplink environment when delays are uniformly distributed in the range $\left[0, T_{s}\right]$. Performance of maximum-ratio-combining is also included for comparison.

Figs. 4.1 and 4.2 illustrate the effect of different delay profiles on the diversity gain. It can be seen that when the delays are continuous in the range $\left[0, T_{s}\right]$, Alamouti code or in general orthogonal space-time design and macroscopic space-time code have the same performance. But when relative delay between two antennas is exactly equal to one symbol interval, macroscopic space-time code shows significant performance improvement (in terms of the diversity gain) over orthogonal designs.

But, the length of the macroscopic space time code increases with the delay range. This complicates the receiver design. Also delay profiles in which relative uplink delay between users is exactly one symbol interval occur with probability zero, assuming delays are 


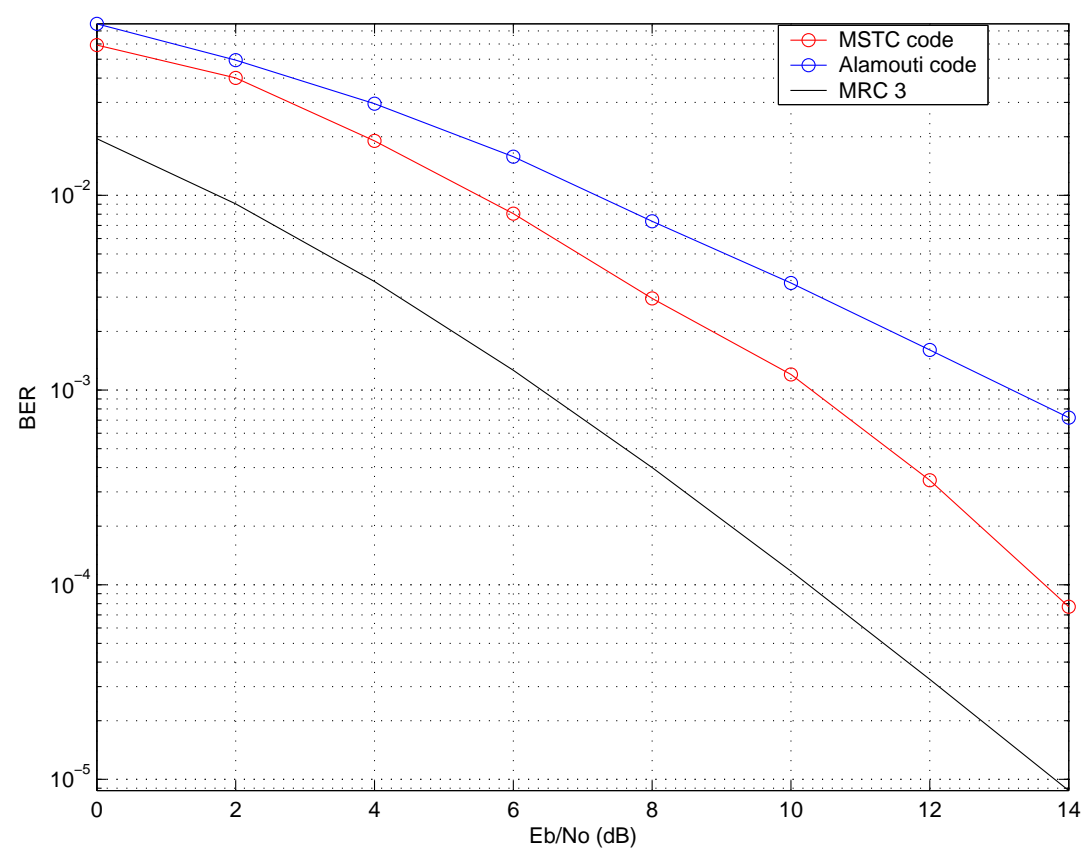

Figure 4.2: Bit-error-rate performance of three-user sharing scheme in asynchronous uplink environment when relative delay ie exactly one symbol interval. Performance of maximumratio-combining is also included for comparison.

uniformly distributed. In situations, where delays are continuous and uniformly distributed in the range $\left[0, T_{s}\right]$, performance of macroscopic space time code is approximately the same as Alamouti space time code. Taking into account these facts, we use Alamouti space-time code even in asynchronous communication environments. The data sharing scheme can be easily extended to more than two users using orthogonal/non-orthogonal designs. Full rate version of space time codes based on complex-orthogonal design exists only for two transmit antenna configuration. Other orthogonal designs for complex constellation exist but at lower rates [25]. Recently, non-orthogonal codes have been designed which lead to limited interference and which operate at full rate [44]. Since we are interested in the performance of a system in asynchronous communication environments, even orthogonal space time codes will lose their orthogonality. Hence, we can use full rate non-orthogonal space time code design in our system which are available for any transmit and receive antenna configurations.

It has been believed that cooperation schemes particularly those using distributed spacetime codes require coordination and negotiation among helping nodes to exploit diversity which adds extra cost to radio transmission. Since, in our system model, we assume asyn- 


\begin{tabular}{|l|l|l|l|}
\hline Time Slot & User1 & User 2 & User 3 \\
\hline \hline 1 & $b_{1}[1]$ & $b_{2}[1]$ & $b_{3}[1]$ \\
\hline 2 & $b_{1}[2]$ & $b_{2}[2]$ & $b_{3}[2]$ \\
\hline 3 & $b_{2}[1], b_{3}[1], b_{1}[3]$ & $b_{1}[1], b_{3}[2], b_{2}[3]$ & $b_{1}[2], b_{2}[2], b_{3}[3]$ \\
\hline 4 & $-b_{2}[2],-b_{3}[2], b_{1}[4]$ & $-b_{1}[2], b_{3}[1], b_{2}[4]$ & $b_{2}[1], b_{1}[1], b_{3}[4]$ \\
\hline 5 & $b_{2}[3], b_{3}[3]$ & $b_{1}[3], b_{3}[4]$ & $b_{1}[4], b_{2}[4]$ \\
\hline 6 & $-b_{2}[4],-b_{3}[4]$ & $-b_{1}[4], b_{3}[3]$ & $b_{2}[3], b_{1}[3]$ \\
\hline
\end{tabular}

Table 4.1: Symbol transmissions from different users in different time slots for three-user DF sharing protocol.

chronous communications, the extra communication overhead to maintain synchronization is not required. But we assume that the terminals transmit with exact same carrier frequency.

Table 4.1 contains a description of the data sent from each of the three users in one three-user sharing group, assuming one packet of data consists of four symbols from each user transmitted over six time slots. Here, the symbols transmitted from every user are separated by spreading code, but they could be separated by time or frequency. In the table, $b_{j}[i]$ denote $i$ 'th symbol of user $j$. As seen from the table, each user sends its own new data in every time slot. Thus, to start the transmission, user 1 sends $b_{1}[1]$ and $b_{1}[2]$ over first two time slots. Similarly users 2 and 3 send their own data in the first two time slots. During next two time slots (i.e., time slots 3 and 4), each user along with its new data sends other user's previous data using Alamouti space-time block code. For example, the space-time code formed by users 1 and 3 , denoted as $C_{13}$ is

$$
C_{13}=\left[\begin{array}{cc}
b_{2}[1] & b_{2}[2] \\
-b_{2}[2] & b_{2}[1]
\end{array}\right]
$$

In the above code matrix $C_{i j}$, the first row is transmitted by $i$-th user and the second row is transmitted by $j$-th user. Similarly users 2 and 3 will form a space-time block code to send user 1's data. The Alamouti space-time block code formed by users 2 and 3 can be represented as

$$
C_{23}=\left[\begin{array}{cc}
b_{1}[1] & b_{1}[2] \\
-b_{1}[2] & b_{1}[1]
\end{array}\right]
$$


and the code matrix transmitted by users 1 and 2 is

$$
C_{12}=\left[\begin{array}{cc}
b_{3}[1] & b_{3}[2] \\
-b_{3}[2] & b_{3}[1]
\end{array}\right]
$$

Effectively, the symbols transmitted by user 1 in the third time slots are $b_{2}[1], b_{3}[1]$ and $b_{1}[3]$. The continuous time signal transmitted by user 1 is thus

$$
b_{2}[1] s_{2}(t)+b_{3}[1] s_{3}(t)+b_{1}[3] s_{1}(t)
$$

where $s_{i}(t)$ represents spreading waveform of user $i$. Similar explanation can be given for symbols transmitted by other users. Again, during time slots 4 and 5, the users will form Alamputi space time block code using data received during previous two time slots.

We will show in Section 4.4 that by applying the receiver in Fig. 4.3 to the sharing schemes discussed in the previous sections result in a linear model similar to (4.13). Then we use the linear MMSE filter in (4.14), (4.15) for joint multiuser detection and space time decoding. Because of asynchronism, ML decoding of the orthogonal block codes is no longer linear, but we will see that linear reception, together with a non-linear complex conjugation receiver front-end, still provides full diversity.

Although distributed MIMO saves transmit power, it consumes significantly more processing power due to its complicated signal processing. We introduce a low-complexity receiver design to reduce the power consumption resulting due to complex decoding methods. In this section, we provide the design of a linear receiver which provides a near ML performance. Fig. 4.3 contains a block diagram of the proposed base-station receiver structure.

\subsection{Receiver Design}

To illustrate every block of the base-station receiver structure, we consider the received signal resulting from two-user decode-and-forward cooperation scheme as input to our receiver. But the same receiver structure can also be applied to three-or-more-user cooperation and amplify-and-forward cooperation schemes explained in the following sections. 


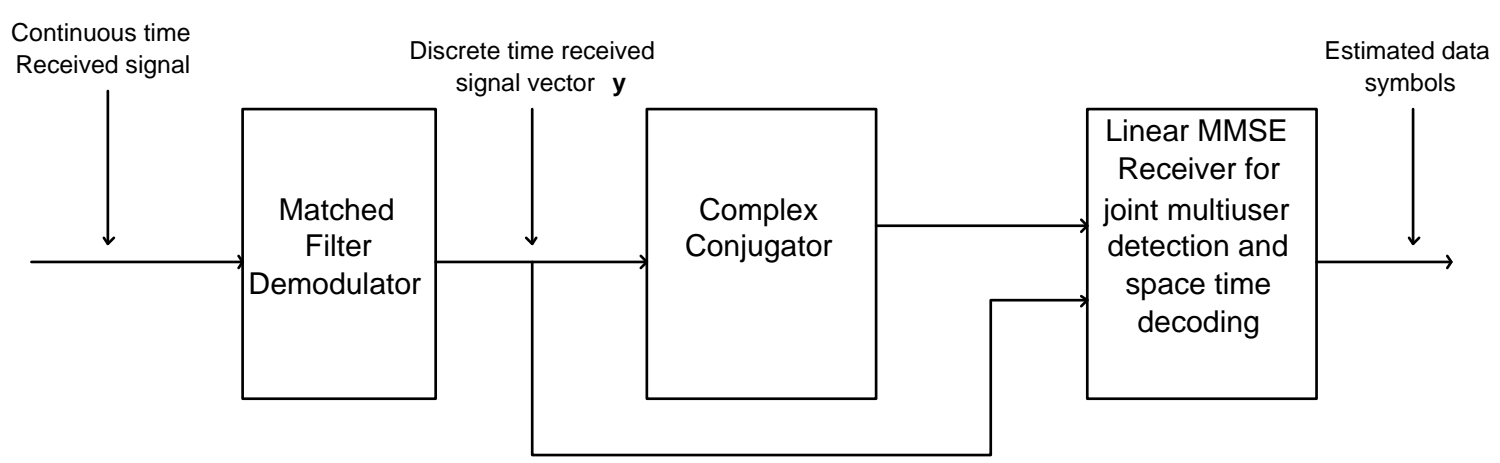

Figure 4.3: Block Diagram of the Receiver Structure

The received signal at the base station over the three time slots, for two-user sharing scheme is then

$$
\begin{aligned}
r(t)= & \alpha_{1} b_{1}[1] s_{1}\left(t-\tau_{1}\right)+\alpha_{2} b_{2}[1] s_{2}\left(t-\tau_{2}\right)+\alpha_{1} b_{1}[2] s_{1}\left(t-T_{s}-\tau_{1}\right)+ \\
& \alpha_{1} b_{2}[1] s_{2}\left(t-T_{s}-\tau_{1}\right)+\alpha_{2} b_{2}[2] s_{2}\left(t-T_{s}-\tau_{2}\right)+\alpha_{2} b_{1}[1] s_{1}\left(t-T_{s}-\tau_{2}\right)+ \\
& +\alpha_{1} b_{2}[2] s_{2}\left(t-2 T_{s}-\tau_{1}\right)+\alpha_{2} b_{1}[2] s_{1}\left(t-2 T_{s}-\tau_{2}\right)+n(t)
\end{aligned}
$$

where $\alpha_{1}\left(\alpha_{2}\right)$ is the complex Gaussian channel gain between user 1 (user 2) and the base station, $\tau_{1}$ and $\tau_{2}$ are the corresponding delays, and $n(t)$ is a white Gaussian noise process. Also, $s_{k}(t)=\sum_{j=0}^{N-1} c_{k}[j] \psi\left(t-j T_{c}\right)$, where $c_{k}[j]$ is the $j$-th element of user $k$ 's spreading code and $\psi(t)$ is a unit-energy rectangular chip pulse waveform. $T_{s}$ is the symbol duration and $T_{c}=T_{s} / N$ is the chip period. At the receiver, as shown in Fig. 4.3, we first perform matched filter detection with respect to pulse shape $\psi(t)$. Thus the matched filtered output during $i$-th time slot and $q^{\text {th }}$ chip interval is given by [45]

$$
y[i, q]=\int_{i T_{s}+q T_{c}}^{i T_{s}+(q+1) T_{c}} r(t) \psi\left(t-i T_{s}-q T_{c}\right) d t+n[i, q]
$$

where $n[i, q]$ is the complex Gaussian noise. Note that matched filtering with respect to $\psi(t)$ does not necessarily generate a sufficient statistic in asynchronism (this would require match filtering with respect to $\psi(t)$ convolved with the channel). However, match filtering with respect to $\psi(t)$ does not require a priori knowledge of the delays and path gains, which simplifies channel estimation.

When we deal with asynchronism $\left(\tau_{1}, \tau_{2} \in\left[0, T_{s}\right]\right)$, we match filter the continuous time received signal for one extra time slot. The discrete time received signal vector can thus be 
obtained by stacking all the matched filter outputs from (4.8), producing

$$
\boldsymbol{y}=[y[0,0] \ldots y[0, N-1] \ldots y[3,0] \ldots y[3, N-1]]^{T}
$$

which can be written as the linear model

$$
\boldsymbol{y}=\boldsymbol{H} \boldsymbol{s}+\boldsymbol{n}
$$

where $\boldsymbol{H}$, a function of the spreading codes and the channel gains, is the effective channel matrix, $s$ is the BPSK symbol vector given by

$$
\boldsymbol{s}=\left[\begin{array}{llll}
b_{1}[1] & b_{1}[2] & b_{2}[1] & b_{2}[2]
\end{array}\right]^{T},
$$

and $\boldsymbol{n} \sim \mathcal{N}_{c}\left(0, \sigma^{2} \boldsymbol{I}\right)$. A detailed description of discrete time linear model given in (4.10) can be found in Appendix B. We then form a new discrete time received signal vector $\tilde{\boldsymbol{y}}$ by stacking complex conjugate version of $\boldsymbol{y}$ with the conventional received signal vector $\boldsymbol{y}$, i.e.,

$$
\begin{aligned}
\tilde{\boldsymbol{y}}=\left[\begin{array}{c}
\boldsymbol{y} \\
\boldsymbol{y}^{*}
\end{array}\right] & =\underbrace{\left[\begin{array}{c}
\boldsymbol{H} \\
\boldsymbol{H}^{*}
\end{array}\right]}_{\tilde{\boldsymbol{H}}} s+\underbrace{\left[\begin{array}{c}
\boldsymbol{n} \\
\boldsymbol{n}^{*}
\end{array}\right]}_{\tilde{\boldsymbol{n}}} . \\
& =\tilde{\boldsymbol{H}} \boldsymbol{s}+\tilde{\boldsymbol{n}} .
\end{aligned}
$$

The complex conjugation step is critical to obtaining full diversity with linear reception. This can be explained as follows. Let's consider Alamouti's code which is a special case of orthogonal space-time codes with two transmit antennas. As seen from (3.15), ML decoding of orthogonal ST codes is accomplished by complex conjugation of some received signal components followed by a linear transformation. Because linear ML detection of orthogonal space-time block codes requires complex conjugates of the received signal, it is reasonable to assume complex conjugates will be helpful for linear detection in asynchronous communication environments. Complex conjugation is therefore necessary for the same reason it is needed before the linear transformation that decodes orthogonal space-time block codes. We then perform joint multiuser detection and space time decoding with a linear MMSE filter operating on $\tilde{\boldsymbol{y}}$. Our decision statistic is formed as

$$
\boldsymbol{d}=\left[\left[\tilde{\boldsymbol{H}} \tilde{\boldsymbol{H}}^{\mathrm{H}}+\sigma^{2} \boldsymbol{I}\right]^{-1} \tilde{\boldsymbol{H}}\right]^{\mathrm{H}} \tilde{\boldsymbol{y}}
$$


with bit decisions provided by

$$
\hat{\boldsymbol{s}}=\operatorname{sign}[\Re\{\boldsymbol{d}\}] .
$$

The expression for MMSE linear filter is derived in the Appendix C. We observe that, to form a detector, an explicit knowledge of individual delays is not required at the base station receiver. The receiver performs joint linear multiple-access interference suppression and space-time decoding. It has the advantages of low complexity and is easy to make adaptive.

\subsection{Amplify and Forward Cooperation}

The AF protocol presented here is comparatively simple. Due to reciprocity of the channel, we assume that the channel between user $i$ and user $j$ is the same as channel between user $j$ and user $i$. We further assume that the inter-user channel SNR is the same as the SNR of the channel between users and the base station, i.e., we assume perfect power control. The base station is assumed to have knowledge of the inter-user channel fading coefficients. The protocol works as follows. Each user receives other user's data over a noisy channel. The received data is a corrupted version of the transmitted data. Each user then forwards this noisy received signal from the previous time slot to base station along with its own data at the current time slot. The authors in [2] provide an expression for the gain with which the relay terminals should amplify the received signal before forwarding it. The value of this gain is dependent upon the fading coefficient between the source and relay. This requires channel estimation at the relay node. We use unity gain for simulations. Table 4.2 contains the description of data symbols sent from two users in one two-user sharing

\begin{tabular}{|l|l|l|}
\hline Time Slot & User1 & User 2 \\
\hline \hline 0 & $b_{1}[1]$ & $b_{2}[1]$ \\
\hline 1 & $b_{1}[2]+\alpha_{12}\left(b_{2}[1]+n_{1}\right)$ & $b_{2}[2]+\alpha_{12}\left(b_{1}[1]+n 2\right)$ \\
\hline 2 & $\alpha_{12}\left(b_{2}[2]+n_{3}\right)$ & $\alpha_{12}\left(b_{1}[2]+n_{4}\right)$ \\
\hline
\end{tabular}

Table 4.2: Symbol transmissions from different users in different time slots for two-user AF sharing protocol. 
group where $\alpha_{12}$ is the complex Gaussian channel gain of the inter-user channel. Similar description can be given for three-user AF scheme.

\subsection{Simulation Results}

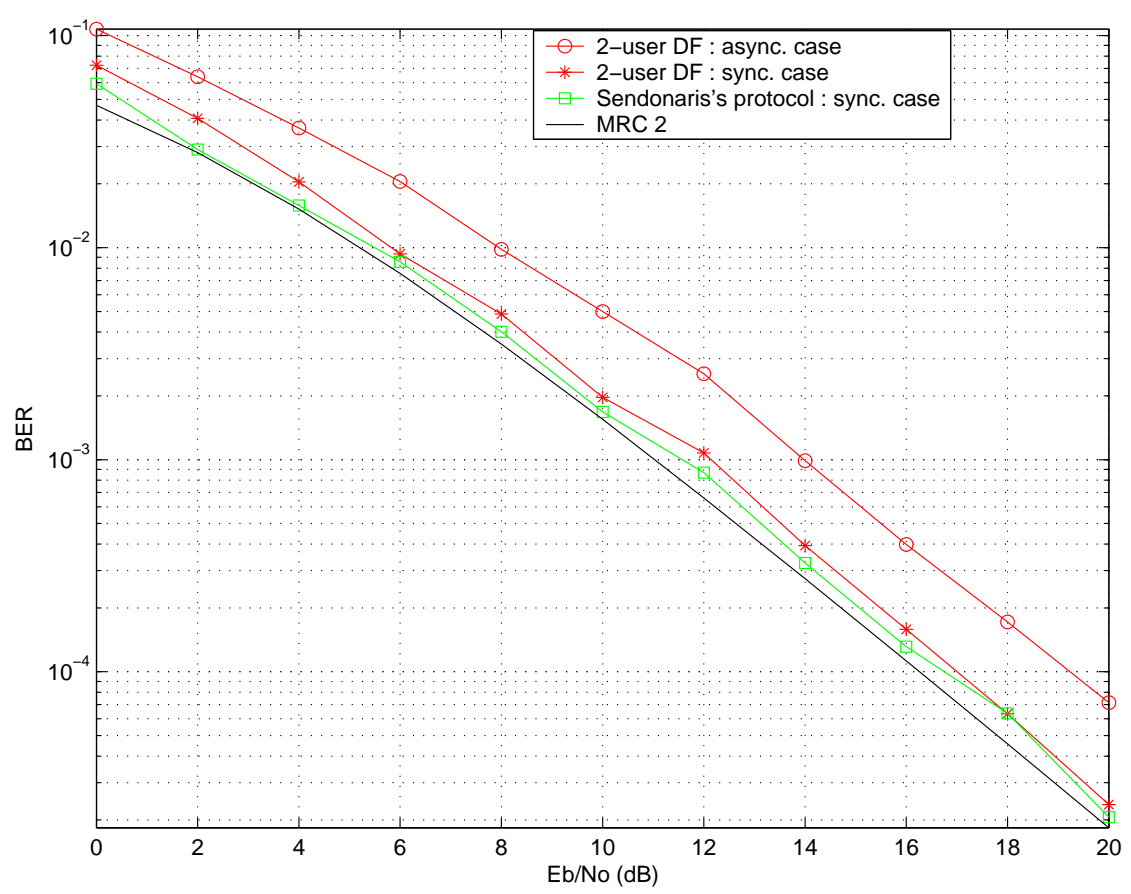

Figure 4.4: BER and diversity performance of two-user DF sharing in synchronous and asynchronous uplink environments. The BER curve for the Sendonaris protocol is plotted for comparison, as is the performance of maximum-ratio combining with 2 antennas (MRC $2)$.

We use random spreading codes of length 8 . The normalized total transmit power of each user during each time slot is 1 . We assume perfect channel knowledge to be available at the base station. Fig. 4.4 illustrates the BER performance of the two-user DF sharing scheme in synchronous and asynchronous uplink environments. The Sendonaris protocol performance, along with maximum-ratio combining (MRC) with two antennas, are provided for comparison purposes. There are variations of Sendonaris' protocol, depending upon the duration of cooperation. For Sendonaris's protocol, the rate of the system decreases but the diversity gain increases as the cooperation period increases. Thus there exists a rate-diversity gain trade-off for his schemes. For comparison purposes, we consider a version where users in 


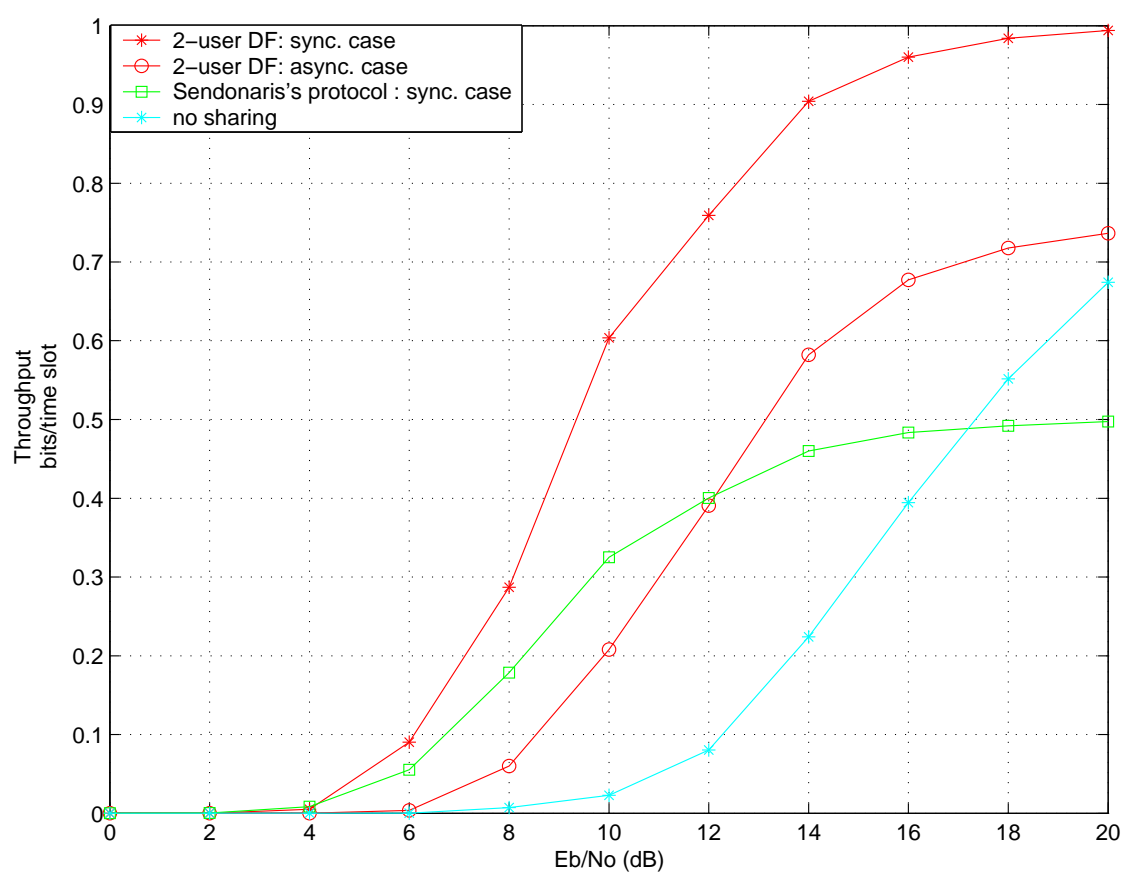

Figure 4.5: Throughput performance of two-user DF sharing in synchronous and asynchronous uplink environments. The throughput curve for the Sendonaris protocol and no sharing is plotted for comparison.

the system fully cooperate, giving full diversity gain (diversity order $=2$ ), but operate at rate $=1 / 2$. All curves indicate, via a comparison with the slope of maximum-ratio combining, that full diversity is achieved. It can be seen that, when delays are uniformly distributed in the range $\left[0, T_{s}\right]$, two-user sharing scheme suffers from approximately $1 \mathrm{~dB}$ SNR loss as compared to synchronous case but it still provides full diversity gain with a linear receiver.

Fig. 4.5 illustrates the normalized throughput performance of the two-user DF protocol, along with Sendonaris and no-sharing. Throughput is specified by the number of successfully received information bits per transmission. Normalized throughput, $T_{h}$, is calculated by using equation (3.11) where we use $M=256$ as the packet size. The achievable throughput mainly depends on the BER and the rate of the system. For very low values of BER (and hence, at high SNRs), throughput is large and as can be observed from the equation (3.11), is only limited by the rate $R$. As discussed before in this chapter, the proposed sharing schemes operate in full-duplex mode where each cooperating user transmits a new symbol every time slot. Hence the rate of the system is limited to 1 , in synchronous environments ${ }^{3}$.

\footnotetext{
${ }^{3}$ It is possible to increase the rate of the system $(R>1)$ using BLAST techniques, where M symbols
} 


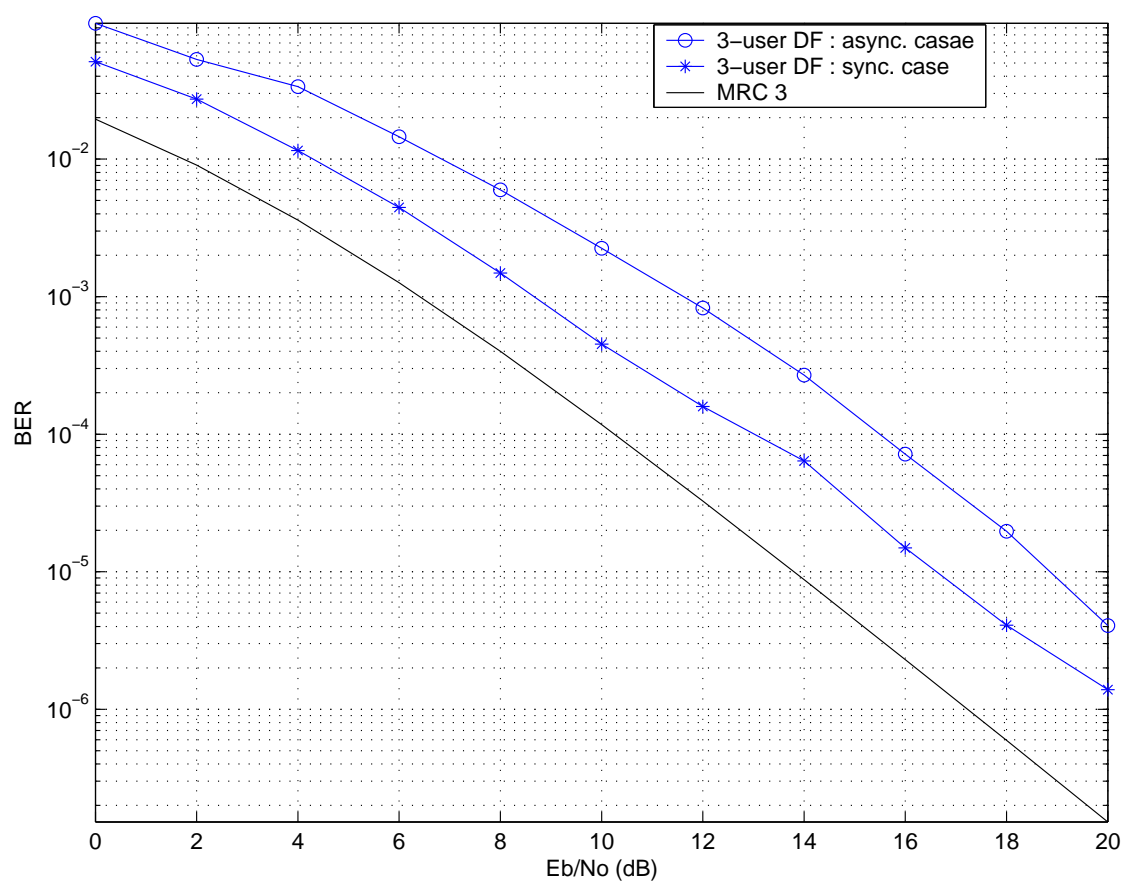

Figure 4.6: BER performance of three-user DF sharing in synchronous and asynchronous uplink environments. The BER performance of maximum-ratio combining with three antennas (MRC 3) is provided for comparison.

If we assume asynchronous channels, then due to zero padding between the data packets, the rate will be less than 1 . Therefore, the throughput for the sharing schemes discussed in this chapter is limited to 1 . The proposed protocols can operate at full rate in a synchronous uplink $(R=1)$ giving full diversity simultaneously and hence does not exhibit a trade-off between rate and the diversity gain. In asynchronism, the two-user protocol operates at $R=3 / 4$ while the three-user protocol operates at $R=6 / 7$ due to zero padding between packets. No-sharing always operates at $R=1$ and the full-diversity version of the Sendonaris protocol operates at $R=1 / 2$. Notice that the proposed schemes outperform Sendonaris and no-sharing for all SNRs of interest. We also see that all sharing curves indicate higher throughput performance than is obtained for no-sharing, at least for low and moderate SNR values. The maximum achievable throughput using two user sharing scheme in asynchronous case is 0.75 (considering one block of data to be consisting of transmission over three time slots), since we use zero padding after each block of data. It is possible to increase this rate without using zero padding technique, but this may lead to SNR loss. Also it is seen that, at could be transmitted every time slot, but is not the topic of interest here. 


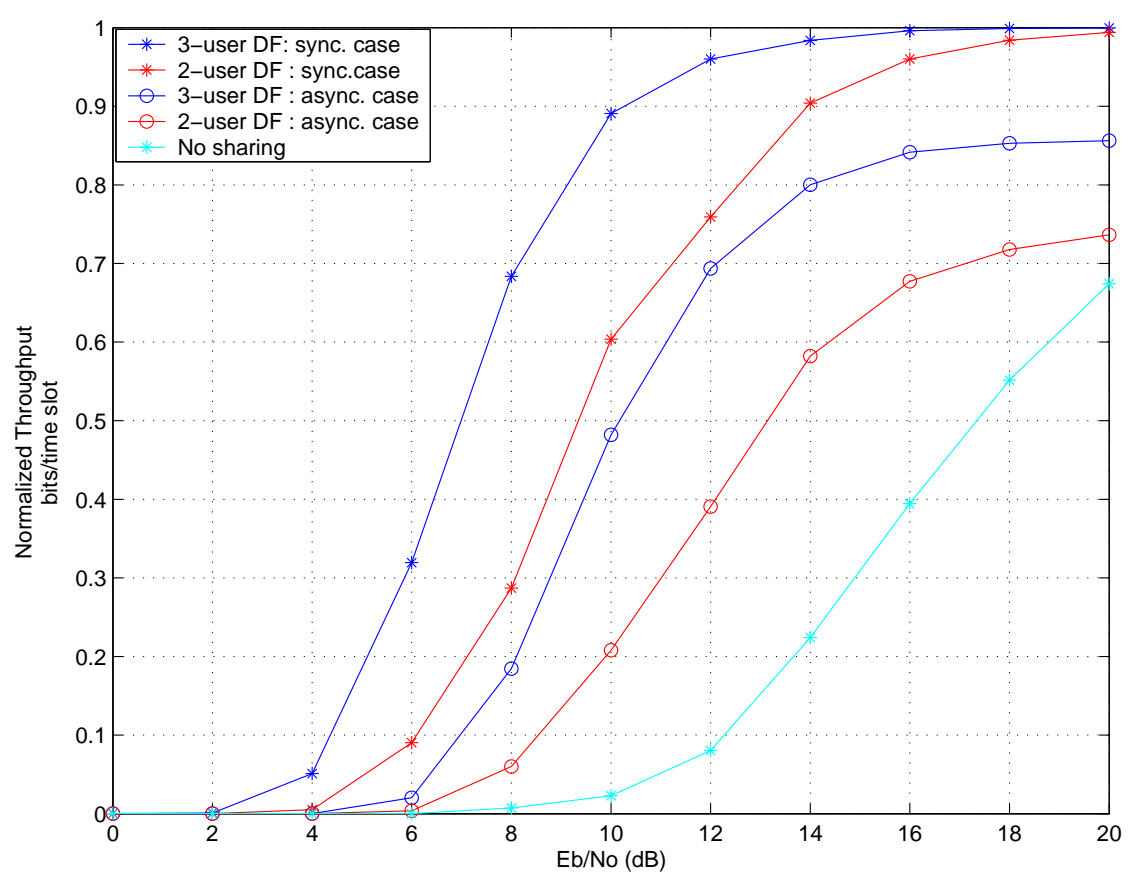

Figure 4.7: Throughput performance of two-user and three-user DF sharing in synchronous and asynchronous uplink environments. Also included is the throughput performance without sharing.

high SNRs, the two user sharing scheme operating in asynchronous communication systems has better throughput performance than Sendonaris's protocol operating in synchronous communications.

Figures 4.6 and 4.7 provide similar simulation results for three-user sharing using a distributed version of the Alamouti space-time block code. Here, a comparison is made with our two-user DF protocol since the Sendonaris protocol is not generalizable to more than 2 users. It shows that the three-user sharing protocol performs better than the two-user sharing protocol in both the synchronous and asynchronous situations. BER and throughput curves for AF sharing appear in Figures 4.8 and 4.9, respectively. As the number of cooperating users in the system increases, the AF protocol shows degradation in performance, indicating that our receiver design is not effective for more than two user AF sharing. Also, results show that, when perfect bit estimates are available at the user side and all the communication links between users and a base station have the same SNR, DF protocol works better than the corresponding AF protocol. Clearly, under the present system constraints, DF uniformly outperforms AF. Both DF and AF protocols offer advantages over no sharing in the system. 


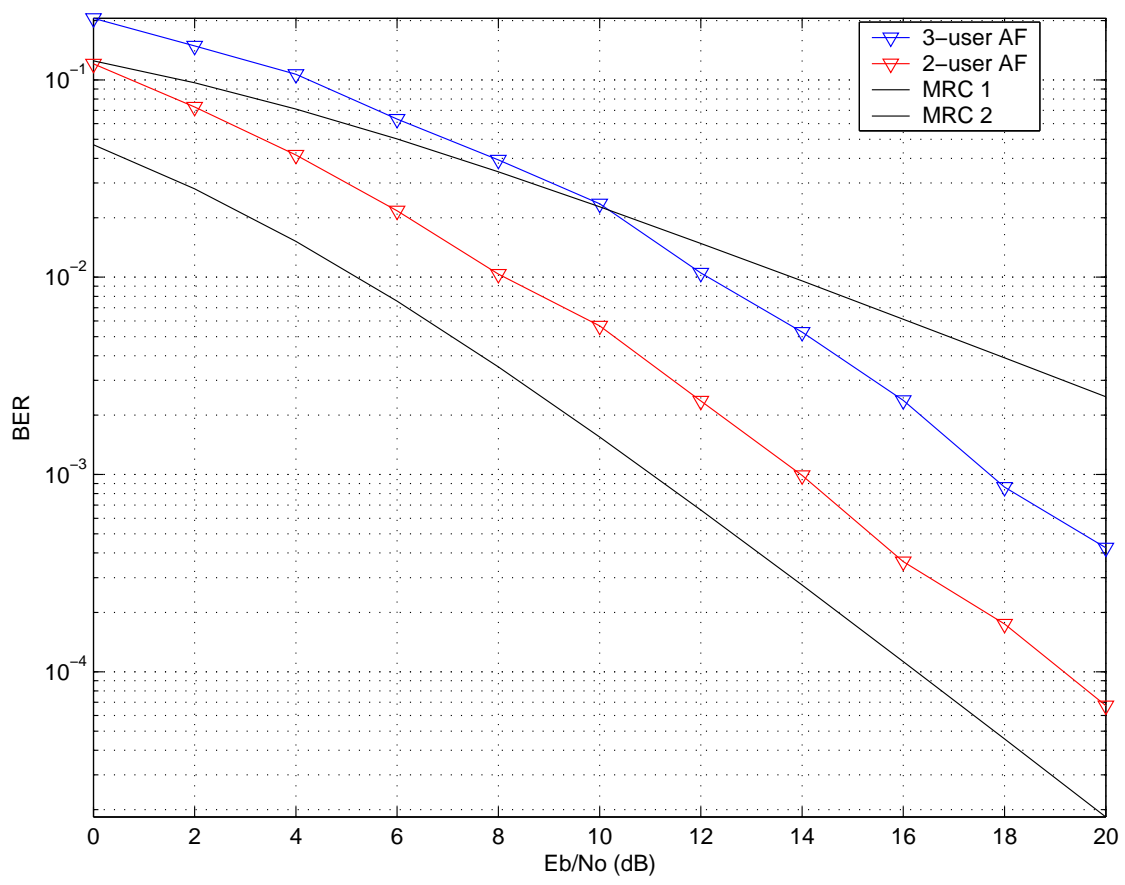

Figure 4.8: BER performance of two-user and three-user AF sharing in a synchronous uplink. The performance of maximum-ratio combining with 1 and 2 antennas is also included for comparison.

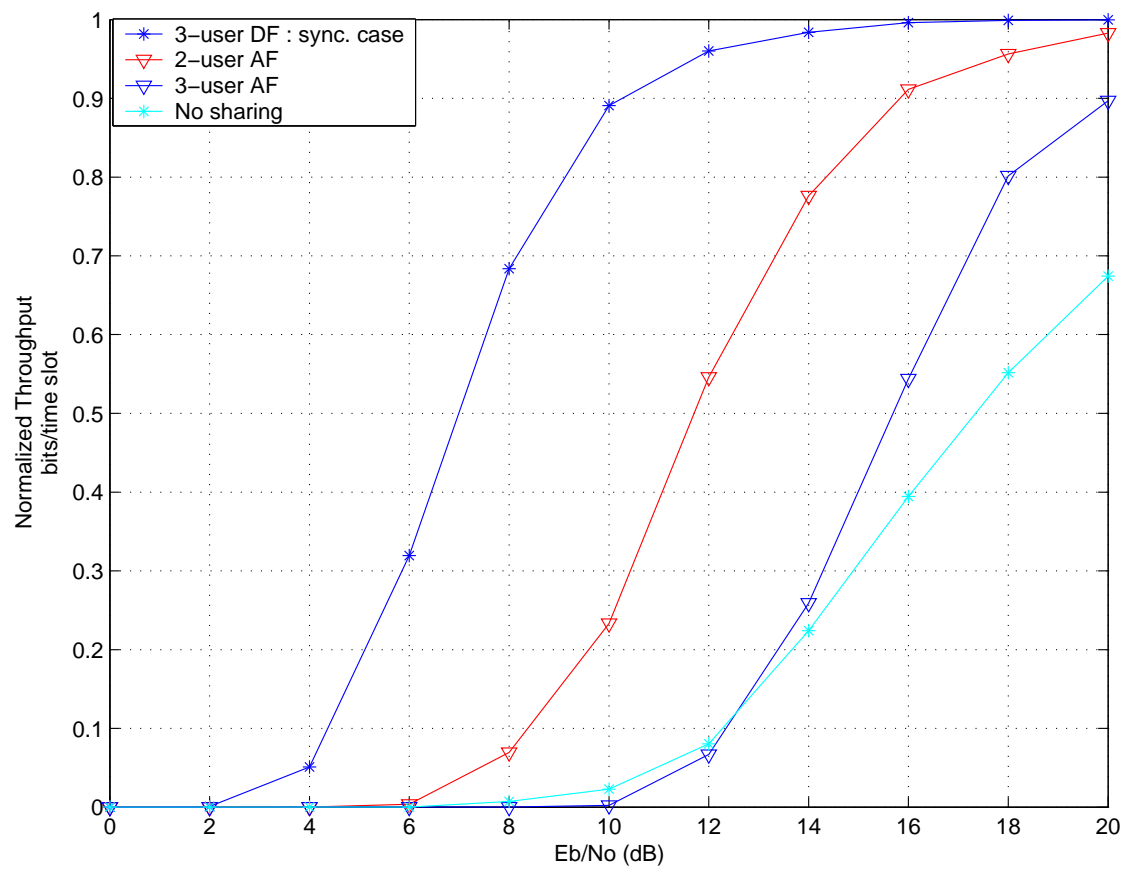

Figure 4.9: Throughput performance of two-user and three-user AF sharing in synchronous uplink. Also included is the throughput performance achieved without sharing. 
We note in passing that AF protocols can also be space-time coded, which may lead to different conclusions.

\subsection{Conclusion}

In this chapter, we developed new multiuser sharing protocols for diversity and throughput enhancement in an asynchronous cellular uplink. The DF schemes provide near ML performance, including full diversity and significant throughput improvements over no-sharing and existing schemes, e.g., Sendonaris et al, under low-complexity linear joint multiuser detection and space-time decoding. The proposed schemes can easily be extended to cooperating user groups of any size using orthogonal or non-orthogonal space-time block codes. An AF protocol was also simulated for comparison purposes and was found to be uniformly inferior to the DF protocols under the system constraints provided. But in the situations where perfect bit estimates are not available at the user side and when there exists some power control technique, it may be possible for AF protocol to work better than the DF protocol. The issue of imperfect inter-user channels will be addressed in the next chapter. 


\section{Chapter 5}

\section{Multi-User DF Sharing Schemes with Noisy Inter-User Channels}

Cooperative relaying, whereby source nodes multicast a message to a number of helping (cooperating) nodes which in turn resend a processed version of the received signal to the intended destination node, is the most promising architectural upgrade from the conventional multi-antenna systems. It offers advantages such as radio range extension in cellular networks and acts as a means to reduce infrastructure deployment costs and enhance capacity of the network. In the last chapter, new cooperative diversity strategies for the cellular uplink were presented, along with an appropriate receiver design and simulation results that demonstrated significant performance gains over existing multiuser cooperative strategies. In the previous chapter, we assumed inter-user channels with high signal-to-noise ratio whereby cooperating users were able to decode other user's data successfully. We need to check the performance improvements due to cooperation between users when more realistic constraints are posed. In this chapter, we consider practical noisy inter-user channels. Cooperative diversity with noisy inter-user channels has been studied in [7]. Authors in [7] consider the case when the cooperating partners are equipped with more than one antenna. They consider a situation where upon unsuccessful reception, partners notify the source about it and the source then transmits the rest of the data instead of relaying on cooperation from its partner. In [8], authors perform asymptotic analysis of cooperative space-time coded system

\footnotetext{
${ }^{1}$ Part of the material presented in this chapter has been accepted in[46] and it also appears in [47]
} 
in order to determine the achievable diversity gain through cooperative space time coding for various inter-user channel qualities. But the prior work is restricted to only two-user sharing and synchronous communication environments and does not specify the receiver structure. In this chapter, we evaluate the performance of multi-user DF sharing schemes in the presence of imperfect inter-user channels, considering asynchronous communication scenario. We provide an adaptive receiver structure and additional bit-error-probability and throughput simulations for more practical, noisy inter-user channels. We will see that the two-user sharing scheme fully exploits available diversity with inter-user demodulation errors as high as $0.1 \%$ using linear joint space-time decoding and multiuser detection.

\subsection{System Model}

Similar to the last chapter, we consider a BPSK modulated CDMA cellular uplink consisting of cooperating users that are grouped by higher layer protocol. All mobile units including the base station are equipped with a single antenna and asynchronous communication is assumed. We assume a system with cyclic redundancy check (CRC) coding for error detection at the mobile units. Cooperating users in the system receive data from the source. They perform CRC check to determine if the received frame is in error. We model our system such that cooperating users do not forward frames that contain errors. Fixed decode and forward protocols (or demodulate and forward protocols, where users forward the decoded data without CRC check) are not enough to achieve diversity gains because incorrect decisions by cooperating users propagate to the destination. A performance improvement is obtained if helping nodes never forward frames in error.

We use zero padding between successive frames of data to avoid inter-block interference. The spreading codes provide processing gain $N$ and they are not assumed orthogonal unlike previous related works [5], [6]. The AWGN noise power is $\sigma^{2}$ and we assume perfect power control so that the Rayleigh flat-fading channels between users and the base station are assumed statistically identical. But we examine the effect of inter-user channel quality variation on the BER and hence diversity performance. Channel state information is obtained at the base station through adaptive channel estimation at the base station receiver. Also, 
the channel is assumed to be slowly varying. Linear joint MMSE multiuser detection and space-time decoding is then performed at the receiver as discussed in Chapter 4. Simulation results indicate that although an expected SNR loss is incurred, there is no loss in diversity gain using the adaptive structure or due to inter-user errors, as long as the inter-user BER is less than 0.04 .

\subsection{Two-User Decode and Forward Cooperation}

Here we consider a $K$-user system where the users have been grouped into pairs for uplink transmission. In each time slot, each user transmits its own data on its spreading code. It performs a CRC check on its partners previous data (data received in the previous time slot). If the frame has been received without any error, then it also transmits its partner's previous data on that user's spreading code, otherwise it just discards the partner's data. Thus, symbols transmitted from two users during the $n$-th time slot are

$$
\begin{array}{ll}
\text { User } 1: & b_{1}[n] s_{1}+\beta_{1} b_{2}[n-1] s_{2} \\
\text { User 2: } & b_{2}[n] s_{2}+\beta_{2} b_{1}[n-1] s_{1}
\end{array}
$$

where, $b_{j}[i]$ is the symbol transmitted from user $j$ at time slot $i$ and $s_{k}$ is the signature waveform of user $k$. $\beta_{1}$ and $\beta_{2}$ are the Bernoulli random variables having values 0 or 1 and they represent the user's decision (made via CRC check) on forwarding partner's data.

\subsection{Three-or-More User Decode and Forward Cooper- ation}

Here, we develop a specific protocol for three or more users that makes use of space-time block codes $[24,25]$. As an example, we consider a $\mathrm{K}$ user system whose users have been assigned to groups of three. Similar to the two-user protocol, each user sends its own new data in every time slot. Simultaneously, upon successful decoding, each user transmits the other users' previous data using a distributed space-time block code. In particular, if user 1 is able to estimate users 2 and 3's data, it will form a space time code with user 2 to send user 3's previous data and user 1 will also form a space time code with user 3 to send user 


\begin{tabular}{|l|l|l|l|}
\hline Time Slot & User1 & User 2 & User 3 \\
\hline \hline 0 & $b_{1}[1]$ & $b_{2}[1]$ & $b_{3}[1]$ \\
\hline 1 & $b_{1}[2]$ & $b_{2}[2]$ & $b_{3}[2]$ \\
\hline 2 & $\beta_{1} b_{2}[1], \beta_{2} b_{3}[1], b_{1}[3]$ & $\beta_{3} b_{1}[1], \beta_{4} b_{3}[2], b_{2}[3]$ & $\beta_{5} b_{1}[2], \beta_{6} b_{2}[2], b_{3}[3]$ \\
\hline 3 & $-\beta_{7} b_{2}[2],-\beta_{8} b_{3}[2], b_{1}[4]$ & $-\beta_{9} b_{1}[2], \beta_{10} b_{3}[1], b_{2}[4]$ & $\beta_{11} b_{2}[1], \beta_{12} b_{1}[1], b_{3}[4]$ \\
\hline 4 & $\beta_{13} b_{2}[3], \beta_{14} b_{3}[3]$ & $\beta_{15} b_{1}[3], \beta_{16} b_{3}[4]$ & $\beta_{17} b_{1}[4], \beta_{18} b_{2}[4]$ \\
\hline 5 & $-\beta_{19} b_{2}[4],-\beta_{20} b_{3}[4]$ & $-\beta_{21} b_{1}[4], \beta_{22} b_{3}[3]$ & $\beta_{23} b_{2}[3], \beta_{24} b_{1}[3]$ \\
\hline
\end{tabular}

Table 5.1: Symbol transmissions from different users in different time slots for three-user DF sharing protocol assuming noisy inter-user channel.

2's previous data. If user 1 is not able to decode either user 2's or user 3's data successfully, it will not transmit that user's data during the appropriate time slot of the space-time code. For three-user sharing, we make use of popular Alamouti space time code for the same reasons as explained in the last chapter.

Table 5.1 contains a description of the data sent from each of the three users in one three-user sharing group, assuming one frame of data consists of four symbols from each user transmitted over six time slots.

In the above table, $\beta_{n}$ 's, $n \in \mathbb{N}$ are the Bernoulli random variables having values 0 or 1 and they represent the user's decision (made via CRC check) on forwarding partner's data, similar to two-user sharing case.

\subsection{Receiver Design}

The receiver structure proposed in Chapter 4 consists of a signal-independent non-linear component (a complex conjugator) and a linear MMSE joint space-time decoder and multiuser detector. Results presented in Chapter 4 indicate that full diversity can be achieved for asynchronous transmission, assuming perfect knowledge of the channel matrix at the base station. In this chapter, we present adaptive receiver structure. Since cooperating users make random estimation errors in the data frames they forward and the base station can not have the knowledge of the errors or the inter-user channels, adaptive receiver structures play a crucial role in situations where inter-user channel is not perfect. We consider adaptive receivers comprising channel estimation method. The estimation of the channel 
involves estimation of channel delays and channel attenuation factors. These techniques can be broadly divided into two categories: (1) Blind channel estimation, (2) Training based channel estimation approach. Blind channel estimation techniques involve estimating channels using only the received signal. They have the advantages of being bandwidth efficient and robust to fast fading. We note that not all blind channel estimation techniques are robust to fast fading, e.g., blind techniques based on high-order statistical methods (due to their long convergence time). But there exist blind techniques for channel estimation that require a very few data samples as compared to training based methods for the estimator to converge (e.g. channel estimation based on deterministic approach, instead of statistical approach), making them attractive in fast fading channel conditions. Because the effective channel matrix seen by the base station includes the effects of the inter-user channels and products of random variables, conventional blind channel-estimation techniques may not apply. Here, we present an adaptive structure based on existing channel estimation schemes using periodically-inserted pilot symbols.

Training based schemes are composed of two phases: (i) Training phase and (ii) Data Transmission phase. The data sent during the training phase is known at the receiver while it is unknown during the data transmission phase. Suppose $T_{\text {train }}$ denotes the time required to train the system and $T_{\text {data }}$ represents the time devoted to actual data transmission. It is clear that increasing $T_{\text {train }}$ improves the quality of the channel estimate. But if more time is spent in the training process, then little time is set aside for data transmission. Allowing small training period degrades the quality of the channel estimates but that can be counterbalanced by increasing the training symbol power. The authors in [48] found the optimal solution for training interval in terms of number of transmit and receive antennas, SNR at the receivers and the coherence time of the channel where they assume training and data powers to be variable.

In many training based receivers, the received signal and the known training sequence is used to detect actual data symbols without explicitly forming a channel estimate. In other types of receivers which are training based, the estimated channel is often considered as if it were a true channel and is then used to form a detector. From equation (4.10), we know that the discrete time received signal model at the base station for $K$-user decode and forward 
schemes can be written in the form

$$
\boldsymbol{y}=\boldsymbol{H} \boldsymbol{s}+\boldsymbol{n}
$$

where $\boldsymbol{H}$, a function of the spreading codes, the channel gains and the random data forwarding decisions of the cooperating users, is the effective channel matrix, $s$ is the BPSK symbol vector and $\boldsymbol{n} \sim \mathcal{N}_{c}\left(0, \sigma^{2} \boldsymbol{I}\right)$. If we consider the first training based approach where channel is not estimated explicitly, then using Wiener-Hopf equation, the linear MMSE detector is given by [49]

$$
\boldsymbol{W}=\boldsymbol{R}^{-1} \boldsymbol{P}
$$

where $\boldsymbol{R} \triangleq E\left\{\boldsymbol{y} \boldsymbol{y}^{H}\right\}$ is the auto-correlation matrix of the received signal and $\boldsymbol{P} \triangleq E\left\{\boldsymbol{y} \boldsymbol{s}^{H}\right\}$ is the cross-correlation between desired response and the received signal. The receiver replaces the expectations in the above formulae with time averaging operation.

The other training based approach explicitly estimates the channel and then uses channel estimates to form a detector. Fig. 5.1 compares two training based methods for detection of transmitted data. It can be observed that the training based approach which estimates the channel explicitly has better BER performance in terms of SNR gain as compared to other method in which training sequence is used for joint channel estimation, multiple access interference suppression and space time decoding, although diversity performance remains the same.

For the reasons explained, we deal with the training based channel estimation problem where data and training powers are the same. We assume that a known sequence of training symbols is available. Before start of actual transmission of information, the source sends training sequence over the fading channel. Assuming coherence time $\left(T_{c o h}\right)$ of the channel to be greater than the training period and the actual transmission period, the base station receiver estimates the channel because it has the knowledge of training symbols. Since the channel model is a block fading channel model, we assume that the channel estimation (via training) and data transmission is done within the interval $T_{e s t}\left(T_{e s t}<T_{c o h}\right)$, after which new training allows us to estimate the channel for next block of data ( $T_{\text {est }}$ symbols) and so on. The estimate of the effective channel matrix $\boldsymbol{H}$ using least square method is then given by $[50]$ 


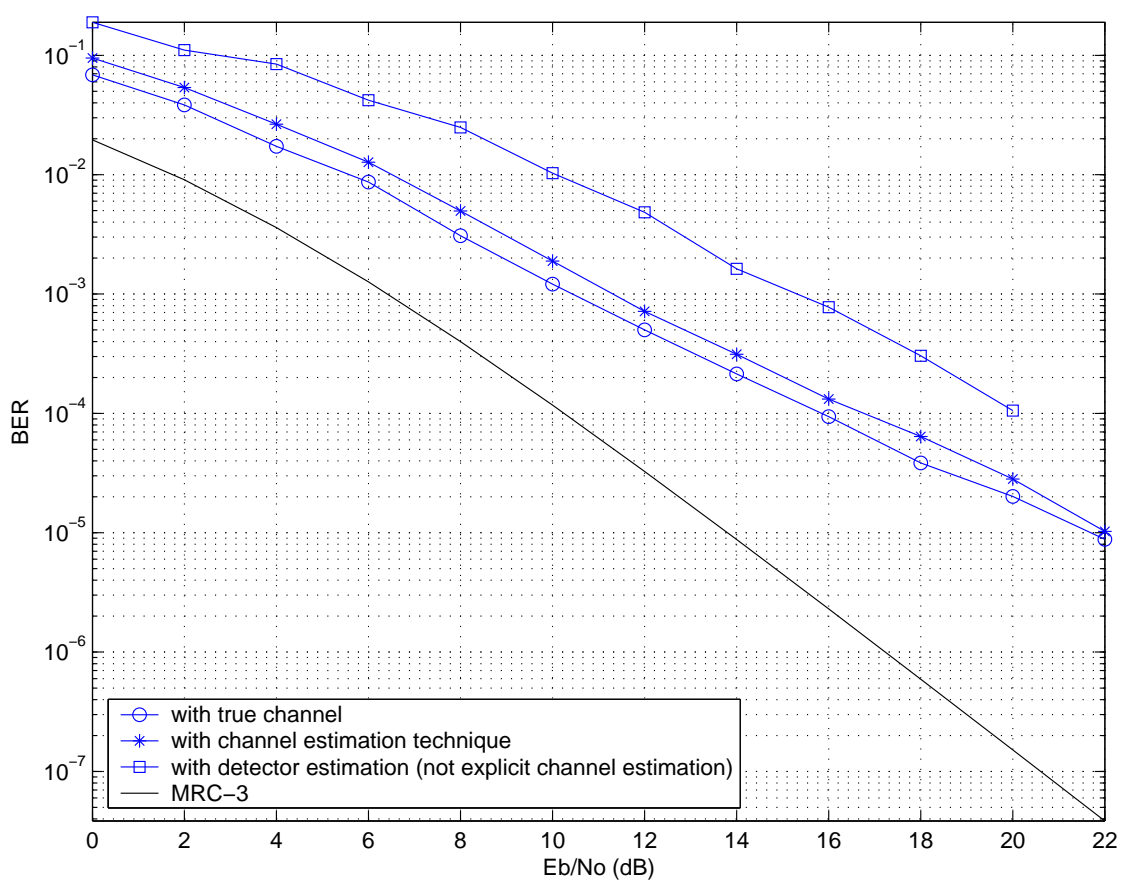

Figure 5.1: Performance comparison of two training based methods. Three-user sharing protocol operating in synchronous environments with $4 \%$ demodulation error is considered.

$$
\hat{\boldsymbol{H}}=\hat{\boldsymbol{R}}_{y s} \hat{\boldsymbol{R}}_{s s}^{-1}
$$

where,

$$
\hat{\boldsymbol{R}}_{y s}=\frac{1}{N} \sum_{N} \boldsymbol{y} \boldsymbol{s}^{H}
$$

is the sample cross-correlation matrix between the transmitted signal vector and the received signal vector.

and

$$
\hat{\boldsymbol{R}}_{s s}=\frac{1}{N} \sum_{N} s s^{H}
$$

is the sample auto-correlation matrix of transmitted signal vector. The proof of the channel estimation problem appears in the Appendix D.

The estimated channel is then used to form a detector given in (4.14). The adaptive receiver thus involves training the system first and hence there is a loss in rate (because 
training symbols do not carry any information). This type of receiver is useful when the channel is slowly varying.

\subsection{Simulation Results}

We present simulation results for our two and three user sharing protocols operating in synchronous as well as asynchronous uplink with inter-user demodulation errors.

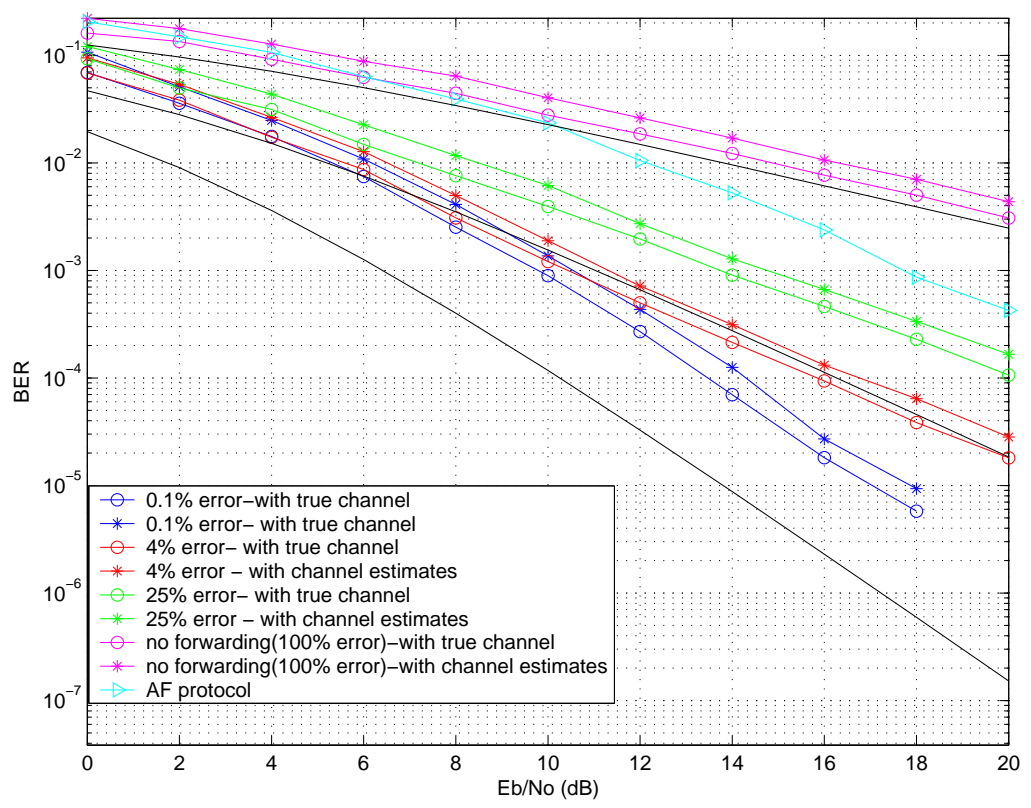

Figure 5.2: BER performance of three-user DF sharing in a synchronous uplink. The performance of maximum-ratio combining with 2 and 3 antennas is also included for comparison.

The base-station receiver operates in batch adaptive mode. It uses a set of 150 frames of data to estimate the effective channel, forms a detector using the channel estimates and then applies the detector to data frames. Increasing the number of frames for training to obtain a channel estimates reduces the SNR loss relative to the case of perfectly known channel. The number of frames used to estimate the channel does not affect the diversity performance of the system. The results are included for a perfectly known channel and for an estimated channel. For all simulations, we assume that the channels between each user and the base station have the same average SNR. We use random spreading codes of length 8. The normalized total transmit power of each user during each time slot is 1 . 


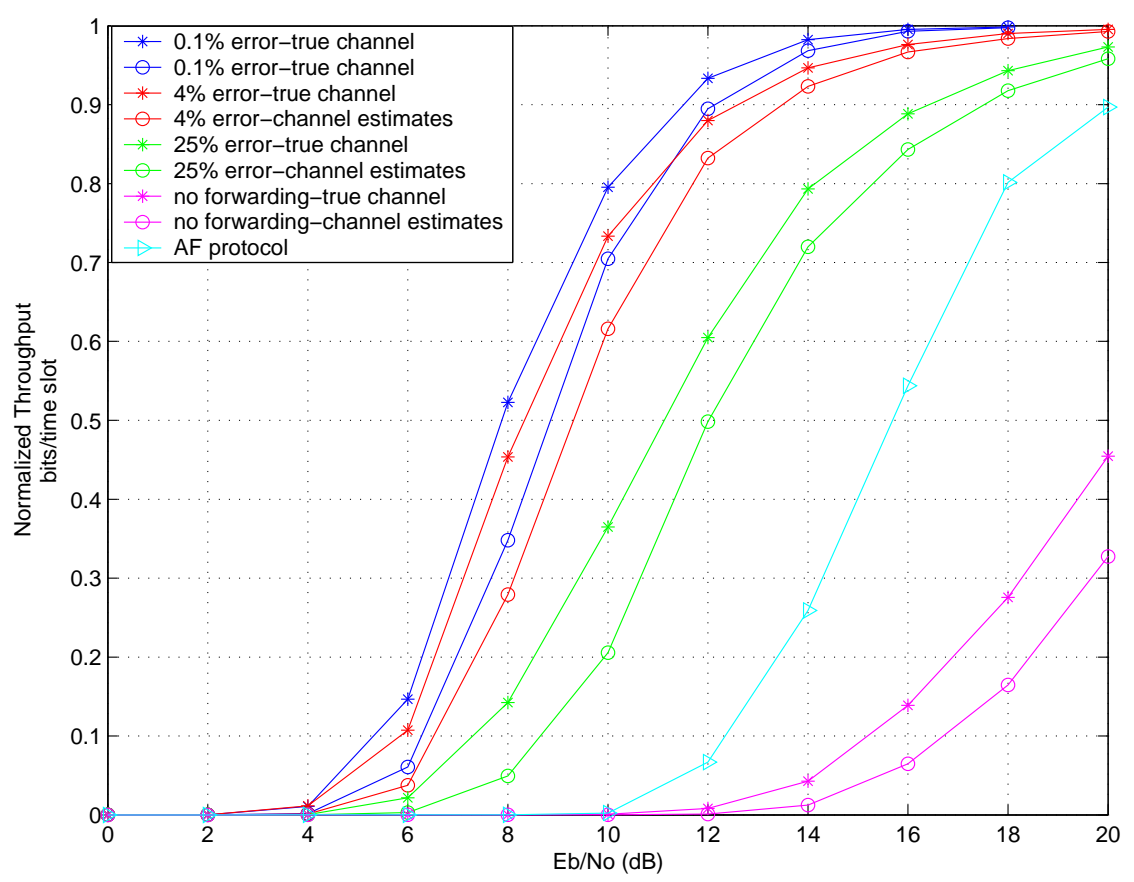

Figure 5.3: Throughput performance of three-user DF sharing in a synchronous uplink. The performance of AF protocol is also included for comparison.

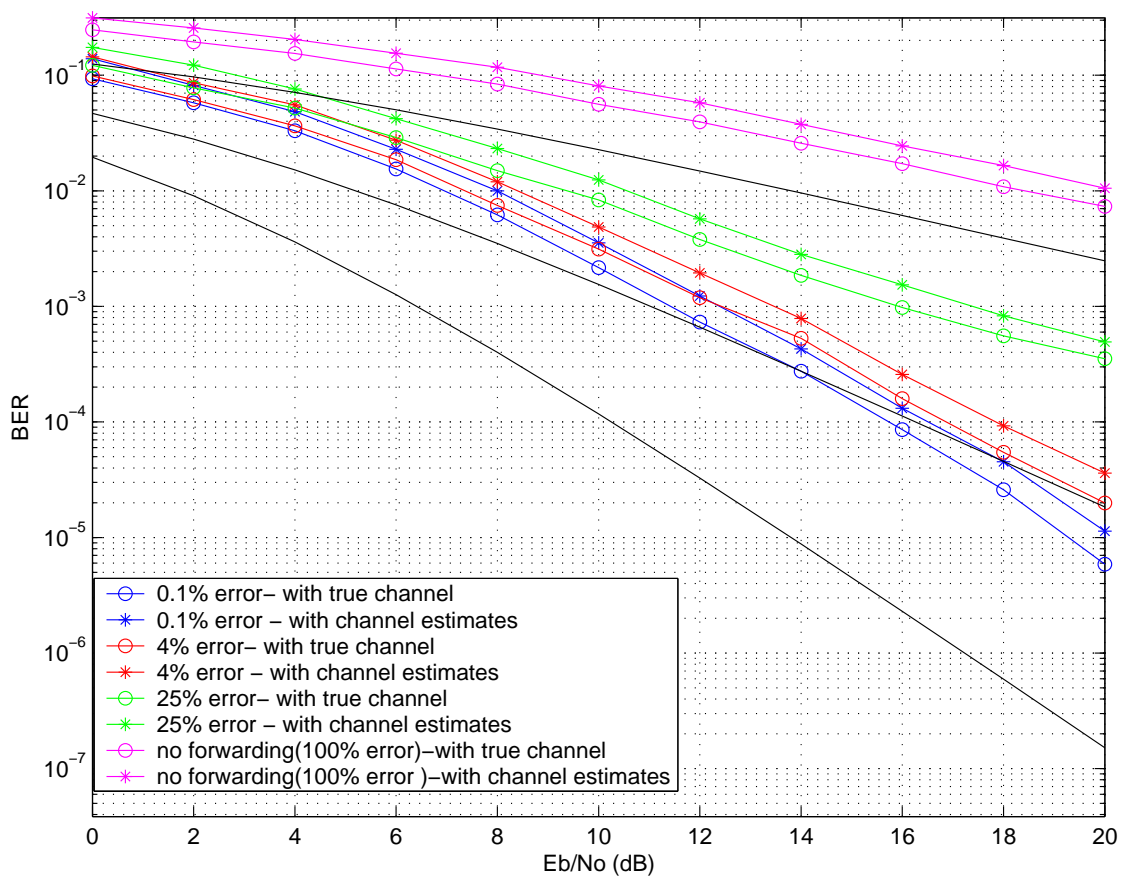

Figure 5.4: BER performance of three-user DF sharing in asynchronous uplink. The performance of maximum-ratio combining with 2 and 3 antennas is also included for comparison. 


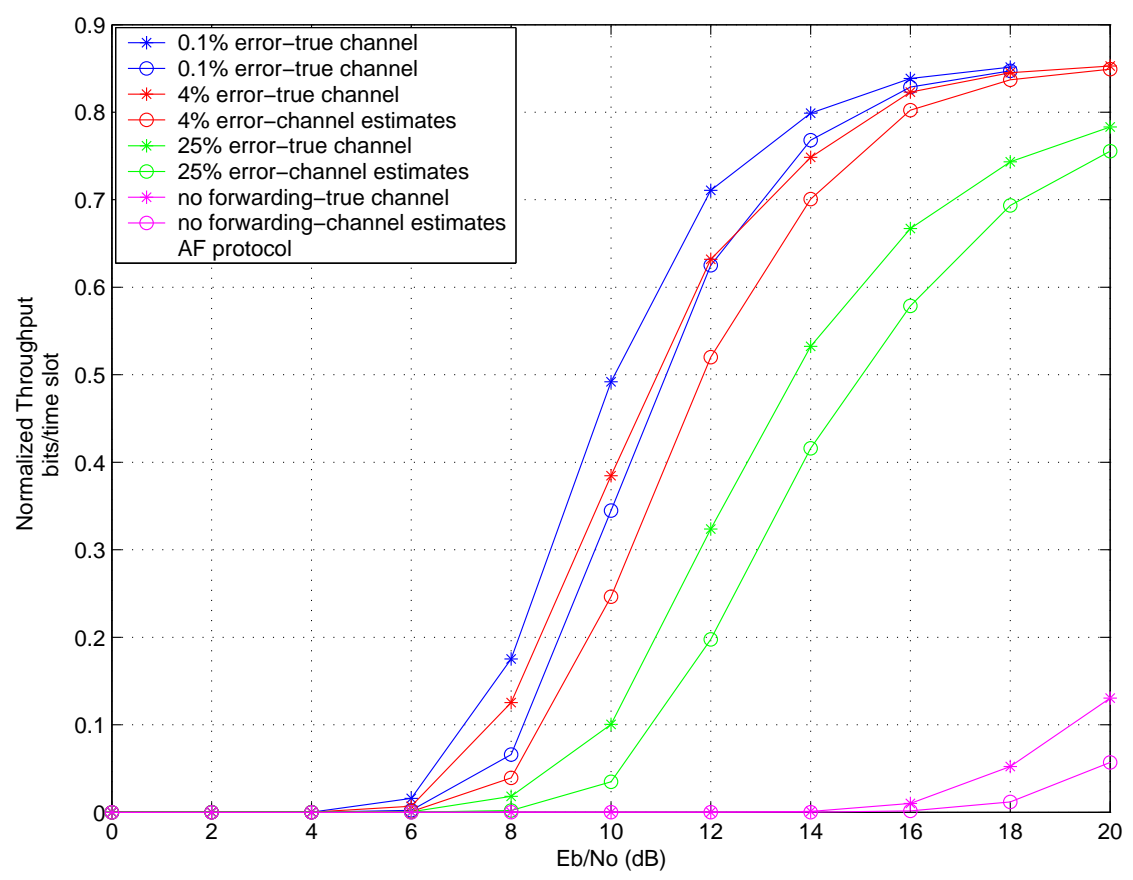

Figure 5.5: Throughput performance of three-user DF sharing in asynchronous uplink.

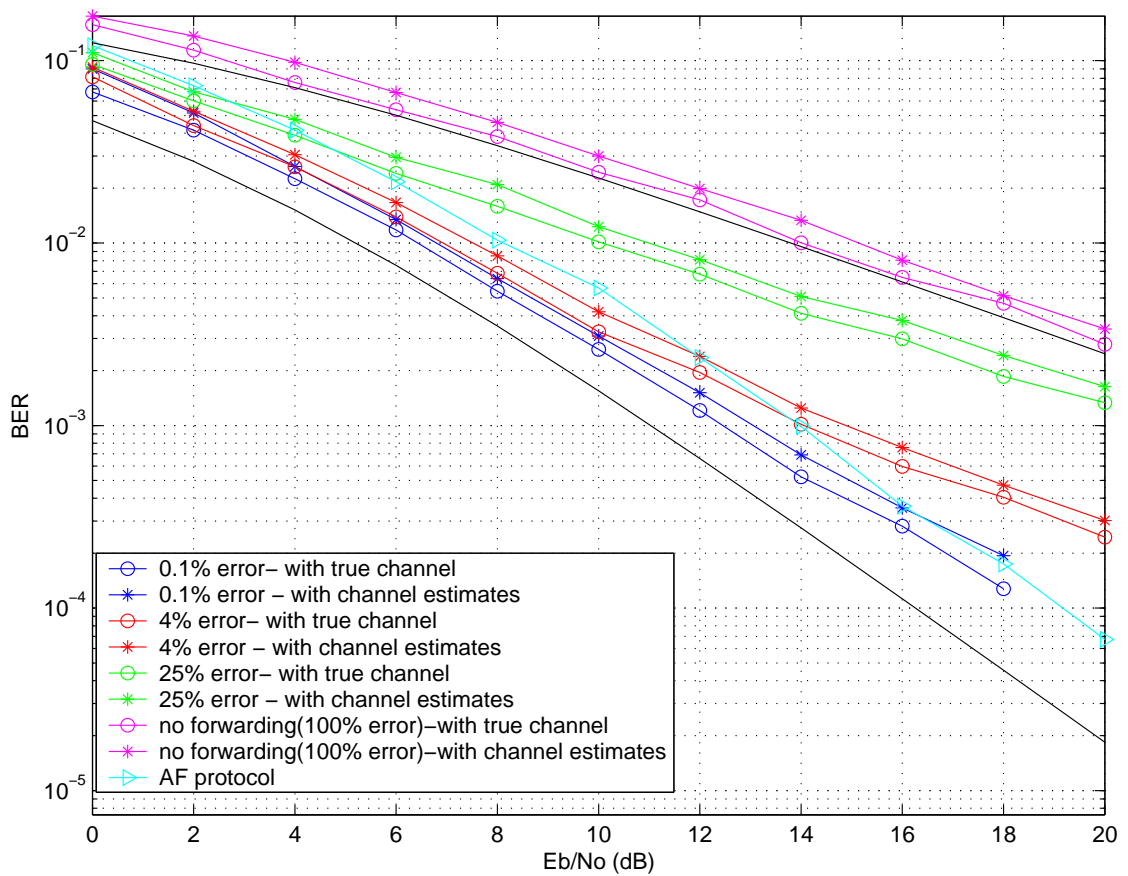

Figure 5.6: BER performance of two-user DF sharing in a synchronous uplink. The performance of maximum-ratio combining with 1 and 2 antennas is also included for comparison. 


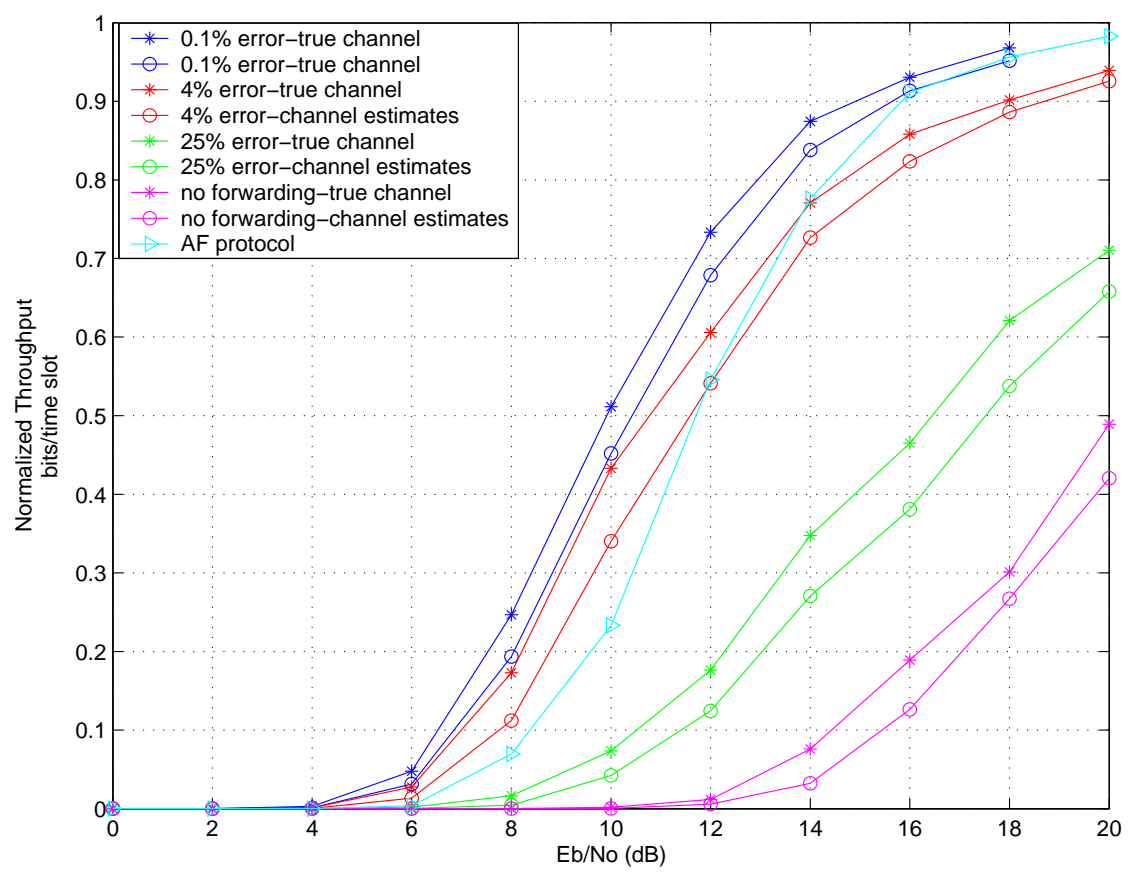

Figure 5.7: Throughput performance of two-user DF sharing in a synchronous uplink. The performance of AF protocol is also included for comparison.

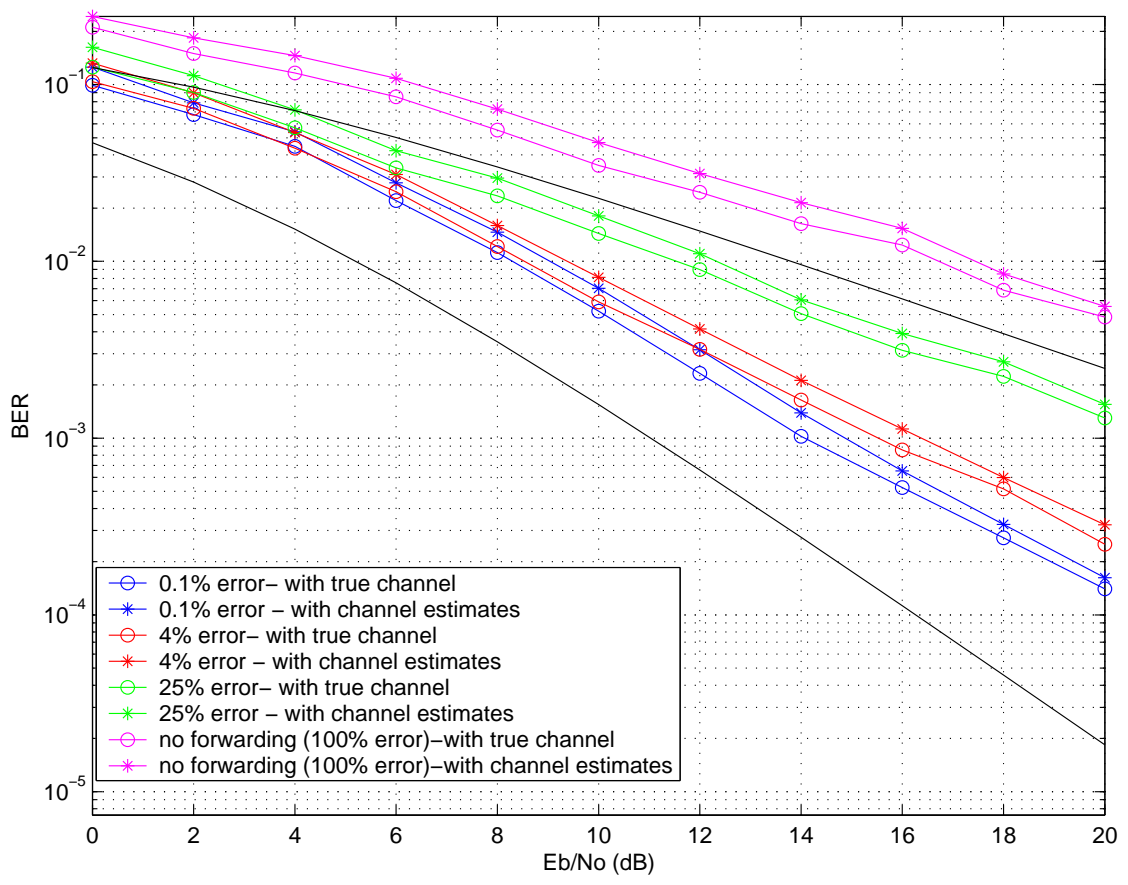

Figure 5.8: BER performance of two-user DF sharing in asynchronous uplink. The performance of maximum-ratio combining with 1 and 2 antennas is also included for comparison. 


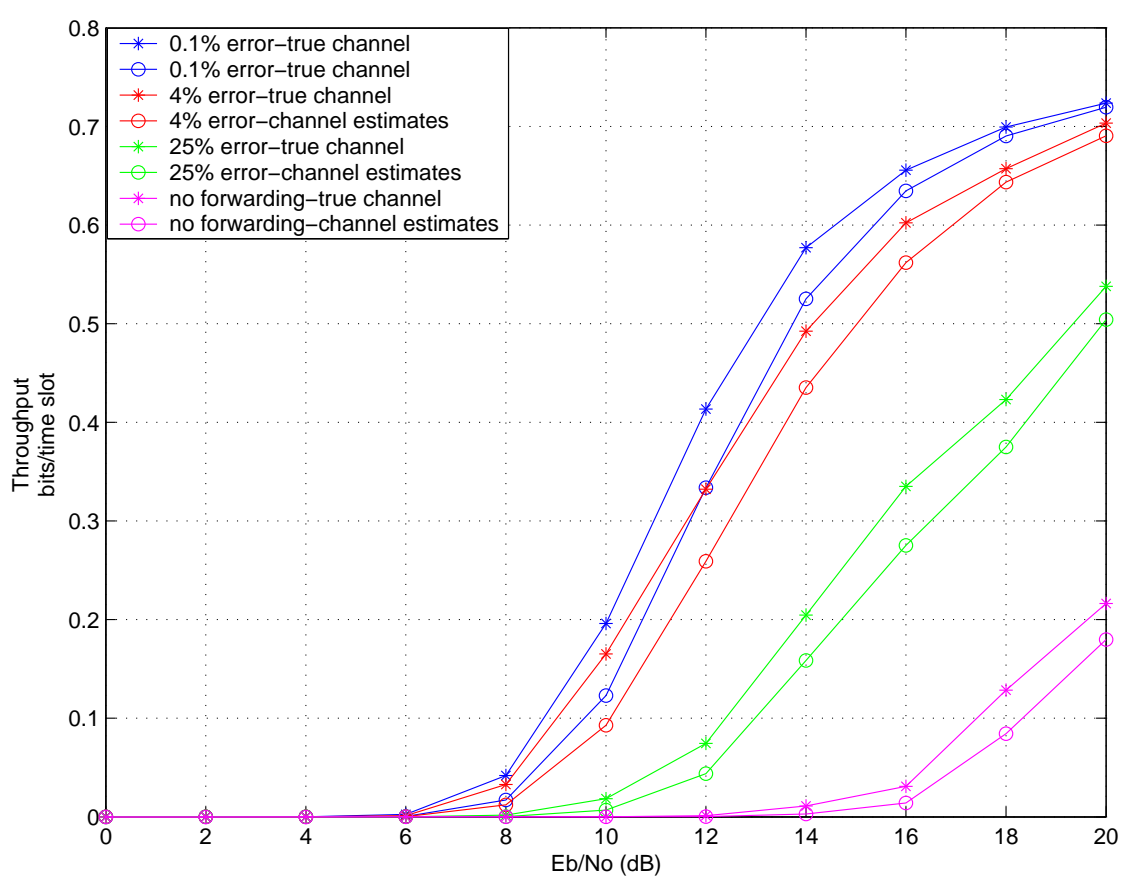

Figure 5.9: Throughput performance of two-user DF sharing in asynchronous uplink.

Fig. 5.2 shows the BER performance of three-user DF protocol operating in a synchronous cellular uplink. The BER curves are plotted for various inter-user channel qualities. It can be seen that when the demodulation error at the cooperating user side is as high as $0.1 \%$, full diversity (MRC-3 performance) can be achieved. $4 \%$ error causes the diversity performance to degrade to MRC-2 performance. When the error is $25 \%$, the three-user sharing scheme still provides diversity gain of 2 but it suffers from an SNR loss as compared to the case when error is 4\%. Performance of three-user AF protocol is also plotted for comparison. It can be seen that AF protocol provides the same diversity gain as DF protocol with $25 \%$ demodulation error, but suffers from a significant SNR loss.

Fig. 5.3 illustrates the normalized throughput performance of the three-user DF protocol in synchronous environments, along with no-sharing. Normalized throughput, $T_{h}$, is calculated using (3.11) with $M=256$ as the packet size. Increasing the packet size here degrades the throughput performance of the system. The protocol operates at full rate $(R$ $=1$ ) in synchronous uplink. AF protocol offers better throughput than only no-sharing case.

Fig.5.4 illustrates the BER performance of three-user sharing scheme in asynchronous 
communication system. It is seen that the diversity performance in this case is the same as that obtained in case of synchronous communication environments except with the SNR loss.

Fig. 5.5 indicates the throughput performance of three-user DF sharing scheme considering in asynchronous communications. The maximum throughput in this case is limited by $6 / 7$ due to zero padding between frames.

Figs. 5.6 and 5.7 show the BER and throughput performance of two-user sharing scheme operating in synchronous manner. For two-user sharing protocol operating in synchronous environments, almost full diversity (MRC-2 performance) is obtained with demodulation errors up to $4 \%$. No diversity gain can be extracted using two-user cooperative diversity sharing protocol if the inter-user channel is bad enough to cause errors $\geq 25 \%$. In contrast to three-user sharing protocol, AF protocol has more diversity gain (diversity order $=2$ ) than the corresponding DF protocol when inter-user channel introduces $4 \%$ demodulation error.

Figs. 5.8 and 5.9 present the BER and throughput performances of two-user sharing in the presence of asynchronism. In this case, two-user protocol operates at $R=3 / 4$. The BER Performance of two-user sharing protocol in asynchronous communications is the same as the corresponding performance in synchronous case in terms of the diversity gain but it shows SNR loss when compared to the synchronous case.

From all the simulations results, it can be seen that as the percentage demodulation error at the cooperating user side increases, two-user and three-user sharing schemes show loss in both the diversity and also SNR loss. It is also seen that by employing batch-adaptive algorithm for channel estimation, little performance degradation in terms of SNR loss (1dB) is incurred as compared to the case where perfect channel estimates are available. But there is no loss in diversity using adaptive receiver design.

\subsection{Conclusion}

Because of random decision errors introduced by cooperating users, channel estimation plays an important role in the receiver design when we consider noisy inter-user channels. It is seen that by employing batch-adaptive algorithm for channel estimation and by using 
these channel estimates to form a detector for joint multiple access interference suppression and space-time decoding, little performance degradation in terms of SNR loss (1 dB loss) is incurred as compared to the case where perfect channel estimates are available. But there is no loss in diversity using the adaptive structure. Also, both two-user and three-user sharing schemes suffer from loss in the diversity order as the percentage error at the mobile units increases. 


\section{Chapter 6}

\section{Information Outage Probability}

\section{Analysis}

In the previous chapters, new cooperative diversity strategies for an asynchronous cellular uplink were presented, along with an appropriate receiver design and simulation results that demonstrated significant throughput performance gains over existing multiuser cooperative strategies. We considered practical cases such as asynchronous communications scenarios and the presence of noisy inter-user channels. In this chapter, we will provide an informationtheoretic outage probability analysis based loosely on the approach in [37].

In [37], the authors analyze repetition-based and space-time coded cooperative diversity using outage probability (where outage probability is the probability that the achievable rate falls below a certain threshold) as a performance measure. One of the limitations of their work is the following. The authors consider a medium access control protocol that requires time orthogonal transmissions between source-destination and relay-destination channels, limiting the bandwidth efficiency. As described in the previous chapters, our system makes use of CDMA to separate symbol streams rather than orthogonal time slots. So the contribution of this chapter is an investigation of which use of bandwidth provides better outage probability. We will see that the proposed sharing schemes which operate in an asynchronous communication environments at full rates offer significantly lower outage probabilities than that have been previously achieved.

\footnotetext{
${ }^{1}$ Part of the work presented in this chapter has been accepted in [46]
} 
Since we are comparing our scheme with the protocols given in [37], we duplicate some of their work in this chapter. We will only present important results for repetition-based and space-time coded protocols from [37] and then we will present the outage probability expression for our space-time coded scheme which we will refer to as multi-user (with three or more users) cooperation scheme. Also, the basic concepts from information theory can be found in Appendix E.

\subsection{Outage Events and Outage Probability}

Consider a single-user wireless system where the transmitter and the receiver has a single antenna. We focus on a scenario in which channel information is available at the receiver but not at the transmitter. The discrete time signal model can be written as

$$
y[i]=\mathbf{a} x[i]+n[i]
$$

where $y[i]$ and $x[i]$ represent the received and the transmitted signals respectively and a is the fading parameter. When the noise $n[i]$ is complex Gaussian with variance $N_{0}$, and $x[i]$ has a power constraint $\mathrm{P}$, the mutual information between the transmitter and the receiver has the form

$$
I=\log \left(1+\frac{|\mathrm{a}|^{2} P}{N_{0}}\right)
$$

As can be observed from the above formula, the average mutual information is a function of fading parameters and hence is a random variable. Thus it is possible that for certain channel realizations, the mutual information is below some fixed required spectral efficiency $R$ i.e.,

$$
\log \left(1+\frac{|\mathrm{a}|^{2} P}{N_{0}}\right)<R
$$

The event $(I<R)$ is referred to as an information outage event. The probability of outage is thus $\operatorname{Pr}[I<R]$. We will use the same notion of outage probability throughout this chapter. 


\subsection{System Model}

Here we briefly mention the system model that is applicable to Laneman's protocols (repetition based protocol and space-time coded protocol) [37], and our proposed sharing schemes (multi-user cooperation schemes). Other design parameters specific to multi-user cooperation schemes will be quoted in the later section. Consider a wireless network with $m$ cooperating terminals where each terminal has information to transmit to the same destination. The analysis provided here mainly deals with decode and forward cooperation. Further details of channel model and SNR parameterizations can be found in [37]. The received signal at any terminal or at the receiver has the form,

$$
y[n]=\mathrm{a}_{i, j} x[n]+z[n]
$$

where $\mathrm{a}_{i, j}$ are zero mean complex Gaussian random variables with variances $1 / \lambda_{i, j}$. We note that the magnitudes $\left|\mathbf{a}_{i, j}\right|$ are Rayleigh distributed and $\left|\mathbf{a}_{i, j}\right|^{2}$ are exponentially distributed with parameter $\lambda_{i, j}$.

\subsection{Laneman's Repetition-Based Cooperative Diver- sity Protocol}

The wireless network considered here consists of $m$ users, each transmitting information to a single destination, using $m-1$ terminals as relays. Due to symmetry of channel allocations, the case of a single source transmitting to a single destination using $m-1$ relays is considered. In Laneman's repetition based protocol, source broadcasts a message. The message is received by the cooperating relays as well as the destination. Relays that could decode the information repeat the source message on orthogonal subchannels. The relays experiencing high mutual information toward source are considered to be decoding relays (i.e., the mutual information between the source and the decoding relays is above some fixed spectral efficiency). Each of the relays here employ the same Gaussian codebook. Hence we have diversity combining effect. Also, transmission between source and destination utilizes a fraction $1 / m$ of the total degrees of freedom ${ }^{2}$ in the channel. The mutual information (in

\footnotetext{
${ }^{2}$ The notion of degrees of freedom is explained in Appendix E.
} 
bits $/ \mathrm{sec} / \mathrm{Hz}$ ) between source and destination conditioned on decoding set $D(s)$ is

$$
I_{\text {rep }}=\frac{1}{m} \log \left(1+\mathrm{SNR}\left|\mathrm{a}_{\mathrm{s}, \mathrm{d}}\right|^{2}+\mathrm{SNR} \sum_{r \in D(s)}\left|\mathrm{a}_{r, d}\right|^{2}\right)
$$

where SNR $=m P_{c} / N_{0} W=P / N_{0}$, where $P_{c}$ is the continuous time power constraint and $W$ is the total bandwidth. The factor $m$ appears in the formulation of SNR since each terminal transmits in fraction $1 / m$ of the available degrees of freedom for repetition-based cooperative diversity. The realized mutual information between source and relay for i.i.d complex Gaussian codebook is

$$
\frac{1}{m} \log \left(1+\operatorname{SNR}\left|\mathrm{a}_{s, r}\right|^{2}\right)
$$

The probability that the mutual information between source and destination falls below some fixed spectral efficiency $R$ (also called as outage probability) is [37]

$$
\operatorname{Pr}\left[I_{r e p}<R\right] \sim\left[\frac{2^{m R}-1}{\mathrm{SNR}}\right]^{m} \times \sum_{D(s)} \lambda_{s, d} \times \prod_{r \in D(s)} \lambda_{r, d} \prod_{r \notin D(s)} \lambda_{s, r} \times \frac{1}{|D(s)+1| !} .
$$

The above expression gives a high SNR approximation to outage probability.

\subsection{Laneman's Space-Time Coded Cooperative Diver- sity Protocol}

Laneman's space-time coded protocol operates in a similar manner as his repetitionbased protocol, except that all relays transmit simultaneously on the same subchannel using a space-time code. Hence this protocol has better bandwidth efficiency than repetitionbased algorithm. Each cooperating terminal transmits in $1 / 2$ the total degrees of freedom in the channel. There are two parallel channels, one from source to destination and another from decoding relays to destination. Hence code combining effect gives mutual information between source and destination conditioned on decoding set $D(s)$ to be

$$
I_{s t c}=\frac{1}{2} \log \left(1+\frac{2}{m} \operatorname{SNR}\left|\mathrm{a}_{s, d}\right|^{2}\right)+\frac{1}{2} \log \left(1+\frac{2}{m} \operatorname{SNR} \sum_{r \in D(s)}\left|\mathrm{a}_{r, d}\right|^{2}\right) .
$$

\footnotetext{
${ }^{3}$ logarithms in this chapter are taken to base 2.
} 
The realized mutual information between source and relay for i.i.d complex Gaussian codebooks is

$$
\frac{1}{2} \log \left(1+\frac{2}{m} \mathrm{SNR}\left|\mathrm{a}_{s, r}\right|^{2}\right)
$$

Using high-SNR approximation, the expression for outage probability is obtained as [37]

$$
\operatorname{Pr}\left[I_{s t c}<R\right] \sim\left[\frac{2^{2 R}-1}{2 \mathrm{SNR} / m}\right]^{m} \times \sum_{D(s)} \lambda_{s, d} \times \prod_{r \in D(s)} \lambda_{r, d} \prod_{r \notin D(s)} \lambda_{s, r} \times A_{|D(s)|}\left(2^{2 R}-1\right)
$$

where

$$
A_{n}(t)=\frac{1}{(n-1) !} \int_{0}^{1} \frac{w^{n-1}(1-w)}{(1+t w)} d w
$$

\subsection{Multi-user Cooperation Scheme}

Our proposed multi-user scheme offers advantages over Laneman's repetition-based and space-time coded protocols because it operates at full rate. The scheme is explained in detail in Chapter 4. CDMA implementation for this scheme allows all cooperating terminals to simultaneously transmit in the same frequency band. Full duplex transmission was also assumed. Here we will see that the performance is only limited by the cross-correlation properties of the spreading codes and that the bandwidth efficiency is superior to Laneman's protocols.

For simplicity, we present a more general signal model. Later we will see that we can extend this to more specific signal model given in Chapter 4. In general, with CDMA implementation, the received signal model (at the cooperating nodes and also at the destination) can be written as

$$
\boldsymbol{r}=\boldsymbol{H} \boldsymbol{s}+\boldsymbol{n}
$$

where $\boldsymbol{r}$ is the received signal vector, $\boldsymbol{H}$ a function of spreading codes and channel gains is the effective channel matrix, $\boldsymbol{s}$ is a symbol vector and $\boldsymbol{n} \sim \mathcal{N}_{c}\left(0, \sigma^{2} \boldsymbol{I}\right)$. This can further be written as

$$
\boldsymbol{r}=\tilde{\boldsymbol{H}} \boldsymbol{\alpha} \boldsymbol{s}+\boldsymbol{n}
$$


where $\tilde{\boldsymbol{H}}$ is a function of spreading codes and $\boldsymbol{\alpha}$ is a structured matrix where each row has only one non-zero element (typically it is a diagonal matrix) and is a function of only channel gains. We will show in the Appendix F that our specific three-user sharing scheme signal model can also be represented as (6.13). We apply decorrelating multi-user detector, so that

$$
\begin{aligned}
\boldsymbol{r}_{1} & =\tilde{\boldsymbol{H}}^{H} \boldsymbol{r} \\
& =\underbrace{\tilde{\boldsymbol{H}}^{H} \tilde{\boldsymbol{H}}}_{\boldsymbol{R}} \boldsymbol{\alpha} \boldsymbol{s}+\tilde{\boldsymbol{H}} \boldsymbol{n}
\end{aligned}
$$

Then,

$$
\begin{aligned}
\boldsymbol{d} & =\boldsymbol{R}^{-1} \boldsymbol{r}_{1} \\
& =\boldsymbol{\alpha} \boldsymbol{s}+\boldsymbol{v}
\end{aligned}
$$

where $\boldsymbol{v} \sim \mathcal{N}_{c}\left(0, \sigma^{2} \boldsymbol{R}^{-1}\right)$. Since $\boldsymbol{\alpha}$ is a special structure matrix with exactly one non-zero term in each row, denoting these terms by $a_{i, j}$, we can split the above matrix-vector model into separate linear equations of the form

$$
\boldsymbol{d}[i]=\mathrm{a}_{i, j} \boldsymbol{s}[i]+\boldsymbol{v}[i]
$$

Thus the specified use of decorrelating multiuser detection at the base station effectively transforms the resulting vector channel into $\mathrm{K}$ (the number of users) parallel scalar AWGN channels, where the MAI and ISI is manifested as increased background noise [51]. Note that the cooperating terminals utilize all the available degrees of freedom in the channel. Because of the decorrelation process, the effective SNR is now SNR $/ \boldsymbol{R}^{-1}$. Hence the mutual information between source and destination conditioned on decoding set can be given by

$$
I_{\text {multi-user }}=\log \left(1+\frac{\mathrm{SNR}}{m\left[\boldsymbol{R}^{-1}\right]_{1,1}}\left|\mathrm{a}_{s, d}\right|^{2}\right)+\log \left(1+\frac{\mathrm{SNR}}{m} \sum_{i=r \in D(s)} \frac{\left|\mathrm{a}_{r, d}\right|^{2}}{\left[\boldsymbol{R}^{-1}\right]_{i, i}}\right) .
$$

The realized mutual information between source and relay has the form

$$
\log \left(1+\frac{\mathrm{SNR}}{m\left[\boldsymbol{R}^{-1}\right]_{s, r}}\left|\mathrm{a}_{s, r}\right|^{2}\right) .
$$

The decoding set probability is given by

$$
\operatorname{Pr}[D(s)] \sim\left[\frac{2^{R}-1}{\mathrm{SNR} / m}\right]^{m-D(s)-1} \times \prod_{r \notin D(s)} \lambda_{s, r} .
$$


Again, using high-SNR approximation, we can write an expression for outage probability as

$$
\begin{aligned}
\operatorname{Pr}\left[I_{\text {multi-user }}<R\right] \sim & {\left[\frac{2^{R}-1}{\operatorname{SNR} / m}\right]^{m} \times \sum_{D(s)} \lambda_{s, d}\left[\boldsymbol{R}^{-1}\right]_{1,1} \times \prod_{i=r \in D(s)} \lambda_{r, d}\left[\boldsymbol{R}^{-1}\right]_{i, i} } \\
& \times \prod_{j=r \notin D(s)} \lambda_{s, r}\left[\boldsymbol{R}^{-1}\right]_{j, j} \times A_{|D(s)|}\left(2^{R}-1\right)
\end{aligned}
$$

To arrive at the above result, we follow the similar procedure as given in [52] with some modifications, which we describe here. In addition to the analytical results discussed in [37], we make use of the following identities while deriving (6.22).

- Let $u_{k}, k=1,2, \ldots, m$ be positive, independent exponential random variables with

$$
\liminf _{\epsilon \longrightarrow 0} p_{u_{k}}(\epsilon u) \geq \lambda_{k}
$$

where $\lambda_{k}$ 's are the parameters.

and suppose we have $t_{k}=\frac{u_{k}}{c_{k}}, c_{k}$ 's being some constant. Then

$$
\liminf _{\epsilon \longrightarrow 0} p_{t_{k}}(\epsilon t) \geq c_{k} \lambda_{k}
$$

- If $t_{k}, k=1,2, \ldots, m$ be positive, independent random variables with

$$
\liminf _{\epsilon \longrightarrow 0} p_{t_{k}}(\epsilon t) \geq c_{k} \lambda_{k}
$$

Then

$$
\lim _{\epsilon \longrightarrow 0} \frac{1}{\epsilon^{m}} \operatorname{Pr}\left[\sum_{k=1}^{m} t_{k}<\epsilon\right]=\frac{1}{m !} \prod_{k=1}^{m} \lambda_{k} c_{k}
$$

The proof of (6.22) is akin to [37]. A sketch of a proof appears in the Appendix G.

Observe that the $\left[\boldsymbol{R}^{-1}\right]_{k, k}$ factors in the final expression of outage probability indicate the effect of multiple access interference. As the spreading codes of large cross correlation values are assigned to users, the performance becomes worse. But these terms occur inside the log function in the expression for mutual information, while in case of Laneman's protocols, the penalty (due to the fraction of total degrees of freedom in the channel occupied by the cooperating terminals ) accours outside the log function in the expression for mutual information. We will see that with moderate cross-correlation values of spreading codes, the multi-user cooperation schemes outperform Laneman's protocols in terms of the outage probabilities. 


\subsection{Sendonaris's Cooperation Scheme}

We also compare outage probability expression for multi-user cooperation scheme with the existing two-user cooperation scheme explained in [5]. Some of the limitations of Sendonaris's cooperation protocols are: (a) It is a two-user cooperation protocol with CDMA implementation and is not generalizable to more number of cooperating users. (b) Authors assume orthogonal spreading codes and synchronous communication which are not practical to achieve. In Chapter 4, we compared our two-user sharing scheme with Sendonaris's two-user protocol taking into account the bit-error-rate and the throughput as performance measures. In this section, we extend the comparison considering outage probability as a performance measure. Since there are variations of Sendonaris's protocols, for comparison purposes, we consider rate-half cooperation scheme.

\subsubsection{Case I}

If we consider, exactly the same assumptions of orthogonal codes and synchronous communication as stated in [5] and the half rate version of Sendonaris's protocol (for which we have also presented BER and throughput results in previous chapters), we can say that cooperating terminals transmit in half the available degrees of freedom. Hence, in this case, we get exactly the same outage probability expression as in the case of Laneman's space-time coded cooperative diversity protocol.

$$
\operatorname{Pr}\left[I_{\text {Case-I }}<R\right] \sim\left[\frac{2^{2 R}-1}{2 \mathrm{SNR} / m}\right]^{m} \times \sum_{D(s)} \lambda_{s, d} \times \prod_{r \in D(s)} \lambda_{r, d} \prod_{r \notin D(s)} \lambda_{s, r} \times A_{|D(s)|}\left(2^{2 R}-1\right)
$$

The cooperation protocol given in [5] is a half duplex protocol and assumptions of synchronous communication in addition to orthogonal spreading codes eliminates the multipleaccess interference and hence it is very similar to transmitting in different subchannels as in [37]. We can say that Laneman's space-time coded scheme with $m=2$ and Sendonaris's twouser cooperation scheme are equivalent because eventually terminals occupy same number of degrees of freedom in the system. 


\subsubsection{Case II}

Here, we consider a two-user scheme given in [5] under more realistic assumption of asynchronous communication and/or non-orthogonal spreading codes. If we assume asynchrnous communications, then even orthogonal spreading codes lose their orthogonality. Thus the assumption of asynchronous communication indirectly takes into account the consequence of using non-orthogonal spreading codes. The result is the presence of multiple-access interference. The signal model is now the same as presented in the last section where $\boldsymbol{R}^{-1}$ terms indicate the effect of multiple access interference. The expression for outage probability in this case can be expressed as

$$
\begin{aligned}
\operatorname{Pr}\left[I_{\text {Case-II }}<R\right] \sim & {\left[\frac{2^{2 R}-1}{2 \mathrm{SNR} / m}\right]^{m} \times \sum_{D(s)} \lambda_{s, d}\left[\boldsymbol{R}^{-1}\right]_{1,1} \times \prod_{i=r \in D(s)} \lambda_{r, d}\left[\boldsymbol{R}^{-1}\right]_{i, i} } \\
& \times \prod_{j=r \notin D(s)} \lambda_{s, r}\left[\boldsymbol{R}^{-1}\right]_{j, j} \times A_{|D(s)|}\left(2^{2 R}-1\right)
\end{aligned}
$$

Again, as stated in the last section, $\boldsymbol{R}^{-1}$ has a detrimental effect on the outage probability performance of the system.

\subsection{Results}

We plot outage probability curves taking into account spreading codes with various crosscorrelation values. To provide baseline performance comparison results, we consider correlation matrix $\boldsymbol{R}$ as having diagonal values equal to unity and off-diagonal elements being equal to $\rho$.

It can be seen that the outage probability performance of multi-user sharing scheme degrades as the spreading codes with large cross-correlation properties are assigned to users. But with moderate cross-correlation values of spreading codes, multi-user sharing scheme offers better performance than Laneman's repetition-based and space-time coded protocols in terms of the outage probability. Note that, since our distributed multi-user cooperation scheme is based upon CDMA implementation and also operates at full rate, performance is only limited by multiple-access interference. We observe that the effective SNR for multi-user cooperation scheme is $\operatorname{SNR} /\left[\boldsymbol{R}^{-1}\right]$. The $\operatorname{SNR}($ effective) term appears inside the $\log (\cdot)$ function 
of mutual information expression. In Laneman's protocols, since transmission between source and destination does not utilize all available degrees of freedom in the channel, the penalty occurs outside the $\log (\cdot)$ function in the formula for mutual information which has prominent degradation effect on the outage probability performance as compared to multi-user cooperation scheme.

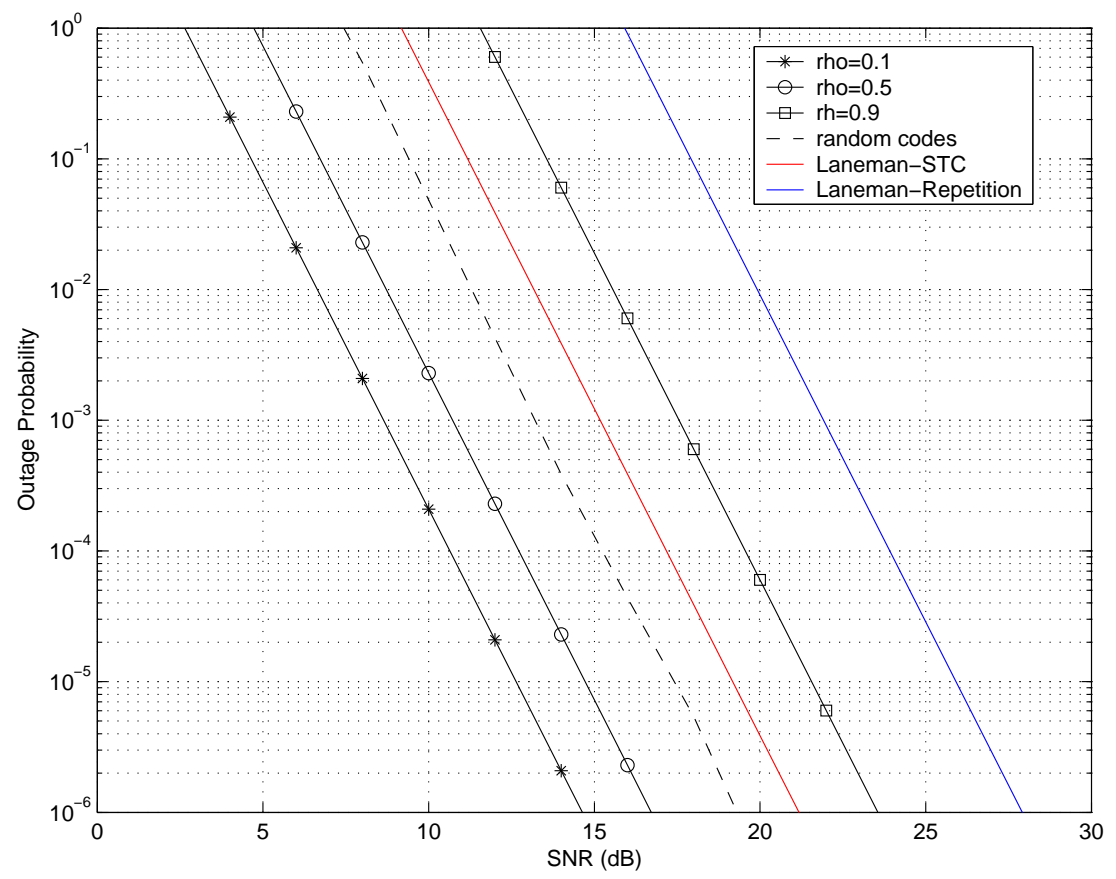

Figure 6.1: Outage probability performance of multi-user cooperation scheme for network size of $\mathrm{m}$ (number of users) $=5$. 'rho' indicates the cross-correlation between spreading codes. Outage probability for Laneman's repetition-based and space-time coded cooperative diversity protocols is also plotted for comparison for same network size.

Outage probability performance for various network sizes and $\rho=0.7$ is plotted in Fig.6.2. The performance of multi-user cooperation protocol with $\rho=0.7$ is very close to that obtained by using random codes, hence we chose $\rho=0.7$ for plotting outage probability results in Fig. 6.2.

Also, it can be seen that multi-user cooperation scheme shows significant performance improvement over Laneman's space-time coded scheme as number of cooperating terminals increases. Fig. 6.3 compares performance of multi-user cooperation scheme (with $m=2$ ) with Sendonaris's two-user sharing scheme in the presence of multiple-access interference. Multi-user cooperation scheme shows performance improvement in terms of the outage prob- 


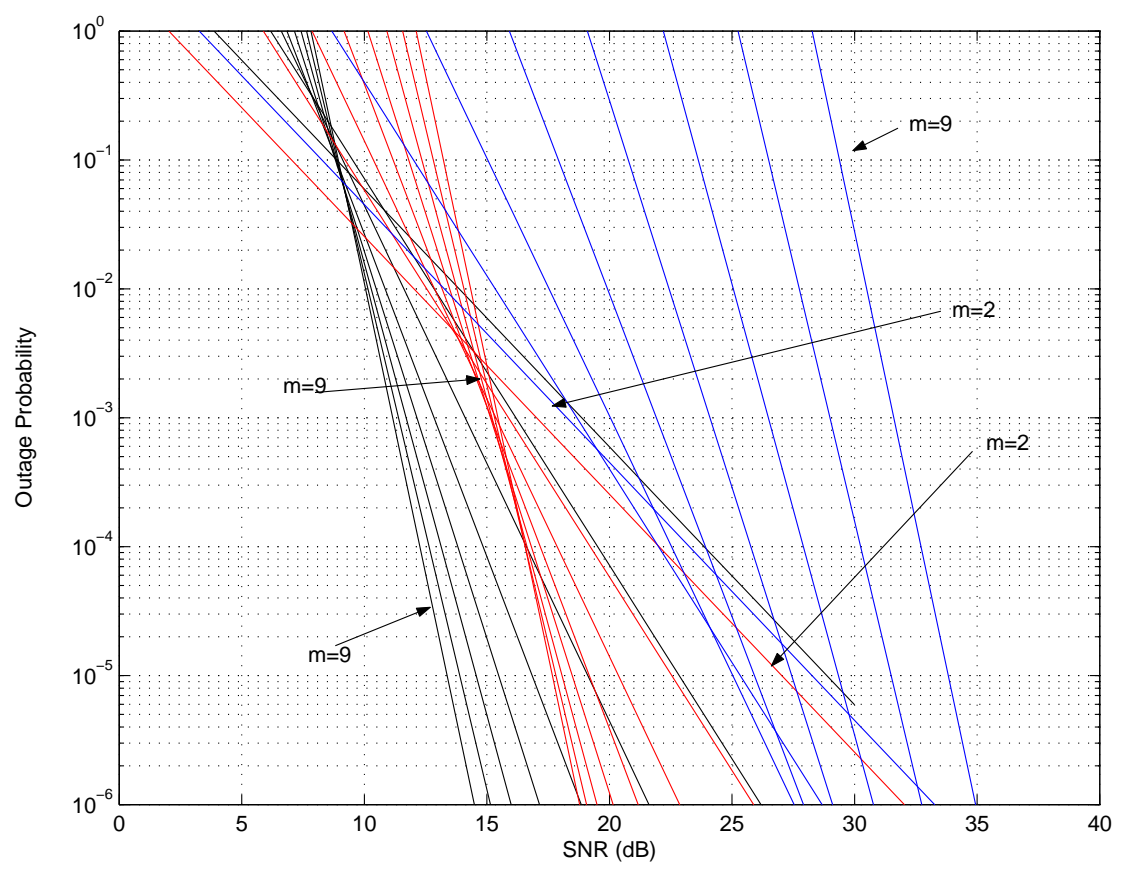

Figure 6.2: Outage probability performance of multi-user cooperation scheme (black color) for network size of $m=2,3, \cdots, 9$ and $\rho=0.7$. Outage probability for Laneman's repetitionbased (blue color) and space-time coded (red color) cooperative diversity protocols is also plotted for comparison for same network size.

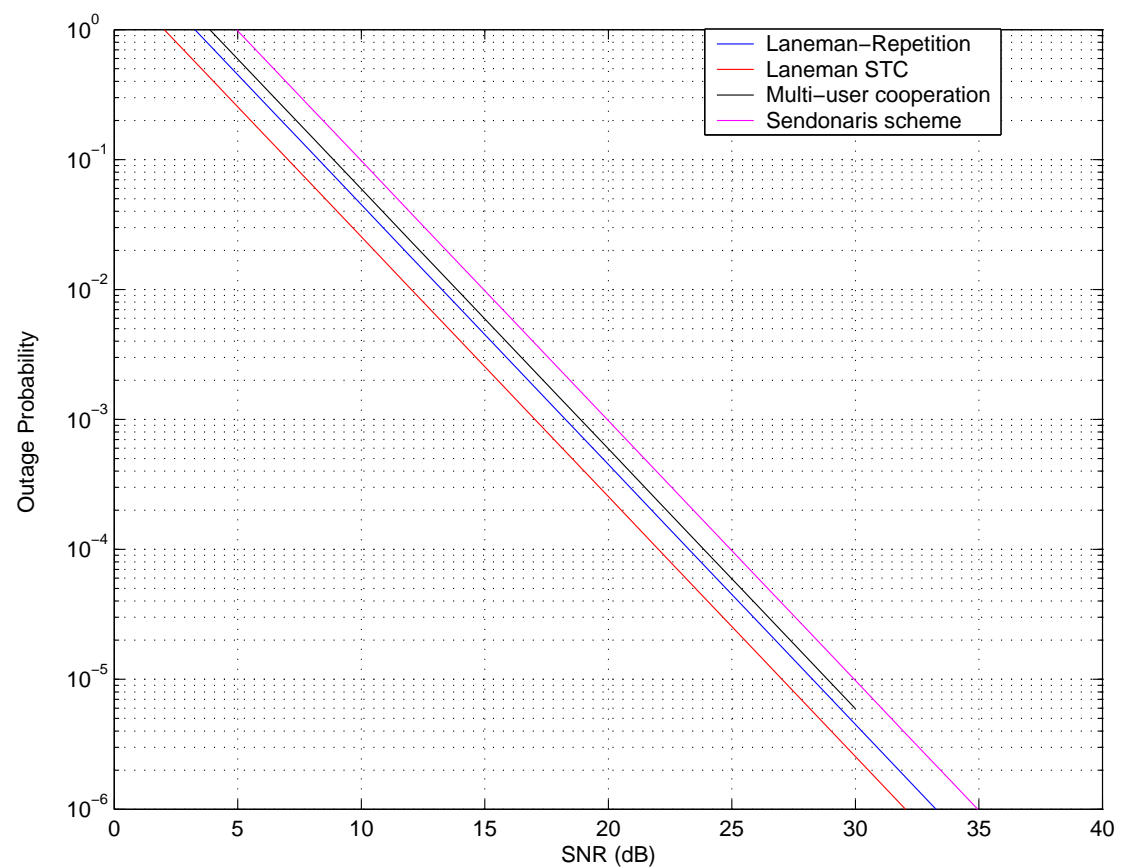

Figure 6.3: Outage probability performance comparison of multi-user cooperation scheme for network size of $m=2$, and Sendonaris's cooperation scheme. 
ability when compared to existing two-user scheme.

\subsection{Conclusion}

Using the scalar channel model with an appropriate SNR parameterizations, the proposed multi-user cooperation schemes can be fairly compared to [37] via outage probability, i.e., the probability that the achievable rate (average mutual information) falls below a given threshold. It is seen that the performance of multi-user scheme is better than existing spacetime coded protocol and is limited only by multiple-access interference. 


\section{Chapter 7}

\section{Conclusions}

\subsection{Summary and Conclusions}

In this thesis, we have developed multi-user sharing strategies for cooperative diversity in a cellular uplink. We considered two-user and three-user decode and forward cooperation schemes, amplify and forward protocol in asynchronous communication environments. Our three-user sharing protocol can also be generalized to a scheme with more cooperating users by incorporating orthogonal or non-orthogonal space-time codes. Both schemes provided near ML performance with a linear MMSE receiver designed for joint multiuser detection and space-time decoding. We considered two scenarios. In the first case, inter-user channels were assumed to be perfect. The assumption of no inter-user errors provided baseline results (upper bounds) for the two-user and three-user sharing schemes. Simulation results showed the significant improvement in the bit-error-rate and throughput performance of the system over existing user cooperation protocols. We also presented simulation results for the amplify and forward protocol for comparison. DF protocol was observed to behave uniformly better than the corresponding AF protocol under the assumption of perfect power control. We further modified our system model to account for the noisy inter-user channels. An adaptive receiver structure was developed to assist the base station in estimating the effective channel matrix, because it is not possible for a base station to gain knowledge of inter-user channel gains. We note here that the specified channel estimator does not require separate estimation of channel gains and delays. We tested the system employing multi-user sharing schemes with 
various inter-user channel qualities. Simulation results showed increase in the SNR loss and the loss in diversity gain with decrease in the inter-user channel quality. Additional expected SNR loss was observed due to channel estimation technique employed at the base station but without any change in the diversity gain. An information-theoretic outage probability analysis for multi-user cooperation protocols was provided, and these protocols were shown to outperform existing space-time coded protocols.

Thus, from our results, we can conclude that user cooperation can improve uplink performance as long as the inter-user channels are good. If the inter-user channel quality is poor, the benefits from cooperation are limited. In addition, it appears that increasing the size of the cooperating group makes the system more sensitive to the inter-user channels. The sharing schemes provide the same diversity order in asynchronous communication environments as that obtained under the assumption of synchronous communication scenarios. Therefore asynchronous communications affect the system performance (BER performance) in terms of the SNR loss, while retaining the diversity gain. The cross-correlation properties of the spreading codes play a crucial role in determining the outage probability performance of full rate sharing schemes operating in a CDMA uplink.

\section{2 $\quad$ Future Work}

In this thesis, we assumed perfect power control, i.e we assumed channels between users and a base station to be statistically identical. Future research work can consider adaptive power control techniques in which the effect of user-base station link quality on the system performance could be studied. Hybrid cooperation techniques could be studied that will allow fraction of the cooperating users to employ amplify and forward protocol while the remaining cooperating users employ the decode and forward protocol. Blind adaptive receiver structure could also be developed for sharing schemes with imperfect inter-user channels. The issue of optimal grouping of users and optimal power allocation need to be investigated. The optimal power allocation schemes would help us decide how much power should we use for relaying and how much for initial transmission. For example, a special case of the power allocation issue is, when do we relay and when do we not relay. 


\section{References}

[1] Y. Hua, Y. Mei and Y. Cheng, "Wireless antennas - making wireless communication perform like wireline communication," in IEEE Topical Conf. on Wireless Commun. Tech., Honolulu, Hawaii, Oct. 2003, pp. 47-73.

[2] N. Laneman, D. Tse, and G. Wornell,, "Cooperative diversity in wireless networks: efficient protocols and outage behavior," IEEE Trans. Inform. Theory, vol. 50, no. 12, pp. 3062-3080, Dec. 2004.

[3] H. El Gamal K. Azarian and P. Schniter, On the achievable diversity-multiplexing tradeoff in half-duplex cooperative channels, available at http://www.ece.osu.edu/ hlga$\mathrm{mal} /$ draft11.pdf.

[4] L. Zheng, D. Tse, "Diversity and multiplexing: A fundamental tradeoff in multiple antenna channels," IEEE Trans. Inform. Theory, vol. 49, pp. 1073-1096, May 2003.

[5] A. Sendonaris, E. Erkip, B. Aazang, "User cooperative diversity - Part I: System description," IEEE Trans. Commun., vol. 51, no. 1, pp. 1927-1938, Nov. 2003.

[6] A. Sendonaris, E. Erkip, B. Aazang, "User cooperative diversity - Part II: Implementation aspects and performance analysis," IEEE Trans. Commun., vol. 51, no. 1, pp. 1939-1948, Nov. 2003.

[7] A. Stefanov and E. Erkip , "Cooperative space time coding for wireless networks," in IEEE Info. Theory Workshop (ITW), Paris, France, April 2003.

[8] A. Stefanov and E. Erkip , "On the performance analysis of cooperative space time coded systems," in Proc. IEEE Wireless Commun. Network. Conf. (WCNC), March 2003.

[9] J.G. Proakis, Digital Communications, 3rd ed., New York, NY: McGraw-Hill, 1995.

[10] A. Papoulis, S. Plillai, Probability, Random Varibales and Stochastic Processes, 4th ed., New York, NY: McGraw-Hill, 2002.

[11] B. Woerner, "Communications theory," Course Notes, WVU, Spring 2005.

[12] T. Rappaport, Wireless Communication Systems, 3rd ed., New York, NY: McGraw-Hill, 1995. 
[13] M. Valenti, "Wireless networking," Course Notes, WVU, Fall 2004.

[14] B. Sklar, "Rayleigh fading Channels in mobile digital communication systems. Part-I : characterization," IEEE Commun. Magazine, vol. 35, no. 9, pp. 136-146, Sept. 1997.

[15] D. Reynolds, "Wireless communication systems," Course Notes, WVU, Fall 2003.

[16] A. Paulraj, D. Gore, R. Nabar, H. Bölcskei, "An overview of MIMO communications A key to gigabit wireless," Proc. IEEE, vol. 92, no. 2, pp. 198-218, Feb. 2004.

[17] İ. Telatar, "Capacity of multi-antenna gaussian channels," European Trans. on Telecommun., vol. 10, no. 6, pp. 585-595, Nov. 1999.

[18] T. Marzetta, B. Hochwald, "Capacity of mobile multiple-antenna communication link in a rayleigh flat fading environment," IEEE Trans. Inform. Theory, vol. 45, no. 1, pp. 139-157, Jan. 1999.

[19] G. Foschini and M. Gans, "On the Limits of wireless communications in a fading environment when using multiple antennas," Wireless Personal Commun., vol. 6, no. 3, pp. 311-335, 1998.

[20] L. Zheng, Diversity-Multiplexing Tradeoff: A Comprehensive View of Multiple Antenna Systems, Ph.D. thesis, University of California at Berkeley, Fall 2002.

[21] I. Berenguer, X. Wang, "Space-time coding and signal processing for MIMO communications," Journal of Computer Science and Technology, vol. 18, no. 6, pp. 689-702, Nov. 2003.

[22] G. Foschini, "Layered space-time architecture for wireless communication in a fading environment when using multi-element antennas.," Bell Labs. Tech. Journal, vol. 1, no. 2, pp. 41-59, Autumn 1996.

[23] P. Wolniansky, G. Roschini, G. Golden, and R. Valenzuela, "V-BLAST: an architecture for realizing very high data rates over the rich-scattering wireless channel," in Int. Symp. Sig. Sys. Elect., Pisa, Italy, Sep. 1998.

[24] S. Alamouti, "A simple transmit diversity technique for wireless communications," IEEE J. Select. Areas Commun., vol. 16, no. 8, pp. 1451-1458, Oct. 1998.

[25] V. Tarokh, H. Jafarkhami, and A. R. Calderbank, "Space-time block codes from orthogonal designs," IEEE Trans. Inform. Theory, vol. 45, no. 5, pp. 1456-1467, July 1999.

[26] V. Tarokh, H. Jafarkhami, and A. R. Calderbank, "Space-time block coding for wireless communications: Performance results," IEEE Trans. Inform. Theory, vol. 17, no. 3, pp. 451-460, Mar. 1999.

[27] V. Tarokh, N. Seshadri, and A. R. Calderbank, "Space-time codes for high data rate wireless communications: Performance criterion and code construction.," IEEE Trans. Inform. Theory, vol. 44, no. 2, pp. 744-765, Mar. 1998. 
[28] Y. Hua, Y. Mei, and Y. Chang, "Parallel mobile wireless relays with space-time modulation," in Proc. IEEE Workshop on Statistical Signal Processing, St Louis, MO, Oct. 2003, p. 4773.

[29] Y. Chang and Y. Hua, "Diversity analysis of orthogonal space-time modulation for wireless relays," in Proc. Int. Conf. on Acoustics, Speech and Signal Processing (ICASSP), Montreal, Canada, May 2004, vol. 4, pp. IV561-IV564.

[30] H. Zheng, Y. Zhu, C. Shen, and X. Wang, "On the effectiveness of cooperative diversity in Ad Hoc networks: a MAC layer study," in Proc. Int. Conf. on Acoustics, Speech and Signal Processing (ICASSP), Philadelphia, PA, March 2005.

[31] M. Khojastepour, A. Sabharwal, and B. Aazhang, "On the capacity of 'cheap' relay networks," in Conf. on Inform. Sciences and Sys. (CISS), Baltimore, MD, Apr. 2003.

[32] A. Høst-Madsen, "On the capacity of wireless relaying," in Proc. IEEE Veh. Tech. Conf. (VTC), Vancouver, BC, Sept. 2002, vol. 3, pp. 1333-1337.

[33] S. Barbarossa and G. Scutari, "Distributed space-time coding strategies for wideband multihop networks: regenerative vs. non-regenerative relays," in Proc. Int. Conf. on Acoustics, Speech and Signal Processing (ICASSP), Montreal, Canada, May 2004.

[34] A. Stefanov and E. Erkip, "Cooperative coding for wireless networks," IEEE Trans. Commun., vol. 52, no. 9, pp. 14701476, Sept. 2004.

[35] T. E. Hunter and A. Nosratinia, "Cooperation diversity through coding," in Proc. IEEE Int. Symp. on Inform. Theory (ISIT), Lausanne, Switzerland, July 2002, p. 220.

[36] M. Janani, A. Hedayat, T. Hunter, and A. Nosratinia, "Coded Cooperation in Wireless Communications: Space-time transmission and iterative decoding," IEEE Trans. Signal Processing, vol. 52, no. 2, pp. 362-371, Feb. 2004.

[37] N. Laneman and G. Wornell, "Distributed space time coded protocols for exploiting cooperative diversity in wireless networks," IEEE Trans. Inform. Theory, vol. 49, no. 10, pp. 2415-2425, Oct. 2003.

[38] P. Tarasak, H Minn, Member, and V Bhargava, "Differential modulation for two-user cooperative diversity systems," IEEE J. Select. Areas Commun., vol. 23, no. 9, pp. 1891-1900, Sep. 2005.

[39] A. Goldsmith, S. Wicker, "Design challenges for energy-constrained ad hoc wireless networks," IEEE Wireless Communications, vol. 9, no. 4, pp. 8-27, Aug. 2002.

[40] Y. Lin, Y. Hsu , "Multihop cellular: A new architecture for wireless communication," in IEEE INFOCOM, Mar. 2000, vol. 3, pp. 1273-1282.

[41] P. Jung, P. Baier, Andreas Steil, "Advantages of CDMA and Spread Spectrum Techniques over FDMA and TDMA in Cellular Mobile Radio Applications," IEEE Trans. Veh. Tech., vol. 42, no. 3, pp. 357-364, Aug. 1993. 
[42] B. Zhao, Multiterminal Relay Networks: Performance bounds, protocol design and channel coding strategies, Ph.D. thesis, West Virginia University, Morgantown, WV, 2004.

[43] D. Goeckel and Y. Hao , "Macroscopic Space-Time Coding: Motivation, Performance Criteria, and a Class of Orthogonal Designs," in Conf. on Inform. Sciences and Sys. (CISS), Johns Hopkins University, Mar. 2003, vol. 3.

[44] A. Boariu, and D. M. Ionescu, "A class of nonorthogonal rate-one space time block codes with controlled interference," IEEE Trans. Wireless Comm., vol. 2, no. 2, pp. 270-276, Mar. 2003.

[45] D. Reynolds, X. Wang, and H.V. Poor, "Blind Adaptive Space-Time Multiuser Detection with Multiple Transmitter and Receiver Antennas," IEEE Tran. Signal Processing, vol. 50, no. 6, pp. 1261-1276, June 2002.

[46] K. G. Vardhe and D. Reynolds , "The performance of space-time coded cooperative diversity in a cellular uplink," in Proc. Asilomar Conf. Signals, Systems, Computers, Pacific Grove, CA, Nov 2005, accepted for presentation.

[47] K. G. Vardhe and D. Reynolds, "User cooperation in an asynchronous cellular uplink," Electronics Letters, submitted.

[48] B. Hassibi, B. Hochwald, "How much training is needed in multiple-antenna wireless links," IEEE Trans. Inform. Theory, vol. 49, no. 4, pp. 951-963, April 2003.

[49] D. Reynolds, "Statistical signal processing," Course Notes, WVU, Spring 2004.

[50] Erik Lindskog, Space-time processing and equalization for wireless communications, Ph.D. thesis, Uppsala University, Uppsala, Sweden, June 1999.

[51] S. Verdú, Multiuser Detection, Cambridge, UK: Cambridge University Press, 1998.

[52] N. Laneman, Cooperative diversity in wireless networks: algorithms and architectures, Ph.D. thesis, Massachusetts Institute Of Technology, Sept. 2002.

[53] R. Pickholtz, D. Schilling, L. Milstein, "Theory of spread-spectrum communications-A tutorial," IEEE Trans. Commun., vol. COM 30, no. 5, pp. 855-884, May 1982.

[54] T. Moon, W. Stirling, Mathematical Methods and Algorithms for Signal Processing, Prentice-Hall, Upper Saddle River, NJ, USA., 2000.

[55] A. S. Y. Poon, R. W. Brodersen, D. N. C. Tse, "Degrees of freedom in multiple-antenna channels: a signal space approach," IEEE Trans. Inform. Theory, vol. 51, no. 2, pp. 523-536, Feb. 2005. 


\section{A Code Division Multiple Access Technique (CDMA)}

A fundamental necessity of wireless communication system is that multiple users be able to simultaneously access and use the communication system. Multiple access systems allow finite number of users to share the limited radio spectrum. Allocation of resources like time slots or the frequency bands to every user is done in an orthogonal fashion. That is each user is provided with frequency or time slots that do not overlap with other users in the system.

FDMA (frequency division multiple access), TDMA (time division multiple access) and CDMA (code division multiple access) are the well-known multiple access techniques. FDMA assigns different frequency channels to different users while time slots are assigned to different users in a TDMA system. If the channel (frequency band in case of FDMA and time slot in case of TDMA) is not use, then it remains idle and can not be used by any other user to share capacity. TDMA systems require users to maintain time-synchronization. Also, the capacity of all three techniques is the same when perfectly synchronous AWGN channel is assumed. But there are significant differences in the capacities when more practical channels such as fading channels are taken into account [53] and capacity of CDMA is greater than that of TDMA and FDMA under practical situations. CDMA offers other advantages over FDMA and TDMA like universal frequency reuse, soft handoff, RAKE receiver reception to constructively combine multipath components and has been widely accepted in $3 \mathrm{G}$ standards, WCDMA and cdma2000.

CDMA is a spread spectrum technique in which signal is expanded in bandwidth with the help of a code which is independent of data. Synchronized reception and despreading operation with the same code is used to recover the original data. There are variations of CDMA technique, but the most commonly used class is DS-CDMA. (direct sequence -CDMA). Signals from different users now overlap in both time and frequency. There is no hard constraint on the number of users in a DS-CDMA system as in TDMA or FDMA system because all users in DS-CDMA use all of the system bandwidth all the time. DSCDMA system can continue to admit users until the interference between users causes the system performance to degrade to an unacceptable level. Thus, each user is assigned a individual signature waveform or a code and the receiver correlates the received signal with 
the generated signature waveforms to extract the information. The signature waveforms can be orthogonal or non-orthogonal. The number of users each with time duration $T$ and bandwidth $B$ that can be permitted with orthogonal signalling is given by [51]

$$
K=2 B T \text {. }
$$

The constraint on number of simultaneous users which is twice the time bandwidth product of signature waveforms can be relaxed if we allow non-orthogonal signature waveforms. Careful selection of signature waveforms should be done so that the cross correlation is low as compared with the individual signal energies. Also, non-orthogonality of signature codes can result due to asynchronous communication between transmitter and receiver. Throughout this thesis, we consider non-orthogonal signature codes for practical purposes. Specifically we use random codes in our simulations which gives a lower bound on the system performance.

The signature waveform of each user has the form

$$
s_{k}(t)=\sum_{n=0}^{N} c_{k}[n] \psi\left(t-n T_{c}\right)
$$

where $\boldsymbol{c}_{k}$ is the spreading code (or PN sequence) for the $k$-th user, $\mathrm{N}$ is the number of chips per bit and is also called as processing gain, $\psi(\cdot)$ is a unit energy baseband pulse (also called as chip waveform) which satisfies

$$
\int_{n T_{c}}^{(n+1) T_{c}} \psi(t) \psi\left(t-n T_{c}\right)=0, \quad n=1,2, \ldots
$$

Processing gain indicates the spreading factor. We have $T_{s}=N T_{c}$. Signal bandwidth in this case is $B=\frac{1}{T_{s}}$ and the bandwidth of the spreaded signal is $B_{\text {spread }}=\frac{1}{T_{c}}=N B$. As the original signal gets expanded by a factor of $N$, it is known as the bandwidth expansion factor or spreading factor or processing gain. Processing gain plays an important role in DS-CDMA communications. The number of synchronous or asynchronous signature codes grows with $N$, for a given maximum crosscorrelation level. Also, large values of $N$ improve the privacy of the system. 


\section{B Discrete Time Signal Model for Decode and Forward User Cooperation}

We derive the expression for the discrete time signal model for two-user decode and forward protocol. We consider asynchronous communication scenario. Table below shows symbols transmitted by two users in different time slots.

\begin{tabular}{|l|l|l|}
\hline Time Slot & User1 & User 2 \\
\hline \hline 0 & $b_{1}[1]$ & $b_{2}[1]$ \\
\hline 1 & $b_{1}[2]+b_{2}[1]$ & $b_{2}[2]+b_{1}[1]$ \\
\hline 2 & $b_{2}[2]$ & $b_{1}[2]$ \\
\hline
\end{tabular}

Table .1: Symbol transmissions from different users in different time slots for two-user DF sharing protocol.

From equation 4.7, we know that the continuous time signal received at the base station is

$$
\begin{aligned}
r(t)= & \alpha_{1} b_{1}[1] s_{1}\left(t-\tau_{1}\right)+\alpha_{2} b_{2}[1] s_{2}\left(t-\tau_{2}\right)+\alpha_{1} b_{1}[2] s_{1}\left(t-T_{s}-\tau_{1}\right)+ \\
& \alpha_{1} b_{2}[1] s_{2}\left(t-T_{s}-\tau_{1}\right)+\alpha_{2} b_{2}[2] s_{2}\left(t-T_{s}-\tau_{2}\right)+\alpha_{2} b_{1}[1] s_{1}\left(t-T_{s}-\tau_{2}\right)+ \\
& +\alpha_{1} b_{2}[2] s_{2}\left(t-2 T_{s}-\tau_{1}\right)+\alpha_{2} b_{1}[2] s_{1}\left(t-2 T_{s}-\tau_{2}\right)+n(t)
\end{aligned}
$$

where $\alpha_{1}\left(\alpha_{2}\right)$ is the complex Gaussian channel gain between user 1 (user 2) and the base station, $\tau_{1}$ and $\tau_{2}$ are the corresponding delays, and $n(t)$ is a white gaussian noise process. Also, $s_{k}(t)=\sum_{j=0}^{N-1} c_{k}[j] \psi\left(t-j T_{c}\right)$, where $c_{k}[j]$ is the $j$-th element of user $k$ 's spreading code and $\psi(t)$ is a unit-energy rectangular chip pulse waveform. At the receiver, as shown in Fig.1, we first perform matched filter detection with respect to pulse shape $\psi(t)$. Thus the matched filtered output during $i$-th time slot and $q^{\text {th }}$ chip interval is given by [45]

$$
y[i, q]=\int_{i T_{s}+q T_{c}}^{i T_{s}+(q+1) T_{c}} r(t) \psi\left(t-i T_{s}-q T_{c}\right) d t+n[i, q]
$$

where $n[i, q]$ is complex Gaussian noise, $T_{s}$ is the symbol duration, and $T_{c}=T_{s} / N$ is the chip period. When we deal with asynchronism $\left(\tau_{1}, \tau_{2} \in\left[0, T_{s}\right]\right)$, we match filter the continuous time received signal for one extra time slot. 
$y[i, q]=\left\{\begin{array}{l}b_{1}[1] \alpha_{1} \sum_{j=1}^{N} c_{1}[j] \int_{i T_{s}+q T_{c}}^{i T_{s}+(q+1) T_{c}} \psi\left(t-j T_{c}-\tau_{1}\right) \psi\left(t-i T_{s}-q T_{c}\right) d t+ \\ b_{2}[1] \alpha_{2} \sum_{j=1}^{N} c_{2}[j] \int_{i T_{s}+q T_{c}}^{i T_{s}+(q+1) T_{c}} \psi\left(t-j T_{c}-\tau_{2}\right) \psi\left(t-i T_{s}-q T_{c}\right) d t+ \\ b_{1}[2] \alpha_{1} \sum_{j=1}^{N} c_{1}[j] \int_{i T_{s}+q T_{c}}^{i T_{s}+(q+1) T_{c}} \psi\left(t-T_{s}-j T_{c}-\tau_{1}\right) \psi\left(t-i T_{s}-q T_{c}\right) d t+ \\ b_{2}[1] \alpha_{1} \sum_{j=1}^{N} c_{2}[j] \int_{i T_{s}+q T_{c}}^{i T_{s}+(q+1) T_{c}} \psi\left(t-T_{s}-j T_{c}-\tau_{1}\right) \psi\left(t-i T_{s}-q T_{c}\right) d t+ \\ b_{1}[1] \alpha_{2} \sum_{j=1}^{N} c_{1}[j] \int_{i T_{s}+q T_{c}}^{i T_{s}+(q+1) T_{c}} \psi\left(t-T_{s}-j T_{c}-\tau_{2}\right) \psi\left(t-i T_{s}-q T_{c}\right) d t+ \\ b_{2}[2] \alpha_{1} \sum_{j=1}^{N} c_{2}[j] \int_{i T_{s}+(q+1) T_{c}}^{i T_{s}+(q+1) T_{c}} \psi\left(t-T_{s}-j T_{c}-\tau_{2}\right) \psi\left(t-i T_{s}-q T_{c}\right) d t+ \\ b_{1}[2] \alpha_{2} \sum_{j=1}^{N} c_{1}[j] \int_{i T_{s}+q T_{c}}^{i T_{s}+(q+1) T_{c}} \psi\left(t-2 T_{s}-j T_{c}-\tau_{2}\right) \psi\left(t-i T_{s}-q T_{c}\right) d t \\ +n[i, q]\end{array}\right\}$

From the equality,

$$
\int_{i T_{s}+q T_{c}}^{i T_{s}+(q+1) T_{c}} k(t) \psi\left(t-i T_{s}-q T_{c}\right) d t=\int_{0}^{T_{c}} \psi(t) k\left(t+i T_{s}+q T_{c}\right) d t
$$

Equation B-5 can also be written as 


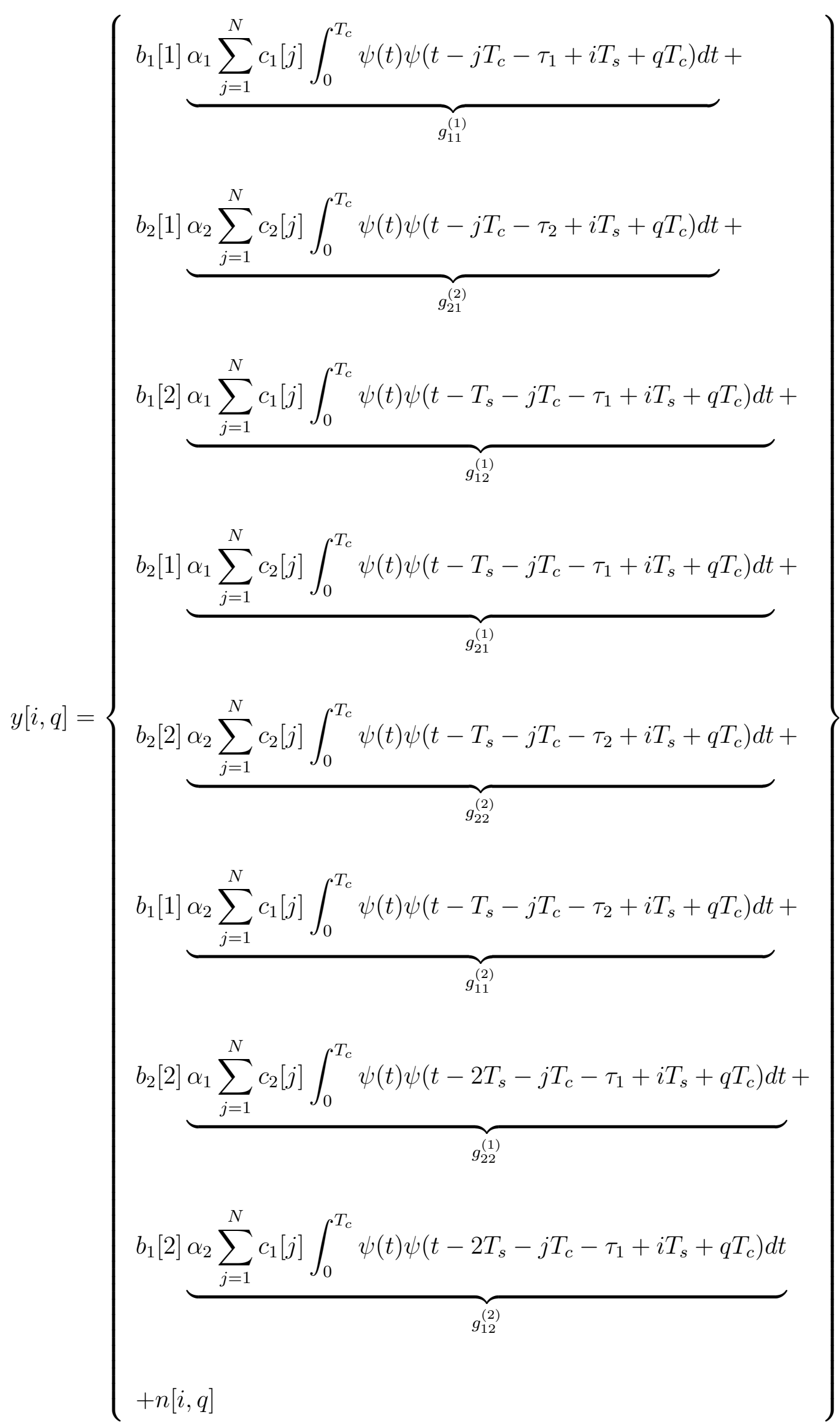


Let

$$
\begin{aligned}
& g_{11}^{(1)}[n, q]+g_{11}^{(2)}[n, q]=h_{11}[n, q] \\
& \begin{array}{cc}
\vdots & \vdots \\
g_{22}^{(1)}[n, q]+g_{22}^{(2)}[n, q]=h_{22}[n, q]
\end{array}
\end{aligned}
$$

Then

$$
y[i, q]=b_{1}[1] h_{11}[n, q]+b_{1}[2] h_{12}[n, q]+b_{2}[1] h_{21}[n, q]+b_{2}[2] h_{22}[n, q]+n[i, q]
$$

Define

$$
\tilde{\boldsymbol{y}}[n]=\left[\begin{array}{c}
y[n, 0] \\
\vdots \\
y[n, N-1]
\end{array}\right]_{N \times 1} \quad \boldsymbol{s}=\left[\begin{array}{c}
b_{1}[1] \\
b_{1}[2] \\
b_{2}[1] \\
b_{2}[2]
\end{array}\right]_{4 \times 1}
$$

Stacking all the match filtered outputs, we get

$$
\boldsymbol{y}=\boldsymbol{H} \boldsymbol{s}+\boldsymbol{n}
$$

where

$$
\boldsymbol{y}[n]=\left[\begin{array}{r}
\tilde{\boldsymbol{y}}[0] \\
\vdots \\
\tilde{\boldsymbol{y}}[3]
\end{array}\right]_{4 N \times 1}, \quad \boldsymbol{H}=\left[\begin{array}{cccc}
h_{11}[0,0] & h_{12}[0,0] & h_{21}[0,0] & h_{22}[0,0] \\
\vdots & \vdots & \vdots & \vdots \\
h_{11}[3, N] & h_{12}[3, N] & h_{21}[3, N] & h_{22}[3, N]
\end{array}\right]_{4 N \times 4}
$$

Linear MMSE filter can then be applied to $\boldsymbol{y}$.

Following the similar method, we can get the discrete time signal model for three-or-more user DF sharing schemes.

\section{Derivation for MMSE Filter Design}

Here we derive the expression for linear MMSE filter which was used in (4.14). We assume that the channel matrix is known at the receiver. 


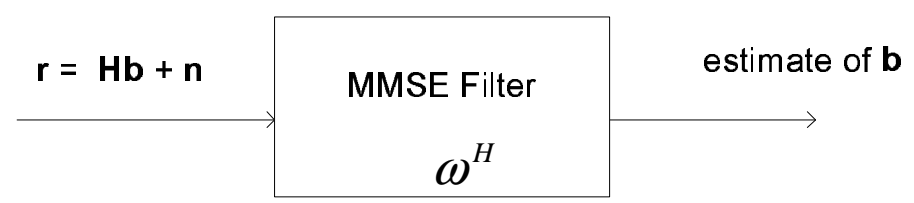

Figure .1: MMSE Receiver

Consider a received signal $\boldsymbol{r}=\boldsymbol{H} \boldsymbol{b}+\boldsymbol{n}$, where $\boldsymbol{H}$ is the effective channel matrix and is known at the receiver, $\boldsymbol{b}$ is the transmitted symbol vector and $\boldsymbol{n}$ contains samples of AWGN process. As shown in the above figure, minimum-mean-square-error (MMSE) filter operates on a discrete time received signal $\boldsymbol{r}$ to produce an estimate $\hat{\boldsymbol{b}}$ of the transmitted symbol vector $\boldsymbol{b}$. The cost function which MMSE filter tries to minimize is the expectation of meansquare error (MSE) between the filter inputs and outputs. Let $\boldsymbol{W}$ denotes the MMSE filter function. Then MMSE filter satisfies [15], [54]

$$
\boldsymbol{W}_{\text {opt }}=\arg \min _{\boldsymbol{W}} \mathrm{E}\left\{\left\|\boldsymbol{W}^{H} \boldsymbol{r}-\boldsymbol{b}\right\|\right\}
$$

Thus,

$$
\begin{aligned}
\mathrm{MSE} & =\mathrm{E}\left\{\left\|\boldsymbol{W}^{H} \boldsymbol{r}-\boldsymbol{b}\right\|\right\} \\
& =\mathrm{E}\left\{\left(\boldsymbol{W}^{H} \boldsymbol{r}-\boldsymbol{b}\right)\left(\boldsymbol{W}^{H} \boldsymbol{r}-\boldsymbol{b}\right)^{H}\right\} \\
& =\mathrm{E}\left\{\left(\boldsymbol{W}^{H} \boldsymbol{r}-\boldsymbol{b}\right)\left(\boldsymbol{r}^{H} \boldsymbol{W}-\boldsymbol{b}^{H}\right)\right\} \\
& =\mathrm{E}\left\{\boldsymbol{W}^{H} \boldsymbol{r} \boldsymbol{r}^{H} \boldsymbol{W}-\boldsymbol{W}^{H} \boldsymbol{r} \boldsymbol{b}^{H}-\boldsymbol{b} \boldsymbol{r}^{H} \boldsymbol{W}-\boldsymbol{b} \boldsymbol{b}^{H}\right\} .
\end{aligned}
$$

MSE is a quadratic function of $\boldsymbol{W}$ and can be minimized by taking the gradient w.r.t $\boldsymbol{W}^{H}$,

$$
\frac{\partial \mathrm{MSE}}{\partial \boldsymbol{W}^{H}}=\mathrm{E}\left\{\boldsymbol{r} \boldsymbol{r}^{H} \boldsymbol{W}-\boldsymbol{r} \boldsymbol{b}^{H}\right\}
$$

To find $\boldsymbol{W}$, we set MSE to zero. Thus we get

$$
\boldsymbol{W}=\mathrm{E}\left\{\boldsymbol{r} \boldsymbol{r}^{H}\right\}^{-1} \mathrm{E}\left\{\boldsymbol{r} \boldsymbol{b}^{H}\right\}
$$


The expectation in (C-16), is with respect to noise $\boldsymbol{n}$ and the symbol vector $\boldsymbol{b}$.

Consider,

$$
\begin{aligned}
\mathrm{E}\left\{\boldsymbol{r} \boldsymbol{r}^{H}\right\} & =\mathrm{E}\left\{(\boldsymbol{H} \boldsymbol{b}+\boldsymbol{n})(\boldsymbol{H} \boldsymbol{b}+\boldsymbol{n})^{H}\right\} \\
& =\mathrm{E}\left\{(\boldsymbol{H} \boldsymbol{b}+\boldsymbol{n})\left(\boldsymbol{b}^{H} \boldsymbol{H}^{H}+\boldsymbol{n}^{H}\right)\right\} \\
& =\boldsymbol{H E}\left\{\boldsymbol{b} \boldsymbol{b}^{H}\right\} \boldsymbol{H}^{H}+\boldsymbol{H} \mathrm{E}\left\{\boldsymbol{b} \boldsymbol{n}^{H}\right\}+\mathrm{E}\left\{\boldsymbol{n} \boldsymbol{b}^{H}\right\} \boldsymbol{H}^{H}+\mathrm{E}\left\{\boldsymbol{n} \boldsymbol{n}^{H}\right\} \\
& =\left(\boldsymbol{H} \boldsymbol{H}^{H}+\sigma^{2} \boldsymbol{I}\right)
\end{aligned}
$$

where we have assumed $\boldsymbol{b}$ and $\boldsymbol{n}$ are independent and the transmitted symbols are unit power, i.i.d random variables.

Now consider

$$
\begin{aligned}
\mathrm{E}\left\{\boldsymbol{r} \boldsymbol{b}^{H}\right\} & =\mathrm{E}\left\{(\boldsymbol{H} \boldsymbol{b}+\boldsymbol{n}) \boldsymbol{b}^{H}\right\} \\
& =\boldsymbol{H} \mathrm{E}\left\{\boldsymbol{b} \boldsymbol{b}^{H}\right\}+\mathrm{E}\left\{\boldsymbol{n} \boldsymbol{b}^{H}\right\} \\
& =\boldsymbol{H}
\end{aligned}
$$

Substituting (C-20) and (C-23) in (C-16), we get

$$
\boldsymbol{W}=\left(\boldsymbol{H} \boldsymbol{H}^{H}+\sigma^{2} \boldsymbol{I}\right)^{-1} \boldsymbol{H}
$$

Eqn. (C-24), gives the expression for MMSE filter. While deriving the above expression, we assumed that the channel is perfectly known at the receiver. But in practice, this may not be true. One solution is to replace expectation operators in (C-16) with time averaging. Or, we can estimate the channel using training sequence and then replace true $\boldsymbol{H}$ in (C-24) by the channel estimate. 


\section{Linear MMSE Channel Estimator}

In practice, channel is often estimated using blind techniques or with the help of training sequence. Here we derive the expression for linear MMSE channel estimator which was used in (5.3). We follow similar method as in Appendix C.

Suppose the discrete time signal model is represented by

$$
\boldsymbol{r}=\boldsymbol{H b}+\boldsymbol{n} .
$$

Channel is unknown at the receiver and needs to be estimated such that the cost function $\|\boldsymbol{r}-\boldsymbol{H} \boldsymbol{b}\|^{2}$ is minimized. This cost function is nothing but mean-square error.

$$
\begin{aligned}
\mathrm{MSE} & =\mathrm{E}\left\{(\boldsymbol{r}-\boldsymbol{H} \boldsymbol{b})(\boldsymbol{r}-\boldsymbol{H} \boldsymbol{b})^{H}\right\} \\
& =\mathrm{E}\left\{\boldsymbol{r} \boldsymbol{r}^{H}-\boldsymbol{r} \boldsymbol{b}^{H} \boldsymbol{H}^{H}-\boldsymbol{H} \boldsymbol{b} \boldsymbol{r}^{H}+\boldsymbol{H} \boldsymbol{b} \boldsymbol{b}^{H} \boldsymbol{H}^{H}\right\} .
\end{aligned}
$$

Since we want to estimate $\boldsymbol{H}$ that minimizes the MSE, we take the gradient of the above equation w.r.t. $\boldsymbol{H}^{H}$. Hence

$$
\frac{\partial \mathrm{MSE}}{\partial \boldsymbol{H}^{H}}=\mathrm{E}\left\{-\boldsymbol{r} \boldsymbol{b}^{H}+\boldsymbol{H} \boldsymbol{b} \boldsymbol{b}^{H}\right\}
$$

Equating gradient of MSE to zero, we get the expression for estimate of the effective channel matrix. It is given by

$$
\begin{aligned}
\boldsymbol{H} & =\mathrm{E}\left\{\boldsymbol{r} \boldsymbol{b}^{H}\right\} \mathrm{E}\left\{\boldsymbol{b} \boldsymbol{b}^{H}\right\}^{-1} \\
& =\boldsymbol{R}_{y s} \boldsymbol{R}_{s s}^{-1}
\end{aligned}
$$

We use this channel matrix to form a MMSE detector. In practice, expected values in (D-29) are replaced by time averaging operation. 


\section{E Information Theory Overview}

Information theoretic results are often used to determine the entropy and the achievable transmission rates for the system under consideration. In this section, we will introduce some of the basic terminologies used frequently in context of information theory while dealing with communication systems.

1. Degrees of Freedom in Communication Channels - In communication channels, the transmit and receive signals can be represented either in the time domain or the frequency domain. In time domain, signals are represented as waveforms and in the frequency domain, they are represented as spectra. Fourier transform gives the mapping between the time domain and the frequency domain representations. We know that the signal can not be band-limited as well as time-limited at the same time. But the signal waveform can be approximately time-limited to $\left[\begin{array}{ll}-T / 2 & T / 2\end{array}\right]$ and its spectrum can be frequency-limited to $[-W+W]$. The number of degrees of freedom $2 W T$ is then the dimension of the space which satisfies these two time and frequency constraints $[55]$.

2. Entropy - Entropy is the measure of information. It is nothing but a measure of uncertainty of a random variable.

Entropy $H(X)$ of a discrete random variable $X$ is by definition given by

$$
H(X)=-\sum_{x \in æ} p(x) \log p(x)
$$

3. Mutual Information - Mutual information is the amount of information one random variable contains about another.

If $X$ and $Y$ are two random variables, then the mutual information $I(X ; Y)$ is defined as

$$
\begin{aligned}
I(X ; Y) & =H(X)-H(X \mid Y) \\
& =H(Y)-H(Y \mid X)
\end{aligned}
$$


where $H(X \mid Y)$ and $H(Y \mid X)$ are the conditional entropies.

4. Channel Capacity - Channel capacity of a discrete memoryless channel is defined as

$$
C=\max _{p(x)} I(X ; Y)
$$

where the maximum is taken over all possible input distributions $p(x)$.

Another interpretation is that channel capacity is the highest rate in bits per channel use at which information can be sent over the channel with arbitrarily low probability of error.

5. Capacity of Gaussian Channel - Consider a simple Gaussian channel model,

$$
Y=X+Z
$$

where $Z \sim \mathcal{N}_{c}(0, N)$ and $Z$ is independent of $X$. Then the capacity of a Gaussian channel with power constraint $P$ and noise variance $N$ is

$$
C=\log \left(1+\frac{P}{N}\right) \text { bits/transmission }
$$

6. Capacity of a Rayleigh Fading Channel- Consider a Rayleigh fading channel where source transmits to ultimate destination via a direct link. Let the codeword length is very large. The received signal during one channel use of the channel can be written as

$$
y[n]=h x[n]+z[n]
$$

where $h$ is zero mean complex Gaussian random variable with variance $\sigma^{2}$, representing the channel gain and $z[n]$ is the complex Gaussian noise with variance $N_{0}$. Then, the average mutual information between input and output due to complex Gaussian inputs is $[52]$

$$
I=\log \left(1+\operatorname{SNR}|h|^{2}\right)
$$

$\mathrm{SNR}=\frac{P}{N_{0}}, P$ being the transmit power.

Note that the magnitude $|h|$ is Rayleigh distributed and $|h|^{2}$ is exponentially distributed with parameter $\frac{1}{\sigma^{2}}$. 


\section{Mutual Information with Code Combining Technique -}

Consider two sources transmitting to a destination terminal and having independent Gaussian codebooks. Let $\mathrm{SNR}_{1}$ and $\mathrm{SNR}_{2}$ be the signal to noise ratios of the respective source-destination links. Then the average mutual information between source and destination is

$$
I=\log \left(1+\mathrm{SNR}_{1}\right)+\log \left(1+\mathrm{SNR}_{2}\right) \quad \text { bits/transmission }
$$

\section{Mutual Information with Diversity Combining Technique -}

If now, sources employ the same Gaussian codebooks for transmission of information towards final destination, then average mutual information in this case is given by

$$
I=\log \left(1+\mathrm{SNR}_{1}+\mathrm{SNR}_{2}\right) \quad \text { bits/transmission }
$$

\section{F Scalar AWGN Channel Model with Decorrelating Multiuser Detection}

We consider a specific three-user cooperation scheme operating in an asynchronous uplink in the presence of multiple-access interference(MAI) and intersymbol interference (ISI). Considering symbol transmissions for three-user scheme as given in table (4.1) and matched filtering process as given in equation (B-7), we can write matched filtered output in $i^{\text {th }}$ time slot and $q^{\text {th }}$ chip interval as

$$
\begin{aligned}
y[i, q] & =b_{1}[1] \underbrace{\left(\alpha_{1} f_{11}^{(1)}[i, q]+\alpha_{2} f_{11}^{(2)}[i, q]+\alpha_{3} f_{11}^{(3)}[i, q]\right)}_{h_{11}[i, q]} \\
& +\quad \quad \vdots \quad \vdots \\
+ & b_{1}[4] \underbrace{\left(\alpha_{1} f_{14}^{(1)}[i, q]+\alpha_{2} f_{14}^{(2)}[i, q]+\alpha_{3} f_{14}^{(3)}[i, q]\right)}_{h_{14}[i, q]} \\
+ & \vdots \quad \vdots \\
+ & b_{3}[4] \underbrace{\left(\alpha_{1} f_{34}^{(1)}[i, q]+\alpha_{2} f_{34}^{(2)}[i, q]+\alpha_{3} f_{34}^{(3)}[i, q]\right)}_{h_{34}[i, q]}
\end{aligned}
$$


Stacking all the match filtered outputs, we get

$$
\boldsymbol{y}=\boldsymbol{H} s+\boldsymbol{n}
$$

where

$$
\boldsymbol{y}[n]=\left[\begin{array}{r}
\tilde{\boldsymbol{y}}[0] \\
\vdots \\
\tilde{\boldsymbol{y}}[6]
\end{array}\right]_{7 N \times 1}, \quad \boldsymbol{H}=\left[\begin{array}{cccc}
h_{11}[0,0] & \ldots & \ldots & h_{34}[0,0] \\
\vdots & \vdots & \vdots & \vdots \\
h_{11}[7, N] & \ldots & \ldots & h_{34}[7, N]
\end{array}\right]_{7 N \times 12}
$$

and

$$
\boldsymbol{s}=\left[\begin{array}{lll}
b_{1}[1] & \ldots & b_{3}[4]
\end{array}\right]^{T}
$$

We cam split $\boldsymbol{H}$ as

$$
\boldsymbol{H}=\tilde{\boldsymbol{H}} \boldsymbol{\alpha}
$$

where

$$
\begin{gathered}
\tilde{\boldsymbol{H}}=\left[\begin{array}{ccccc}
f_{11}^{(1)}[0,0] & f_{11}^{(2)}[0,0] & f_{11}^{(3)}[0,0] & \ldots & f_{34}^{(3)}[0,0] \\
\vdots & \vdots & \vdots & \vdots & \vdots \\
f_{11}^{(1)}[7, N-1] & f_{11}^{(2)}[7, N-1] & f_{11}^{(3)}[7, N-1] & \ldots & f_{34}^{(3)}[7, N-1]
\end{array}\right]_{7 N \times 36} \\
\boldsymbol{\alpha}=\left[\begin{array}{cccc}
\alpha_{1} & 0 & 0 & \ldots \\
\alpha_{2} & 0 & 0 & \ldots \\
\alpha_{3} & 0 & 0 & \ldots \\
0 & \alpha_{1} & 0 & \ldots \\
0 & \alpha_{2} & 0 & \ldots \\
\vdots & \alpha_{3} & 0 & \ldots \\
\vdots & 0 & \alpha_{1} & \ldots \\
\vdots & \vdots & \alpha_{2} & \ldots \\
\vdots & \vdots & \alpha_{3} & \ldots \\
\vdots & \vdots & \vdots & \ldots
\end{array}\right]_{36 \times 12}
\end{gathered}
$$

Thus we have

$$
\boldsymbol{y}=\tilde{\boldsymbol{H}} \boldsymbol{\alpha}+\boldsymbol{n}
$$

Decorrelating multiuser detector can now be applied as stated in (6.14) to obtain scalar AWGN channels which has representation quite similar to (E-37). 


\section{G Proof of Information Outage Probability expression for Multi-user Cooperation Scheme}

The proof we present here is akin to [37] with minor modifications due to specifics of our system model when applied to multi-user cooperation schemes. Any further details can be found in [37]. The mutual information between source and destination conditioned on decoding set for multi-user cooperation schemes is given by

$$
I_{\text {multi-user }}=\log \left(1+\frac{\mathrm{SNR}}{m\left[\boldsymbol{R}^{-1}\right]_{1,1}}\left|\mathrm{a}_{s, d}\right|^{2}\right)+\log \left(1+\frac{\mathrm{SNR}}{m} \sum_{i=r \in D(s)} \frac{\left|\mathrm{a}_{r, d}\right|^{2}}{\left[\boldsymbol{R}^{-1}\right]_{i, i}}\right)
$$

Thus it has the form,

$$
I_{\text {multi-user }}=\log \left(1+\frac{\mathrm{SNR}}{m} t_{m}\right)+\log \left(1+\frac{\mathrm{SNR}}{m} \sum_{k=1}^{m-1} t_{k}\right)
$$

In this case, $t_{k}$ is of the form $u_{k} / c_{k}, u_{k}$ being the exponential random variable with parameter $\lambda_{k}$ and $c_{k}=\left[\boldsymbol{R}^{-1}\right]_{k, k}$ being the constant.

Let $s_{m-1}=\sum_{k=1}^{m-1} t_{k}, t \triangleq\left(2^{R}-1\right), \epsilon=\left(2^{R}-1\right) /(\mathrm{SNR} / m)$ Then,

$$
\begin{aligned}
\operatorname{Pr}\left[I_{\text {multi-user }}<R \mid D(s)\right]= & \operatorname{Pr}\left[t_{m}+s_{m-1}+\frac{\mathrm{SNR}}{m} t_{m} s_{m-1}<\epsilon\right] \quad(\mathrm{G}-50) \\
= & \int_{0}^{\infty} \operatorname{Pr}\left[\left(t_{m}+s_{m-1}+\frac{\mathrm{SNR}}{m} t_{m} s_{m-1} \mid s_{m-1}\right)<\epsilon\right] \\
& \times p_{s_{m-1}}(s) d s \\
= & \int_{0}^{\epsilon} \operatorname{Pr}\left[t_{m}<\frac{\epsilon-s}{1+(\mathrm{SNR} / m) s}\right] p_{s_{m-1}}(s) d s
\end{aligned}
$$

By change of variables, $w=s / \epsilon$, we get

$$
\begin{aligned}
& =\epsilon \int_{0}^{1} \operatorname{Pr}\left[t_{m}<\frac{\epsilon(1-w)}{1+t w}\right] p_{s_{m-1}}(\epsilon w) d w \\
& =\epsilon \int_{0}^{1} \operatorname{Pr}\left[\frac{u_{m}}{c_{m}}<\frac{\epsilon(1-w)}{1+t w}\right] p_{s_{m-1}}(\epsilon w) d w \\
& =\epsilon \int_{0}^{1} \operatorname{Pr}\left[u_{m}<\frac{c_{m} \epsilon(1-w)}{1+t w}\right] p_{s_{m-1}}(\epsilon w) d w
\end{aligned}
$$

substituting CDF of $u_{m}$,

$$
\begin{aligned}
= & \epsilon \int_{0}^{1}\left[1-\exp \left(-\lambda_{m} \frac{c_{m} \epsilon(1-w)}{(1+t w)}\right)\right] \\
& \times p_{s_{m-1}}(\epsilon w) d w
\end{aligned}
$$


Now, we compute the limit. We make use of the following identities:

- Let $u$ be an exponential random variable with parameter $\lambda_{u}$. Then the $\operatorname{CDF} P_{u}(u)=$ $1-e^{-\lambda_{u} u}$ satisfies

$$
\lim _{\epsilon \longrightarrow 0} \frac{1}{\epsilon} P_{u}(\epsilon)=\lambda_{u}
$$

- Let $u_{k}, k=1,2, \ldots, m$ be positive, independent exponential random variables with

$$
\liminf _{\epsilon \longrightarrow 0} p_{u_{k}}(\epsilon u) \geq \lambda_{k}
$$

where $\lambda_{k}$ 's are the parameters.

and suppose we have $t_{k}=\frac{u_{k}}{c_{k}}, c_{k}$ 's being some constant. Then

$$
\liminf _{\epsilon \longrightarrow 0} p_{t_{k}}(\epsilon t) \geq c_{k} \lambda_{k}
$$

- Here we compute

$$
\lim _{\epsilon \longrightarrow 0} \frac{1}{\epsilon^{(m-1)}} \int_{0}^{1} p_{s_{m}}(\epsilon w) d w
$$

Exploiting Fatou's lemmma,

$$
\liminf _{\epsilon \longrightarrow 0} \frac{1}{\epsilon^{(m-1)}} \int_{0}^{1} p_{s_{m}}(\epsilon w) d w \geqslant \int_{0}^{1}\left\{\liminf _{\epsilon \longrightarrow 0} \frac{1}{\epsilon^{(m-1)}} p_{s_{m}}(\epsilon w)\right\} d w
$$

Now, $s_{m}=s_{m-1}+t_{m}$ and by independence of random variables, the PDF of $s_{m}$ is the convolution of the PDFs of $s_{m-1}$ and $t_{m}$. Since $t_{m}$ is positive, we have,

$$
\begin{aligned}
p_{s_{m}} & =\int_{0}^{s} p_{s_{m-1}}(s-r) p_{t_{m}}(r) d r \\
& =s \int_{0}^{1} p_{s_{m-1}}(s(1-y)) p_{t_{m}}(s y) d y .
\end{aligned}
$$

Denote

$$
A_{m}(w) \triangleq \liminf _{\epsilon \longrightarrow 0} \int_{0}^{1} \frac{1}{\epsilon^{(m-1)}} p_{s_{m}} d w
$$


Substituting (G-57) into (G-58)

$$
\begin{aligned}
A_{m}(w)= & \liminf _{\epsilon \longrightarrow 0} \int_{0}^{1} \frac{1}{\epsilon^{(m-1)}} p_{s_{m}} d w \\
\geqslant & w \int_{0}^{1}\left\{\liminf _{\epsilon \longrightarrow 0} \int_{0}^{1} \frac{1}{\epsilon^{(m-2)}} p_{s_{m-1}}(\epsilon w(1-y))\right\} \times \\
& \quad\left\{\liminf _{\epsilon \longrightarrow 0} p_{t_{m}}(\epsilon w y)\right\} d y d w \\
\geqslant & \lambda_{m} c_{m} w \int_{0}^{1} A_{(m-1)}(w(1-y)) d y
\end{aligned}
$$

The recursion gives

$$
A_{m}(w) \geqslant \frac{1}{(m-1) !} w^{(m-1)} \prod_{k=1}^{m} c_{k} \lambda_{k}
$$

Similarly, the upper bound lim sup can be found and knowing the fact that lim sup $\geqslant$ lim inf, we get the limit in equation (G-60) with equality.

Now to compute the limit in (G-51), we use (G-52) alongwith (G-60) and we obtain,

$$
\lim _{\epsilon \longrightarrow 0} \frac{1}{\epsilon^{m}} \operatorname{Pr}\left[I_{\text {multi-user }}<R \mid D(s)\right]=\frac{1}{(m-2) !} \prod_{k=1}^{m} \lambda_{k} \int_{0}^{1}\left[\frac{1-w}{1+t w}\right] w^{(m-2)} d w
$$

By large SNR approximation,

$$
\operatorname{Pr}\left[I_{\text {multi-user }}<R \mid D(s)\right] \sim\left[\frac{2^{R}-1}{\mathrm{SNR} / m}\right]^{m} \frac{1}{(m-2) !} \prod_{k=1}^{m} \lambda_{k} c_{k} \int_{0}^{1}\left[\frac{1-w}{1+\left(2^{R}-1\right) w}\right] w^{(m-2)}(\mathrm{G}-62)
$$

The above expression alongwith the expression for decoding set probability yields the final expression for the information outage probability. 